\title{
ABSTRACT
}

Title of Thesis: $\quad$ TIME REVERSED ELECTROMAGNETIC

WAVE PROPAGATION AS A NOVEL METHOD OF WIRELESS POWER TRANSFER

Frank Cangialosi, Anu Challa, Tim Furman, Tyler Grover, Patrick Healey, Ben Philip, Scott Roman, Andrew Simon, Alex Tabatabai, Liangcheng Tao

Thesis directed by: Dr. Steven Anlage

Department of Physics

We investigate the application of time-reversed electromagnetic wave propagation to transmit energy in a wireless power transmission system. "Time reversal" is a signal focusing method that exploits the time reversal invariance of the lossless wave equation to direct signals onto a single point inside a complex scattering environment. In this work, we explore the properties of time reversed microwave pulses in a lowloss ray-chaotic chamber. We measure the spatial profile of the collapsing wavefront around the target antenna, and demonstrate that time reversal can be used to transfer energy to a receiver in motion. We demonstrate how nonlinear elements can be controlled to selectively focus on one target out of a group. Finally, we discuss the design of a rectenna for use in a time reversal system. We explore the implication of these results, and how they may be applied in future technologies. 


\title{
TIME REVERSED ELECTROMAGNETIC WAVE PROPAGATION AS A NOVEL METHOD OF WIRELESS POWER TRANSFER
}

\author{
by \\ Team TESLA
Frank Cangialosi
Anu Challa
Tim Furman
Tyler Grover
Patrick Healey
Ben Philip
Scott Roman
Andrew Simon
Alex Tabatabai
Liangcheng Tao

Thesis submitted in partial fulfillment of the requirements of the Gemstone Program

University of Maryland, College Park

2016

Advisory Committee:

Dr. Steven Anlage, Mentor

Dr. Romel Del Rosario Gomez

Dr. Ray Sedwick

Dr. Biniyam Taddese

Dr. Vladimir Talanov 
(C) Copyright by

\section{Team TESLA}

Frank Cangialosi, Anu Challa, Tim Furman, Tyler Grover, Patrick Healey, Ben Philip, Scott Roman, Andrew Simon, Alex Tabatabai, Liangcheng Tao

2016 


\section{Acknowledgments}

Team TESLA would like to thank Nevenka Zdravkovska, the Gemstone Staff, and the graduate students of Dr. Anlage's lab for all of the help they have given us over the years.

In particular we would like to thank Dr. Anlage, Bo Xiao, and Rahul Gogna. Their guidance, patience, and support were critical to all of our successes. We learned so much from all of you. 


\section{Contents}

1 Introduction $\quad 1$

1.1 Introduction to Wireless Power . . . . . . . . . . . . . . . 1

1.2 Our Research ........................ 2

2 Literature Review $\quad 5$

2.1 Wireless Power Transfer Vocabulary . . . . . . . . . . . . . . 5

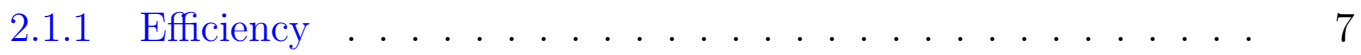

2.1.2 Maximum Power . . . . . . . . . . . . . . . 7

2.1.3 Active or Passive Transmission . . . . . . . . . . . . . . . 8

2.2 Health Concerns . . . . . . . . . . . . . . . . . . 8

2.3 Overview of WPT Technologies by Company . . . . . . . . . . . . . 9

2.3.1 Very Short Range . . . . . . . . . . . . . . . . . . . 9

2.3 .2 Medium Range . . . . . . . . . . . . . . . . . . . . . . . 10

2.3.3 Long Range . . . . . . . . . . . . . . . . . . . . . . . . . . . . 14

2.4 Time Reversal . . . . . . . . . . . . . . . . . . . . . 21

2.4.1 Conceptual Overview of Time Reversal . . . . . . . . . . . . . 21

2.4.2 History of Time Reversal Research . . . . . . . . . . . . . . . 24

3 Linear Time Reversal $\quad 28$

3.1 Purpose . . . . . . . . . . . . . . . . . . . . 28

3.2 Methodology for Linear Time Reversal . . . . . . . . . . . . . . . . . 29

3.3 Overlapping Reconstructions . . . . . . . . . . . . . . . . . . . 35

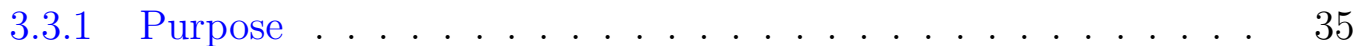

3.3.2 Methodology . . . . . . . . . . . . . . . 36

3.3.3 Results...................... . . . 40

3.4 Moving Reconstructions . . . . . . . . . . . . . . . . . 43

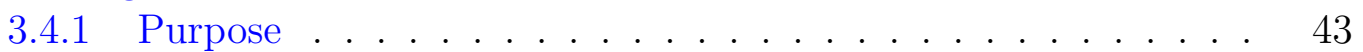

3.4.2 Spatial Profiling . . . . . . . . . . . . . . . . . 44

3.4.3 Reconstructing on a Moving Target . . . . . . . . . . . . . . . 48

3.5 Timing and Computational Complexity . . . . . . . . . . . . 51

3.6 Transfer Range . . . . . . . . . . . . . . . . . . 54

4 Nonlinear Time Reversal $\quad 58$

4.1 Experimental Difficulties . . . . . . . . . . . . . . . 61

4.1.1 Ferromagnetic Nanorods as a Nonlinear Beacon . . . . . . . . 61

4.1.2 Diodes as an Electric Nonlinear Beacon . . . . . . . . . . . . . 64

4.2 Purpose . . . . . . . . . . . . . . . . . 65

4.3 Methodology ........................ 66 
4.3 .1 Equipment. . . . . . . . . . . . . . . . . . 66

4.3.2 Time-Reversal and Nonlinear Sona Extraction . . . . . . . . . 67

4.3.3 Defining and Controlling the Nonlinear Element . . . . . . . 70

4.4 Results . . . . . . . . . . . . . . . . . . . . . . . . 72

4.4.1 Simultaneous Nonlinear Time Reversal . . . . . . . . . . . 72

4.4.2 Discussion of Simultaneous Nonlinear Reconstructions . . . . . 76

4.4.3 Simulation of Selective Collapse of NLTR . . . . . . . . . . . 77

4.4.4 Discussion of Selective Nonlinear Time Reversal . . . . . . . . 83

4.4.5 Simulation of Transmission Efficiency of NLTR Process . . . . 83

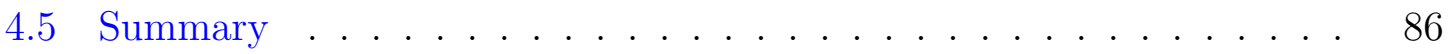

5 Development of a Super Rectenna $\quad 87$

5.1 Goals of Rectenna . . . . . . . . . . . . . . . . . . . . . . . 87

5.2 Diode Selection and Testing . . . . . . . . . . . . . . . 88

5.3 Antenna Design . . . . . . . . . . . . . . . . . . . . . . . . . . . . 90

5.4 Rectenna Testing . . . . . . . . . . . . . . . . . . . . . . . . . . 92

5.4 .1 DC Power Characterization . . . . . . . . . . . . . 92

5.4 .2 Harmonic Generation . . . . . . . . . . . . . . . . . . 96

5.5 Discussion . . . . . . . . . . . . . . . . . . . . . . . . . 99

6 Future Work 101

6.1 Vision of a WPT System using TR . . . . . . . . . . . . . . 101

6.2 Applying other techniques from TR to WPT . . . . . . . . . . 102

6.2 .1 Iterations . . . . . . . . . . . . . . . . . . . . . 103

6.2 .2 Multiple Transmitters . . . . . . . . . . . . . . . . 104

6.2 .3 Exponential Amplification . . . . . . . . . . . . . . . . 105

6.3 Novel Advancements for TR in a WPT context . . . . . . . . . . 106

6.3 .1 Sub Cavity . . . . . . . . . . . . . . . . 106

6.3 .2 Protection of Information _. . . . . . . . . . . . . . 109

6.3.3 TR WPT applied to Internet of Things Devices . . . . . . . . 111

7 Conclusion 114

7.1 Contributions . . . . . . . . . . . . . . . . . . 114

7.2 Evaluating Time Reversal as a Wireless Power Transfer Method ... 115

7.3 Final Words . . . . . . . . . . . . . . . . . . . . . . . . . 117

$\begin{array}{ll}\text { A Spatial Profile Fit Parameters } & 119\end{array}$

$\begin{array}{ll}\text { Glossary } & 120\end{array}$ 


\section{List of Tables}

2.1 Comparison of wireless technology companies and their products' capabilities ..................... 6

4.1 Computer specifications . . . . . . . . . . . . . 67

5.1 Datasheet for diode used in rectenna construction . . . . . . . . . . . 89

A.1 Spatial Profile Fit Parameters . . . . . . . . . . . . . . . . . . 119 


\section{List of Figures}

2-1 WiTricity schematic . . . . . . . . . . . . . . . 11

2-2 Phase Conjugation Example . . . . . . . . . . . . . . . . 15

$2-3$ Cota Process . . . . . . . . . . . . . . . . . . . 16

$2-4$ Cota Battery . . . . . . . . . . . . . . . . . 17

2-5 Wi-Charge: Typical laser . . . . . . . . . . . . . . . . . . . . . 19

2-6 Wi-Charge: Modified resonating cavity . . . . . . . . . . . . 19

2-7 Wi-Charge: Wi-Charge setup . . . . . . . . . . . . . . 19

2-8 Interrogation pulse, sona, and reconstruction from a typical TRM ex-

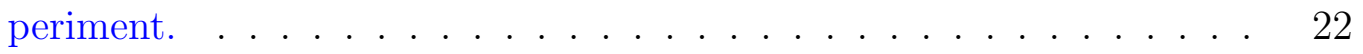

3-1 Experimental Setup . . . . . . . . . . . . . . . . . . . . 31

3-2 Experimental Setup: Gigabox Detail . . . . . . . . . . . . . . 32

3-3 Conceptual overview of linear time reversal . . . . . . . . . . . . . 33

3-4 Two-Port Linear Time Reversal Setup . . . . . . . . . . . . . 37

$3-5$ Overlapped sonas . . . . . . . . . . . . . . . . . 38

$3-6$ Overlapped reconstructions . . . . . . . . . . . . . . . 38

3-7 50 superimposed sonas . . . . . . . . . . . . . . . . . . . . . . 39

3-8 300 superimposed sonas . . . . . . . . . . . . . . . . . . . . 39

3-9 Max peak-to-peak voltage from overlapped reconstructions . . . . . 42

3-10 Total peak-to-peak voltage from overlapped reconstructions . . . . . . 42

3-11 MikroMove Translation Stage . . . . . . . . . . . . . . . . 45

3-12 Spatial profile of reconstruction at various frequencies . . . . . . 47

3-13 Fit of spatial profile . . . . . . . . . . . . . . . . . 47

3-14 Peak-to-peak voltage of moving reconstructions . . . . . . . . 50

3-15 Timing Analysis of Experimental Setup . . . . . . . . . . . . 53

4-1 Conceptual overview of nonlinear time reversal . . . . . . . . . . 59

4-2 Nanorods attached to antenna . . . . . . . . . . . . . . . . . . 62

4-3 Nanorod experimental setup . . . . . . . . . . . . . . . . . 62

4-4 Ferromagnetic nanorod experimental results . . . . . . . . . . 63

4-5 Example of inverted and non-inverted interrogation signals . . . . . 68

$4-6$ Demonstration of pulse inversion . . . . . . . . . . 70

4-7 CST diode circuit model . . . . . . . . . . . . . . . . . . . 73

4-8 I-V curves of diodes with different voltage knees . . . . . . . . . 73

$4-9$ The Cut Box Model . . . . . . . . . . . . . . . . . . . 74

4-10 $S_{11}$ spectrum of the Cut Box Model . . . . . . . . . . . . . 75

4-11 Simultaneous reconstructions on two diodes . . . . . . . . 77

4-12 Nonlinear response due to different diode characteristics . . . . . . . 79 
4-13 Selective reconstruction on a $V_{k}^{\text {low }}$ diode . . . . . . . . . . . . . . . 81

4-14 Selective reconstruction on a $V_{k}^{h i g h}$ diode . . . . . . . . . . . . . 82

4-15 Transfer efficiency for a two-diode simultaneous reconstruction . . . . 85

4-16 The Room Model . . . . . . . . . . . . . . . . 86

$5-1$ Rectenna design . . . . . . . . . . . . . . . . . . . . . . . . . . . . . . 91

$5-2$ Rectenna Rectification . . . . . . . . . . . . . . . . 94

5-3 Rectified DC power . . . . . . . . . . . . . . . . . . . . . 95

5-4 Measuring accepted AC power . . . . . . . . . . . . . . . . . . 97

5-5 Rectification efficiency . . . . . . . . . . . . . . . 98

5-6 Experimental setup for harmonic generation testing. . . . . . . . . . 98

6-1 Proposed Time Reversal System . . . . . . . . . . . . . . . . . . . . . 102

6-2 Proposed "Sub Cavity Design" . . . . . . . . . . . . . . . . . . . 109

6-3 Example of information loss on time reversal efficacy . . . . . . . . . 110

6-4 Proposed TR information protection system . . . . . . . . . . . . . . 112 


\section{Chapter 1: Introduction}

For over a century, we have become accustomed to simply plugging into a wall socket in order to power our devices. But what if the outlet could be taken out of the picture? What if these devices could operate beyond the confines of cord and cable? Although the concept of wireless electricity has existed since its proposal by Nikola Tesla in 1891, it was left largely uninvestigated throughout the twentieth century [1]. In the past decade, the proliferation of mobile devices and improved processing power have encouraged significant strides in the wireless power transfer (WPT) field. The age of wireless power has only just begun however, and there remain unexplored methods of transmitting this power to electronic devices.

\subsection{Introduction to Wireless Power}

The ability to wirelessly transport energy from one point to another has the potential to attract many unique investors and customers. Such a technology is convenient; it saves space by eliminating the need for unwieldy chargers or elusive wall outlets. Businesses that offer Wi-Fi connectivity may be interested in this technology as another service to both retain and grow their customer bases. A convenient method of recharging devices will discourage the use of non-reusable batteries and present environmental benefits. Residential consumers would certainly be interested in a 
convenient way to charge their cell phones, laptops, and other small devices. Automobile companies have demonstrated interest in wireless power technology, so users can charge their electric or hybrid cars more conveniently [2] [3].

The benefits of wireless power transfer (WPT) systems are myriad. Imagine an airport terminal, where individuals may wait for hours until their flight takes off. Many of these travelers will have a need to charge laptops, cellphones, and other electronic devices for both personal and work reasons. However, the layout of an airport terminal means that most people will be unable to reach a plug with their limited charging cords. Often, this creates a high amount of stress and unrest among travelers in the airport, particularly where individuals need their devices to get work done. This example can be extended anywhere there are more people who desire power for their devices, and the use of outlets is inconvenient. The college library is another paradigm in which hundreds of people are confined to a space where outlet access may be an issue. From domestic users to students and travelers, it is clear that millions of people will benefit from improved delivery of power.

\subsection{Our Research}

Team TESLA has examined a technique that has the potential to drastically improve the range of a WPT system and eliminate the issue of alignment. This technique, known as electromagnetic time reversal, has already been proven to be a successful method of information transfer [4,5], but its application to WPT has not yet been investigated. Time reversal (TR) is a method of locating the position of a 
receiver by utilizing the reflection and interference of waveforms in a complex environment. Because of this, we believe that TR may be an ideal method for WPT. Rooms and buildings contain complex surfaces that will allow TR to determine the location of the device's battery without knowing any information about the environment itself.

TESLA has investigated the feasibility of applying TR to WPT. In particular, we have considered the following questions:

- Can energy be transmitted consistently using TR?

- Can TR transmit energy to a moving target?

- Can TR selectively transmit energy to different targets?

It has already been proven that TR can transmit small packets of energy in the form of data signals [4]. However, the duration of the data signals was small compared to the spacing between them. As such, previous TR methods cannot be used to transmit energy as is. However, this technique can be modified to transmit packets of energy more rapidly. Our first experiments have shown that energy can be regularly transmitted using TR.

We have also demonstrated that TR WPT can effectively transmit energy to a moving target. Previous work in electromagnetic TR has exclusively used stationary targets. Time reversed systems are dependent on the reflections in a system, and even small changes in geometry (Such as a change in target position) result in a rapid deterioration of transfer quality. TESLA has demonstrated that a rapidly updating time reversal mirror (TRM) can compensate for the movement of the target. More 
importantly, we have characterized the behavior of such an updating system, allowing future TR WPT systems to be designed considering the result.

Finally, TESLA has explored the use of nonlinear time reversal (NLTR) to distinguish between targets in an environment with multiple possible targets. It was demonstrated by [4] that targets with nonlinear elements can be distinguished from linear targets. TESLA has used simulation software to demonstrate that this concept can be extended to distinguish between different nonlinear targets, a concept that may be necessary in the practical application of a TR WPT system. 


\section{Chapter 2: Literature Review}

Commercial wireless power transfer technologies have developed as a response to both the ubiquity of mobile devices and limitations in traditional wired power. These technologies differ in range, efficacy, and method, but suggest an overall attempt to shift away from traditional charging mechanisms. This literature review will focus on the distinct methods that make up the current state-of-the-art of wireless power. These methods and companies are not meant to be exhaustive, but should be recognized as a quick overview toward these liminal technologies. This review will additionally explore WPT technologies based on their applicable ranges.

At the conclusion of this overview of the state-of-the-art in WPT, we will discuss both the general operation of time reversal and the historical developments of that field. This will include the acoustical roots of TR by Claire Prada and Matthias Fink, later communication accomplishments by Steven Anlage, and TESLA's modern WPT application.

\subsection{Wireless Power Transfer Vocabulary}

Several groups have already made practical WPT technologies. In this paper Powermat, WiTricity, Wattup, uBeam, Cota, and Wicharge will be considered as the state-of-the-art WPT methods. The technologies these companies use in their prod- 


\begin{tabular}{|c|c|c|c|}
\hline Company & $\begin{array}{c}\text { Method of } \\
\text { Power Transfer }\end{array}$ & $\begin{array}{c}\text { Max Power } \\
\text { Delivered (W) }\end{array}$ & Approx. Range (ft.) \\
\hline Cota & Concentrated Microwaves & 1 & 30 \\
\hline Powermat & Inductive Coupling & 5 to 50 & Touching \\
\hline uBeam & Ultrasound & $\begin{array}{c}\text { Unknown } \\
\text { (minimum 1.5) }\end{array}$ & 3 to 13 \\
\hline WattUp & RF & 10 & 15 \\
\hline Wi-Charge & Laser & 10 & 30 \\
\hline WiTricity & $\begin{array}{c}\text { Resonant Inductive } \\
\text { Coupling }\end{array}$ & $\begin{array}{c}\text { Scalable, on the } \\
\text { order of } 1000\end{array}$ \\
\hline
\end{tabular}

Table 2.1: Comparison chart of wireless technology companies and their products' capabilities. The information in this table reflects publicly disclosed information at the time of writing.

ucts will be compared using several different metrics, defined below. The capabilities of these technologies under these metrics are summarized in Table 2.1, and discussed in detail in the following sections. These companies represent many of the major players in the current WPT industry that either currently have a product on the market, are in the process of commericalizing a product, or have plans to commercialize a product in the next few years.

As can be seen, the different WPT technologies vary wildly in both their methods of operation and their intended application. Comparing different WPT technologies is difficult because the measures of characterizing performance used in Table 2.1 are poorly defined. The field of WPT lacks one central governing body that defines such 
performance metrics. This, combined with the constant battle for market prominence leads to many crucial details being withheld due to Intellectual Property (IP) rights. Because of this, we will specify our own definitions of performance metrics important to this thesis.

\subsubsection{Efficiency}

Efficiency in particular is difficult to quantify, as there does not currently exist a standardized definition used by all parties. In the literature, efficiency of transfer has included:

- the amount of power delivered to the target compared to the amount of power drawn by the transmitter

- the transfer between antennas within a setup, with other losses (such as those due to rectifiers after transmission) being ignored

Here, the team defines efficiency as the amount of power delivered to the battery of the target device compared to the amount of electrical power input into the transmitter. We colloquially refer to this metric as "wall to load" efficiency.

The average efficiency of a corded phone chargers across eleven varieties of authentic, non-counterfeit chargers was found to be $72.4 \%[6]$.

\subsubsection{Maximum Power}

The maximum amount of power that can be transmitted to a target using a given technology. Typically, this value is cited at relatively short distances, much shorter 
than the advertised "maximum" range. It should be specifically noted that there is generally a tradeoff between maximum power and range that is unavoidable.

Maximum power limits the devices that can be powered by a given technology. While high power transmission is not important for all devices, a larger range of power improves the flexibility of the technology.

\subsubsection{Active or Passive Transmission}

Some technologies require the device being powered to actively participate in the powering process, usually by broadcasting a signal to the WPT transmitter. We call this active transmission. Others, like charging mats, are "always on" as long as the device to be powered is within range: from the device's point of view, this is passive transmission. This can be important because active charging requires the device to expend power while passive can accommodate a completely dead device.

\subsection{Health Concerns}

Another topic that needs to be considered is the health effects (if any) on living creatures in the range of a WPT technology. Electromagnetic radiation is known to have potentially harmful effects to biological tissue at sufficiently large power density levels [7]. The benchmark that is used to measure the applied power density that is transmitted to a person's skin is referred to as Specific Absorption Rating (SAR), which is a measure of the power deposited per unit mass of a material. In this case, the material is skin tissue and the SAR value is averaged over the entire body to 
arrive at a final value. In the US, the FCC legal limit on SAR is $1.6 \mathrm{~W} / \mathrm{kg}[8]$. As a reference, the iPhone 5 has a cited SAR value of $1.18 \mathrm{~W} / \mathrm{kg}$ [8]. It is generally accepted that cell phone use is safe. As long as our TR process does not exceed the 1.6 W/kg value, then we may assume it will be safe for human use and we do not need to be concerned with biological harm, as the FCC deems this a safe level of electromagnetic radiation.

\subsection{Overview of WPT Technologies by Company}

In order to classify the different companies, we have divided them by advertised range. For the purposes of this discussion, we note 3 ranges: (1) very short range (up to 1 foot), (2) medium range (max of 15 feet), and (3) long range (max of 30 feet). Now that we have defined a basic classification system for the current WPT technologies we may proceed to a more in-depth discussion of the companies themselves.

\subsubsection{Very Short Range}

\section{Powermat}

The most restrictive technology in terms of range is that of Powermat. Devices are charged by being placed on the charging mat. Harnessing inductive coupling, the same method used to charge electric toothbrushes, Powermat has virtually no charging range, as the device and the charger must be in full contact. The technology is capable of charging multiple devices, but the system requires compatibility devices for any that are not already Powermat-enabled off-the-shelf. 
As the charging mat is wired, its range is still restricted to the length of a power cord. If the device is not compliant with the Powermat standard, then it must be plugged into a compatibility device anyway. That said, the mat can deliver between $5 \mathrm{~W}$ and $50 \mathrm{~W}$, making it a very effective charging tool. Powermat compatible devices can be made without any external plugs, which could be desirable for applications such as waterproofing. Another interesting application has been the implementation of PowerMat devices inside furniture [9]. This allows tabletops to be transformed into charging surfaces.

\title{
2.3.2 Medium Range
}

\author{
WiTricity
}

Boston-based company WiTricity has expanded the previously discussed magnetic induction using a method they call highly resonant magnetic coupling (HRMC). Whereas traditional magnetic induction operates on the order of centimeters, HRMC is applicable at the meter scale. WiTricity reports $50 \%$ efficiency at two meters, followed by $10 \%$ efficiency at four meters $[10,11]$. HRMC has charged multiple devices without seemingly sacrificing overall efficiency [10]. The public demonstrations by WiTricity show that HRMC can transmit power ranging from milliwatts to kilowatts, significantly more than was practical with traditional induction [10].

HRMC uses magnetic induction to transmit energy between two coupled antennas. HRMC continually adjusts the coupling of transmitter and receiver antennas to keep both at a mutual resonant frequency. This technique, impedance matching, 


\section{Source Electronics}

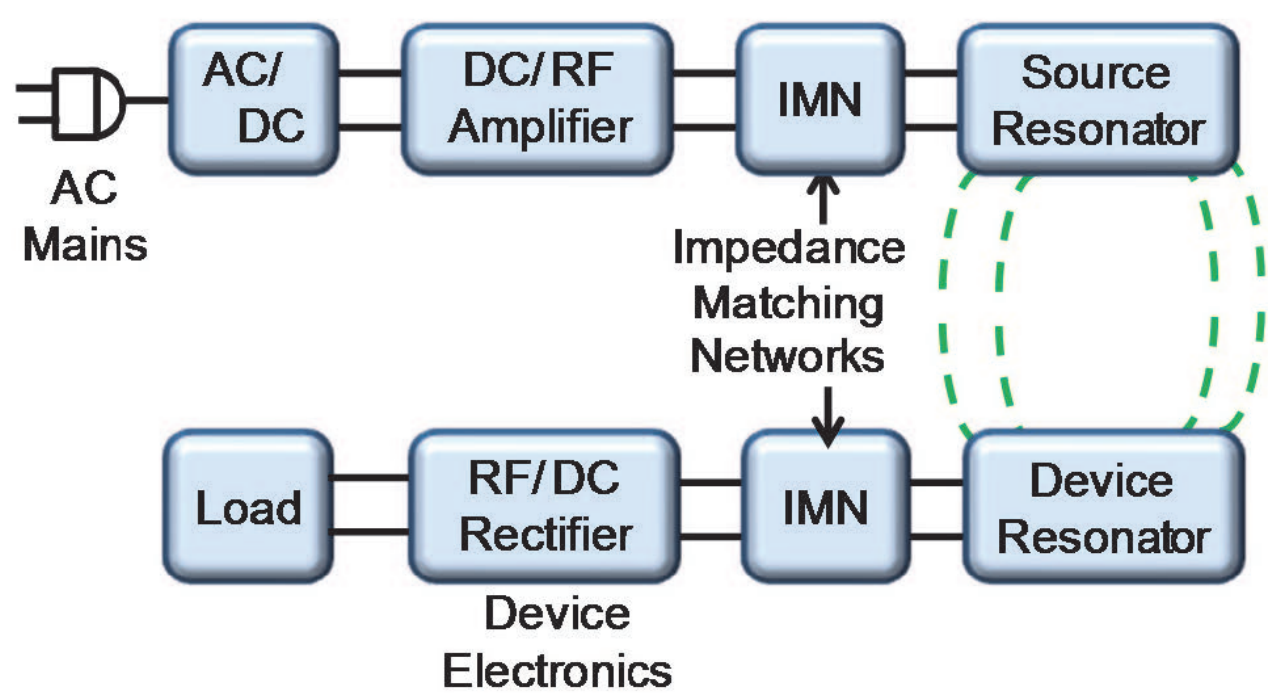

Figure 2-1: Schematic of WiTricity's HRMC System [10]

minimizes the amount of wave reflection inside the transmitter and receiver circuits, maximizing the power passed to the load component. This process requires a continuous proprietary feedback mechanism to optimize the receiver for the given distance from the transmitter.

WiTricity has demonstrated that the range of HRMC can be increased through the use of coupled relays. These relays can be coupled with the transmitter, allowing the effective range of the transmitter to increase with minimal additional loss [12].

\section{WattUp}

WattUp's central idea is to use Bluetooth to transfer information about the receiver's location relative to the transmitter, allowing the transmitter to directly focus energy on the receiver [13]. Once it has locked in a signal, it may boost the output power from $\mathrm{mW} R F$ signals to multiple $\mathrm{W} R \mathrm{RF}$ signals such that the receiver can 
charge. WattUp highlights two main features of its technology: (1) the range over which 1-4 W may be quickly and safely transmitted and (2) the relatively large number of devices that may be powered at once (currently 12, with 24 being available in the future) [13].

WattUp cites that they may deliver up to $4 \mathrm{~W}$ to 4 devices simultaneously within 0-5 feet of the transmitter, $2 \mathrm{~W}$ to 4 devices simultaneously within 5-10 feet, and $1 \mathrm{~W}$ within 10-15 feet [13]. This inverse relationship between delivered power and range is similar to the trend seen with WiTricity, as previously discussed. These numbers only represent the current model and demonstration version of the technology. WattUp claims that for ranges up to 5 feet, the device will charge as if it were plugged into a wall outlet, for 5-10 feet, the device will resemble USB port charging from a computer, and for 10-15 feet users can still power up at slower charge rates. Based on the aforementioned values provided by WattUp, the overall max range is about 15 feet with a max output power of about $10 \mathrm{~W}$, although these two conditions may not be met at the same time.

The technology itself uses two separate RF frequencies to perform wireless charging, representing both the Bluetooth communication $(2.4 \mathrm{GHz})$ and power transfer frequency $(5.7 \mathrm{GHz})[13]$. Both of these frequencies are allowed unlicensed frequency bands that are known to be used for wireless communication. We believe that WattUp chose to use separate frequencies in order to more easily differentiate the signals used for either targeting or power transfer. As the targeting algorithm is proprietary, we are unable to comment on their exact methodology for targeting the receiver. 
uBeam

The final primary medium range WPT technology, titled uBeam, uses an entirely unique method compared to other companies and methods, relying on ultrasonic waves rather than electromagnetic waves [14]. One can imagine their system as an extremely high power microphone and speaker combination that specifically sends the sound to a device location. Although uBeam is unique in its approach, it boasts comparable range and power levels when discussed next to other WPT companies.

The first difficulty of using sound waves as opposed to electromagnetic waves is the inherent dissipative nature of air as a medium. Due to this characteristic, uBeam uses output sound levels of $145-155 \mathrm{~dB}\left(316-3000 \frac{W}{m^{2}}\right)$, comparable to the sound produced by a jet engine or shotgun blast [15]. In order for this level of sound to be used safely, uBeam uses transmission frequencies of $45-75 \mathrm{kHz}$, as this is far above the audible range of both humans and animals. As an extra safety measure, uBeam cites that "if a person were to be exposed to the uBeam ultrasound source, $99.9 \%$ of the emitted ultrasound will bounce off the skin" [14]. These considerations have allowed uBeam to stay well within the FCC safety regulations. uBeam uses a phased array transmitter with thousands of antennas that result in a power range of 1-4 m. Although the tracking and targeting algorithm is proprietary in nature, uBeam claims the ability to maintain power transfer while the receiver is moving, although uBeam does not cite specific speeds. The technology requires few obstructions in order to work due to the nature of sound as a mechanical oscillation. For example, if a cell phone is in a user's pocket, then it will not be able to be powered, as the user's 
clothing will block or extremely dampen the ultrasonic waves.

As their product has not yet hit the market, numbers for uBeam's efficiency are not available. We do know that the power output from the transmitter is $145-155 \mathrm{~dB}$ [14], while a reasonable output at the receiver is on the order of 1-10 watts. Based on this, the technique seems unlikely to have a high wall-to-load efficiency.

\subsubsection{Long Range}

Cota

Cota $^{\mathrm{TM}}$ by Ossia uses phase conjugation of a continuous microwave signal in order to focus energy at a precise location. While this is a very similar concept to the time reversal process we will describe in the rest of this thesis, here phase conjugation is operated at a single frequency, compared to our time reversal process, which uses a wider bandwidth. The basic idea of phase conjugation is exemplified in Figure 2-2.

The current iteration of the Cota system at the time of writing consists of two components: a base station with a large array of 1,000 to 8,000 transmitting microantennas and a receiver device. The receiver device initiates the power transfer process by sending out a low power omni-directional beacon pulse 100 times per second, as shown in Figure 2-3(a), which propagates throughout the room, reflecting off walls, and eventually being recorded by the transmitter. The transmitter then calculates the "complex conjugate" of each wave recorded and transmits this mirror image of each wave, as shown in Figure 2-3(b), which ultimately converge at the receiver to deliver power [16]. Since the initial pulse is omni-directional, some waves will of 


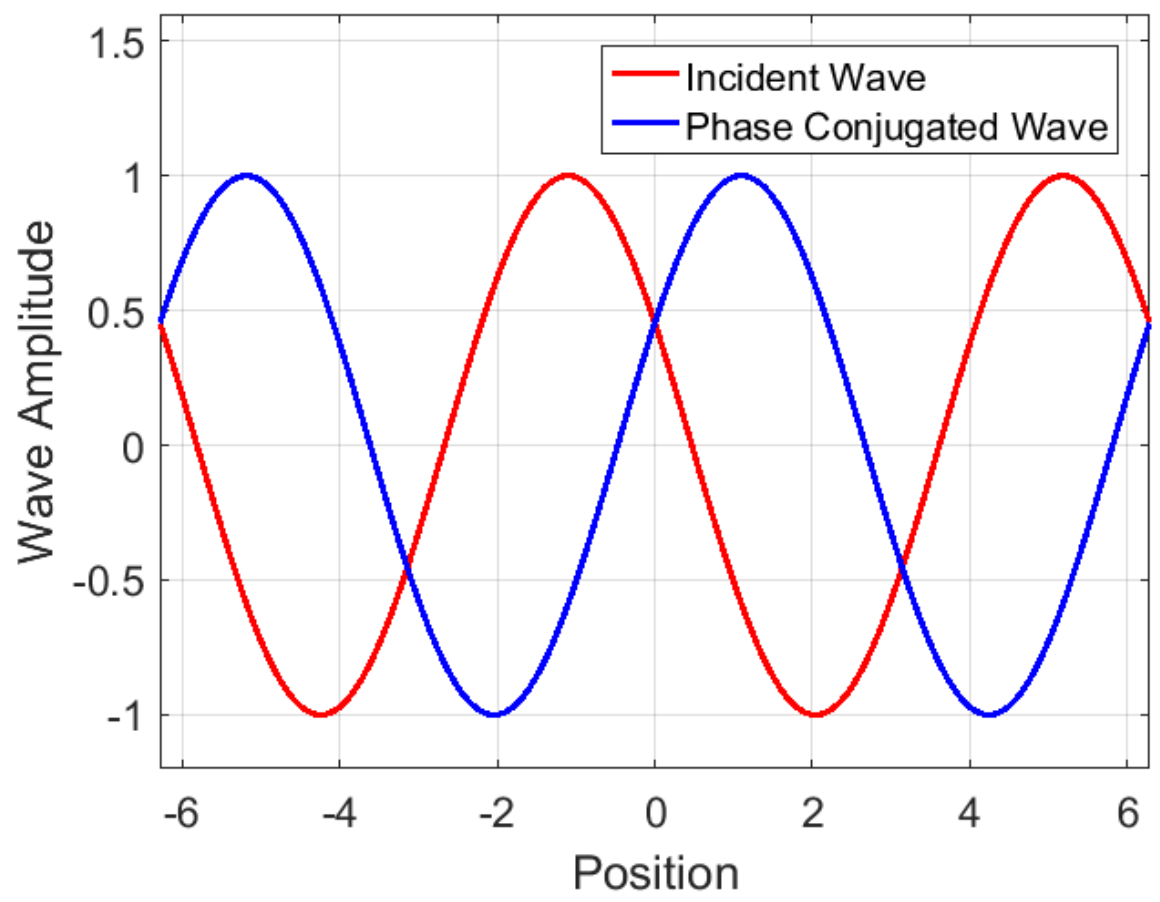

Figure 2-2: An initial incident wave received by a phase conjugate mirror and the corresponding phase conjugate reflected by the mirror. The phase conjugate wave is, unsurprisingly, a phase-shifted version (in this case offset by $\pi$ ) of the incident wave. 

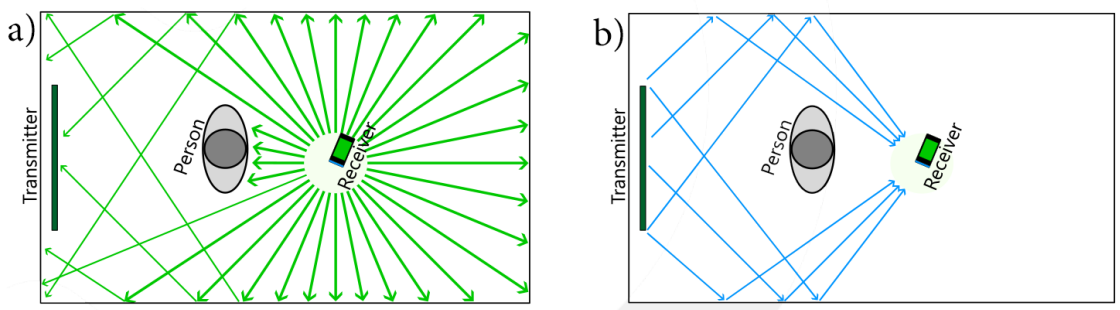

Figure 2-3: Description of the basic Cota power transfer protocol, which utilizes "multi-path" trajectories to avoid obstructions, as opposed to a "beam forming" power transfer system.

course end up hitting obstacles, such as people. However, the low power of this pulse makes them harmless, and the obstruction of the wave itself prevents this wave from reaching the transmitter, meaning that a higher power reflection will not make it back to the obstruction, and thus the technology naturally avoids obstacles. Since the position of the beacon is recalculated 100 times per second, they claim to be able to provide power to moving devices. The transmitter continually sweeps through a variety of frequencies in attempt to find the best $S_{21}$ parameter between the antenna and beacon. Even once it has settled on a frequency it continues this sweep and adjusts the frequency as the channel conditions change if necessary.

Cota's use of a single frequency simplifies the process because it means they only have to adjust the phase of the oscillator driving each antenna in the array. In addition, it allows them to work peacefully in a small pocket of the $2.4 \mathrm{GHz}$ ISM band, without interfering with the majority of the spectrum that is utilized by Wi-Fi devices. In addition, Zeine claims that lower frequencies have longer wavelengths that preclude the formation of "small" pockets of energy, while higher frequencies (such 


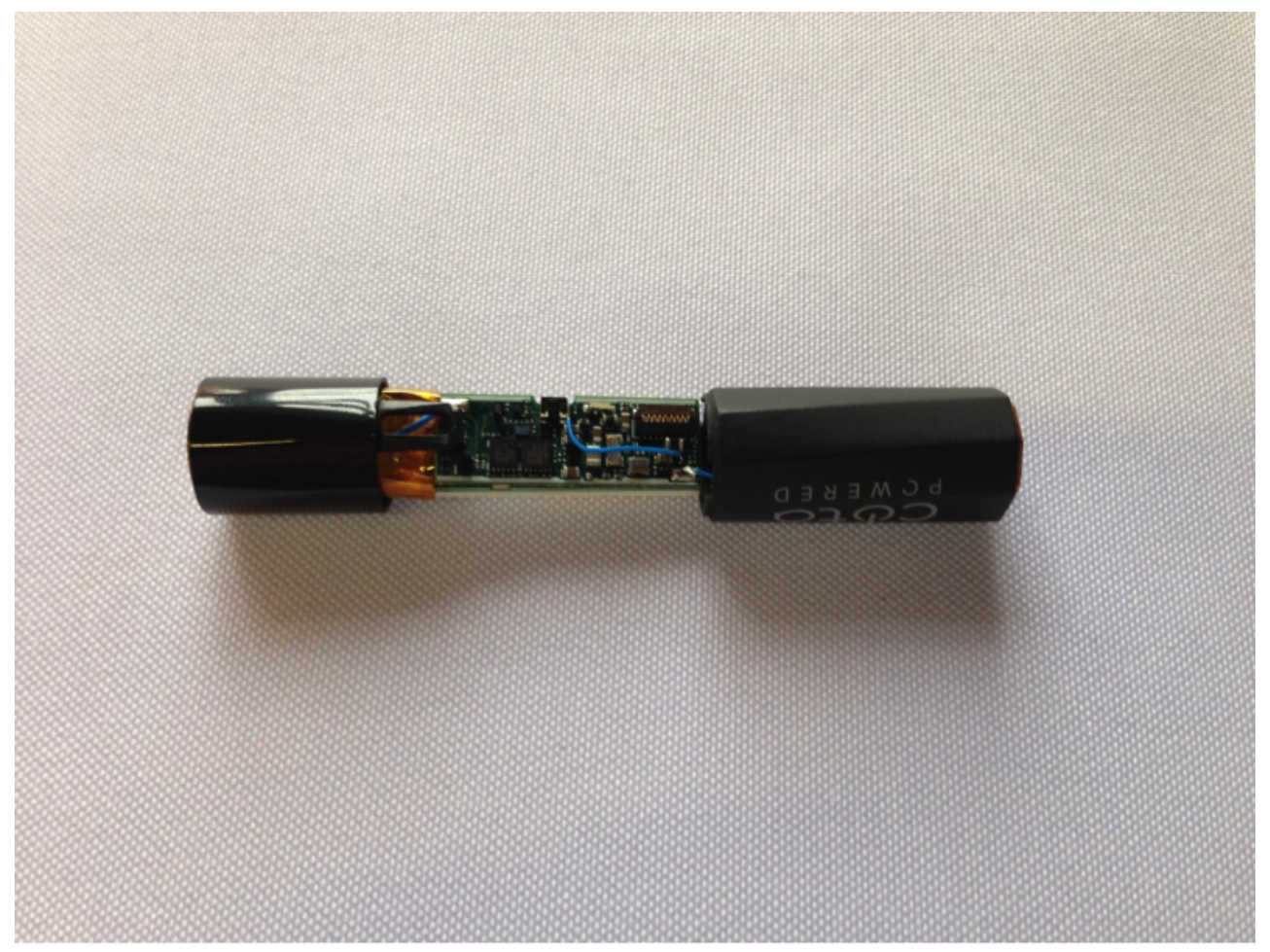

Figure 2-4: This photo of a Cota "AA Battery" was taken at the Ossia/Cota demonstration table at IEEE WPTC 2016. The technical representative stated that the battery constantly tops off power from the base station and stores this in a buffer until it is used by the device.

as $24 \mathrm{GHz}$ ) have much more stringent SAR limits set by the FCC.

Although many press releases about Cota claim that it can deiver up to 1 Watt of power at up to 10 meters, Zeine admitted that this was a goal, and not yet an achieved result (they are able to transmit up to 1 Watt of power, but only at unspecified "shorter" distances). They also were not able cite specific efficiency numbers, but admitted that they were "not high". The beacon currently broadcasts $24 \mathrm{dBm}$ at $100 \mathrm{~Hz}$, and the base station emits $5-10 \mathrm{~W}$ of power.

However, Cota has a grand vision for the improvement of these numbers and ultimately the incorporation of their technology into all of our mobile devices and the emerging set of "Internet of Things" devices. They already have created phone cases 
that can be attached to a typical mobile phone allowing existing devices to act as Cota receivers, though they ultimately envision that future devices will have Cota receivers built in. In addition, they have created "AA Batteries" (shown in Figure 2-4) that house the entire Cota receiver system, which both exemplifies how small the receiving circuit can be, and also allows Cota to retrofit the vast array of devices that currently rely on batteries without any modifications to the device. Zeine claims that they are currently working with paint and carpet manufacturers to make their products more conductive, which would provide better reflections for power transmission.

It is worth noting that, at the time of writing, Ossia has not yet released any peerreviewed publications or white papers detailing the Cota technology. The information above was compiled from Ossia's "Wireless power transfer system patents" [17] and conversations with Ossia's CEO, Hatem Zeine, at the 2016 IEEE Wireless Power Transfer Conference in Aveiro, Portugal.

\section{Wi-Charge}

Also capable of a $30 \mathrm{ft}$ operating radius, Wi-Charge uses a combination of a laser cavity and a photovoltaic cell to create robust power transfer systems [18]. To understand the system, it is necessary to discuss how a laser works. Consider Figure 25, which depicts two mirrors and an amplifier between them. Simply put, one photon, an energetic light particle, enters the amplifier and two come out. When the photons hit the mirror, they bounce back through the amplifier in the opposite direction and two photons become four. This process repeats with each ricochet against a mirror, and the energy amplifies within the system. This process of recirculating light in a 

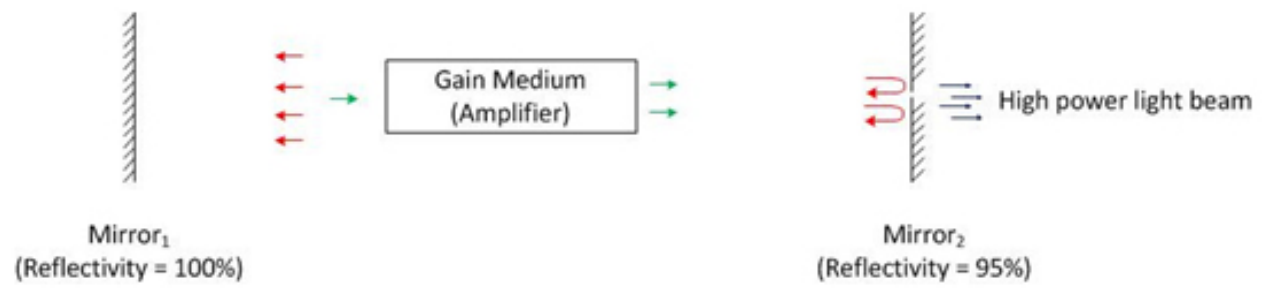

Figure 2-5: The resonating chamber of a typical laser. The gain medium allows for stimualted emission of photons. The 95\% reflective mirror partially transmits light, which is observed as the output of the laser [18].

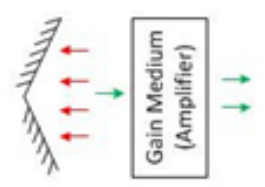

Retro Reflector Mirror (Reflectivity $=100 \%$ )

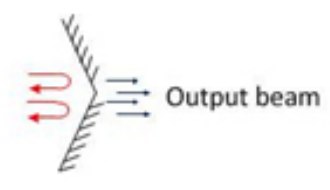

Retro Reflector Mirror 2 (Reflectivity $=95 \%$ )

Figure 2-6: A modification of the traditional laser resonating cavity to allow for collimation and coherency of the stimulated photons [18].

\section{Transmitter}

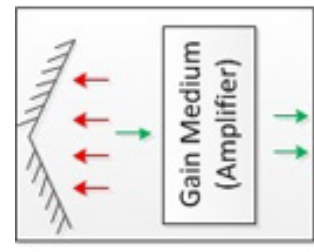

Link (free space)

Safe \& Hazard Free!
Receiver

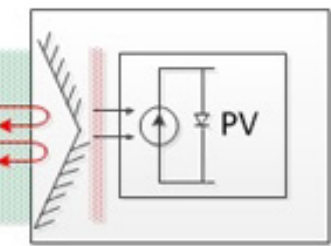

Figure 2-7: The setup of the Wi-Charge system. The fully reflective mirror is on the transmitter, while the $95 \%$ reflective mirror is located within the receiver device. Behind the receiver's mirror is a photovoltaic cell to convert the light back into an electrical signal to power a battery [18]. 
positive feedback loop with an amplifier creates a resonator.

When that resonator has one mirror that is very slightly transparent, as in Figure 2-5, then a focused, high powered beam is generated. This is a laser beam.

Any particles that do not travel along the axis between the two mirrors will hit the mirror at an odd angle and bounce out of the resonator. This is why only the photons that are traveling in the direction of the axis between the two mirrors will continue to amplify.

Wi-Charge took this traditional definition of a laser, and made a few clever modifications to better suit their purposes, shown in Figure 2-6 [18].

First, they kept the components-two mirrors with an amplifier between them-but modified their arrangement. Second, they made the mirrors retro-reflectors which, unlike regular mirrors, reflect light back to their sources. The result is that the two mirrors spontaneously form a resonator when placed within each other's line-of-sight, although the resonator is stalled immediately when the line-of-sight is broken. This last property may actually be desireable in a consumer setting, as it helps to prevent energy from accumulating anywhere but the intended receiver.

In Wi-Charge's setup, one mirror and the amplifier are grouped together as the transmitter, and a second mirror and a photovoltaic cell are grouped together as the receiver. This is shown in Figure 2-7. By placing the photovoltaic cell directly after the laser output location, the Wi-Charge system effectively converts the optical signal back to an electrical signal that can be used to charge a battery.

Wi-Charge is a long range wireless power solution that can deliver up to $10 \mathrm{~W}$ of power [18]. It can latch onto targets almost instantaneously and cease beaming 
equally quickly, intrinsically. It can also power multiple devices. However, the system does require line-of-sight.

\subsection{Time Reversal}

\subsubsection{Conceptual Overview of Time Reversal}

The technique known as time reversal (TR) uses properties of waves to focus a signal onto the point of origin of some other, previous signal. To do this, the technique exploits two properties of electromagnetism: reciprosity and time reversal symmetry. Reciprocity is a class of theorems in electromagnetism that say if the locations of source and measurement are exchanged, the measurement is unchanged. Time Reversal Symmetry is the property that a physical parameter is invariant under a time reversal operation $(T: t \rightarrow-t)$. Using these properties, TR can locate objects without prior knowledge of their position or their surrounding geometry.

A system that performs TR is called a "time reversal mirror" (TRM), sometimes simply shortened to "mirror." A TRM consists of one or more transmitters to introduce waves into a chamber and one or more receivers to record the echoes from it. The transmitters and receivers may (but do not have to be) the same device. TR requires an at least partially echoic chamber that allows waves to reflect off of its interior geometry and return to the transmitters. Here we refer to such a chamber as "reverberant".

A TRM works by broadcasting a waveform into the reverberant chamber and recording the resultant echo. This echo will consist of many time-shifted overlays 


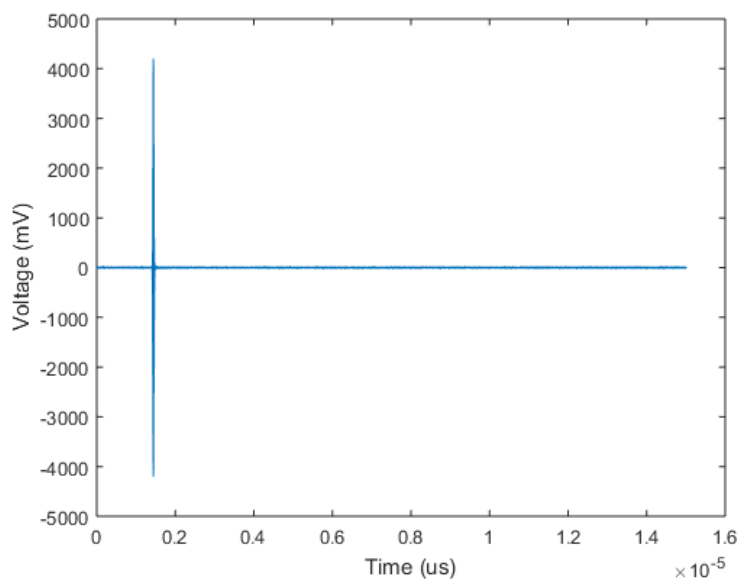

(a) Example interrogation pulse

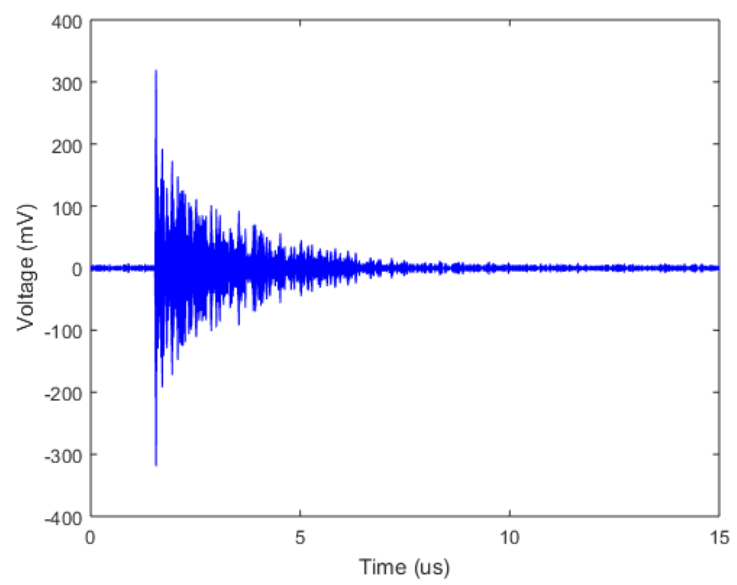

(b) Example sona

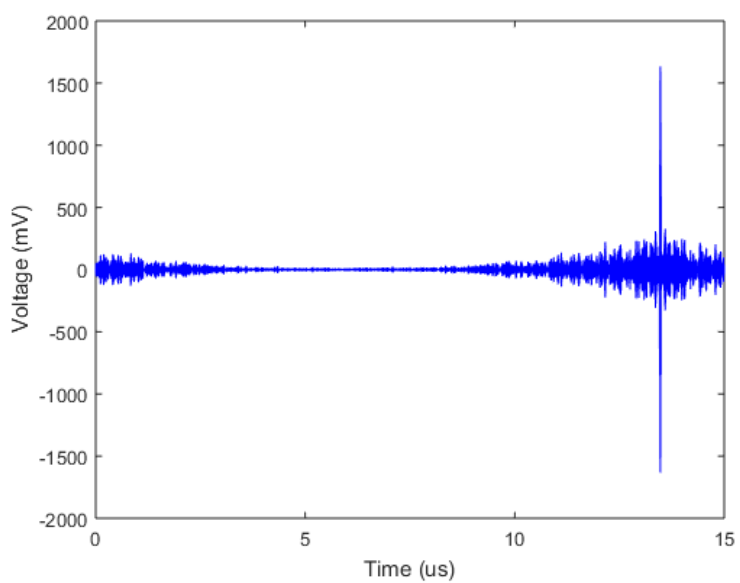

(c) Example reconstruction

Figure 2-8: Interrogation pulse, sona, and reconstruction from a typical TRM experiment. 
of the original waveform. We refer to the initial signal as an "interrogation pulse," and the echo as a "sona" in this thesis. An example interrogation pulse is shown in Figure 2-8a. An example sona is shown in Figure 2-8b. The sona is time reversed and rebroadcast, which will cause the waves to trace their paths in reverse and return to their source in a semi-coherent "reconstruction" of the original broadcast. An example reconstruction is shown in Figure 2-8c: in this case, the original signal was a short pulse in the same shape as the large vertical spike near the right of the figure. Since it involves waves from many scattered paths all suddenly arriving at the same point, a reconstruction is also known as "collapse."

In an ideal theoretical scenario, if we could capture all path information in the sona, a perfect reconstruction would be a recreation of the initial interrogation pulse. In reality, since we are unable to perfectly capture the sona due to noise and loss, a resulting reconstruction will bear additional features in the time domain not present in the interrogation pulse (as can be seen in our example reconstruction). These features about the center peak of the reconstruction are referred to here as "side lobes," artifacts of the imperfections in the sona. As the sona is recorded, the reverberations from the interrogation pulse disperse, and eventually fall beneath the noise floor for measurement. When time reversed, the lost information produces the side lobes seen above. Side lobes represent an incomplete control of the reconstruction, and are to be avoided. Thus, quantitatively, one can define the quality of a reconstruction as the ratio of the peak to the side lobes or to the root-mean-square noise floor.

$\mathrm{TR}$ is relatively versatile. If the technique is iterated exactly as described, the broadcasts will collapse more and more exactly on the strongest scatterer in the 
chamber [19]. Alternatively, prior work in acoustics has explored super-resolution as a method of maximizing the quality of the reconstruction [20-22]. Many other methods can be used to select targets more discriminately, and the generic TRM itself can be modified as well - for example, by recording directly from a target position, it is possible to create a secure channel between that point and the transmitter [4].

\subsubsection{History of Time Reversal Research}

TR is a proven technique in signal processing, with applications in acoustics as well as electromagnetics. Though its publicity is limited, there is a wealth of available literature regarding TR in certain specialized areas. Here we briefly describe the development and historical applications of the technique to illustrate where TESLA's project will expand this literature.

Time reversal as a technique was first developed in the 1990s. Some of the earliest and most influential work was conducted through teams led by Mathias Fink and Claire Prada of the University of Paris. These researchers used the technique to focus sound waves on "scatterers," objects that reflect the pulses [23]. An array of transducers would fire a sonic pulse into some propagation medium and listen for the echo. The recording of that echo was reversed in the time domain and transmitted back into the medium. They repeated (iterated) this process, causing the acoustic signature of the strongest scatterer to appear more prominently each time. In this way, the team was able to iteratively focus on the scatterers without needing prior knowledge of their location. Prada and her team submitted this DORT (French acronym, English: Decomposition of the Time Reversal Operator) method as a process for 
finding cracks or faults in structural members [23]. More importantly, Prada et al. went on to demonstrate that the method could always resolve the brightest scatterer if given enough iterations, that it worked better in a heterogeneous medium than a homogenous one, and that it was both experimentally and mathematically possible to resolve multiple targets at once [24]. These discoveries generated significant interest in a subset of the acoustics research community.

Others in the field of acoustics went on to refine the DORT method as an imaging technique, and as the field gathered attention, further explored formalizing the problem in general. An excellent example of the latter is the theoretical work by D. H. Chamber in his 2007 examination of TR for target detection and characterization [25]. In 2010, Nguyen and Gan developed a way to extract much more information from an anisotropic (directionally distinct) scatterer, including its rough shape, density, and radius. In doing so, they developed a faster mathematical approach to locating their scatterers that relied on several good approximations instead of one exhaustive computation [26]. Also in 2010, Barbieri and Meo made a large contribution to the field by bringing together the DORT method, which works in linear environments, and another similar method for working in nonlinear environments. This allowed them to resolve and distinguish between linear scatterers such as holes and nonlinear scatterers such as cracks [27].

Imaging is not the only application for a focusing method, however, and others adapted the existing body of TR research to new problems. The reciprocity of the wave function that Fink and Prada relied on to develop the technique holds for all waves it can be used to model. This means that the time-reversal operation works 
much the same way with electromagnetic waves as it does with sound waves [25]. This was explored as early as 1999, but was largely concerned with the same imaging problems occupying those in acoustics until at least 2007 [25]. However, that gradually began to change. In 2011, a team including graduate students from the University of Maryland posited that time reversal was an ideal mechanism for wireless communication [28]. In the same way that a sound wave could be made to collapse on a scatterer, they showed that an information packet could be made to collapse on a receiver. The team submitted this as an eco-friendly communication method because information transfer could be accomplished using less energy [28]. Later, this same property was examined for its security benefits instead of its environmental ones. In February of 2013, a team of researchers at the University of Maryland, including Matthew Frazier and Steven Anlage, published a study discussing TR as a method to selectively send information in a chaotic wave environment $[4,29]$. The team was able to create an exclusive communication link to a certain object, without needing to know its location and without interfering with nearby objects. In their experiment, they were able to transmit data (in the form of images) exclusively to a desired port, while the other port received only garbled noise.

Beyond its practical applications, in the process, Frazier and his team used nonlinear elements to extend TR in new and exciting ways. Recall that in traditional TR many iterations are required to pinpoint the target. The addition of a significantly nonlinear element greatly simplifies the pinpointing process. When a wave strikes the element, harmonic frequencies are produced at integer multiples of the original frequency. These harmonics can be quickly located in the echo's frequency domain and 
filtered in order to select them exclusively. The important distinction is that since the harmonics originated directly from the target antenna, all subsequent broadcasts of the TR signal will collapse on the antenna without the need for iteration [4]. Frazier and the others put forth several exciting directions to pursue with this concept: the secure communication channels mentioned above, hyperthermic treatment of tumors, and a long range WPT system that eschews traditional high power beams. It is this last area that TESLA intends to explore. 


\section{Chapter 3: Linear Time Reversal}

\subsection{Purpose}

In investigating the applicability of time reversal to a wireless power transfer system, there are several properties that we would like to characterize further. While transferring a meaningful amount of energy to a receiver is the primary goal, there are other characteristics to consider as well. We are interested in being able to transmit qdequate energy to a receiver in a complex environment, which may have an obstructed line of sight. We are also interested in transferring energy to a receiver in motion.

Of first importance is the transferring meaningful amounts of energy. Here, "meaningful" is a relative term; depending on the application and what is being powered with the WPT scheme, the demand for power will vary. The amount of energy required to charge an electric car (on the order of $3 \mathrm{~kW}$ ) will be vastly larger than the amount of energy required to charge a mobile phone (on the order of $10 \mathrm{~W}$ ), for example. [30] Our target application is the charging of cell phones, so it is necessary to investigate how much power can be transmitted safely using TR. For this specific WPT technique, this translates into the size and frequency of pulses that can be sent to the receiver.

We also would like to characterize the extent to which TR can be used to charge 
a receiver in motion. Motion of either the receiver or transmitter is a limitation of many WPT technologies; we ask if TR is also subject to this limitation. Time reversal research has focused primarily on the technique's use in stationary environments with stationary transceivers. Some research has shown that TR can be used as a motion detector, relying on the destruction of TR fidelity correlated with a change in the environment. [29] However, we would like to investigate the effect of small receiver position changes on the TR process.

Understanding these phenomena allows us to make a judgment on whether TR could be used as an effective WPT technique. If TR can transmit large amounts of energy to a receiver, it fulfills the primary goal of a WPT scheme and merits further examination. Consideration of the more advanced goal of sending energy to a receiver in motion is more detailed. This ability is a gap in the current market, and is not prominent in current WPT technologies. Having identified this shortcoming, TR can fulfill a need that is not currently being met if it is able to target a receiver in motion.

\subsection{Methodology for Linear Time Reversal}

We conducted physical experiments to answer the following questions:

- What modifications will allow TR send a meaningful amount of power to a receiver?

- How can TR be used to target receivers in motion?

Both of these proposals require similar methodologies for investigation. As such, the experiments we conducted shared much of the same equipment and procedure. Here 
we will detail the process that we used for establishing a linear time reversal mirror between two ports.

These experiments take place within an enclosed, reflective cavity called a "Gigabox" for ease of reference: an aluminum box with a metallic foil scattering paddle to make the ray trajectories more ergodic. The resulting ray chaos ensures that a propagating waveform will eventually reach every point in the environment. This is essential for the proper function of a time reversal mirror. Up to five identical monopole antennas can inject and extract electromagnetic signals from different ports in the enclosure, but for these experiments only two were used. One antenna was held stationary, while the other was mounted on a scanning window that could accommodate motion of up to $70 \mathrm{~mm}$ in one dimension. Ports on opposing sides of the Gigabox were selected to avoid confounding short orbit effects which result from an insufficient mode density between the injecting and receiving ports.

Our time reversal scheme consists of several pieces of microwave processing equipment and a desktop workstation. Interrogation pulses and time-reversed sona signals are created and broadcast using a Tektronix AWG7052 arbitrary waveform generator feeding an Agilent E8267D Vector PSG microwave source. A digital storage oscilloscope (DSO, Agilent DS091304A) is used to record waveforms of interest. MATLAB Version 2015a is used for signal processing, instrument control and coordination. Connections between the microwave equipment and the Gigabox antennas are made using standard coaxial cables.

In many experiments, it is necessary to be able to "read" and "write" signals from the same port. Manually switching coaxial cables from the PSG to the DSO is 
slow and can destroy reconstructions, so we used four HP $8762 \mathrm{C}$ coaxial switches to reroute signals as required. The relative connections of the hardware and a diagram of the available connections on the box are shown in Figures 3-1 and 3-2.

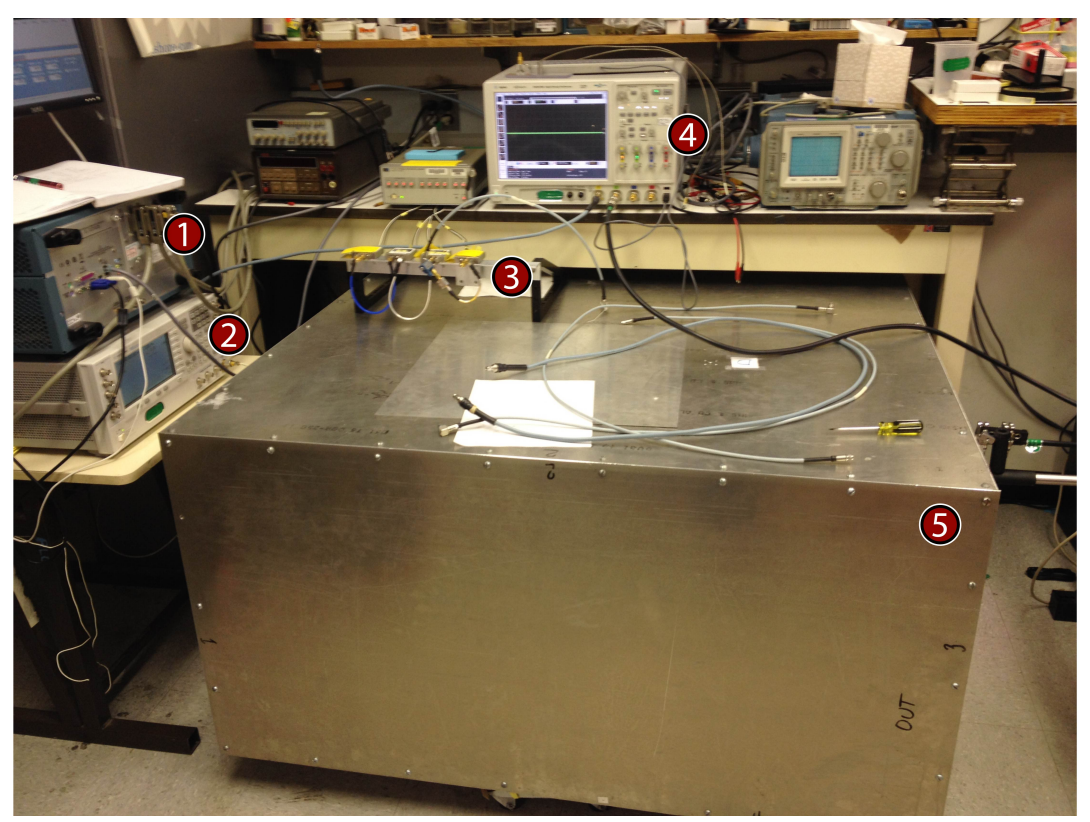

Figure 3-1: Experimental setup used: (1) Tektronix AWG7052 Arbitrary Waveform Generator, (2) Agilent E8267D Vector PSG Microwave Source, (3) Array of four Hewlett-Packard 8762C coaxial switches, (4) Agilent DS091304A Digital Storage Oscilloscope, 5) $1.06 \mathrm{~m}^{3}$ aluminum "Gigabox" with interior conductive scattering paddle.

TR processes fall into two main categories, linear and nonlinear. Linear TR (LTR) refers to any experiment that uses a single frequency for the interrogation pulse, time reversed sona, and reconstruction. Nonlinear TR (NLTR) makes use of harmonic reflections from the target to provide a means for isolation and targeting, a process similar to how we would envision a TR based WPT system to work. It is experimentally much simpler to create reconstructions with LTR than with NLTR. For this reason, these experiments are conducted in the absence of nonlinear elements, which respond to a fundamental frequency by generating harmonic signals. 


\section{DIMENSIONAL}

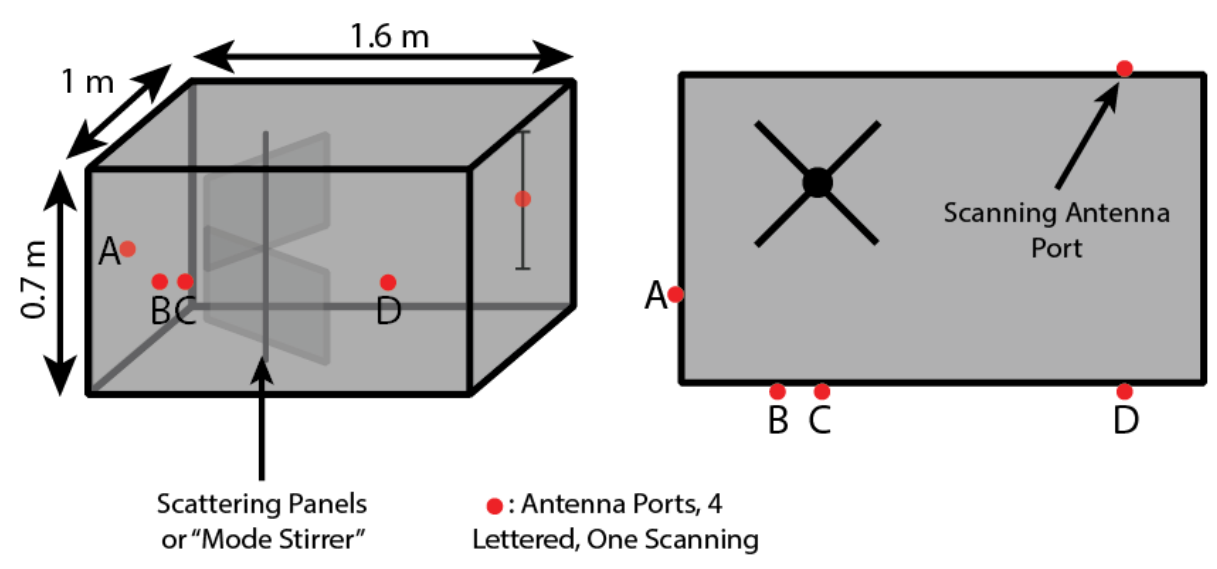

Figure 3-2: Diagram describing the internals of the Gigabox and the layout of its five antenna ports.

Conceptually, our general process for LTR in the Gigabox is as follows: we broadcast a Gaussian interrogation pulse from one port, serving as a transmitter. This interrogation pulse reverberates and echoes around the reflective cavity. Another port, designated as the receiver, records the multipath sum of these reverberations with the oscilloscope. This summed signal is the sona, and inherently contains information relating to the size and shape of the interrogation pulse, the location of any scattering media in the environment, and the location of both ports. That sona is reversed in the time domain and subsequently rebroadcast from the transmitting port. The signal will travel through the same multipath channels and reconstruct a time reversed version of the original interrogation pulse upon the receiver, with some additional noise. This process is illustrated in Figure 3-3. 

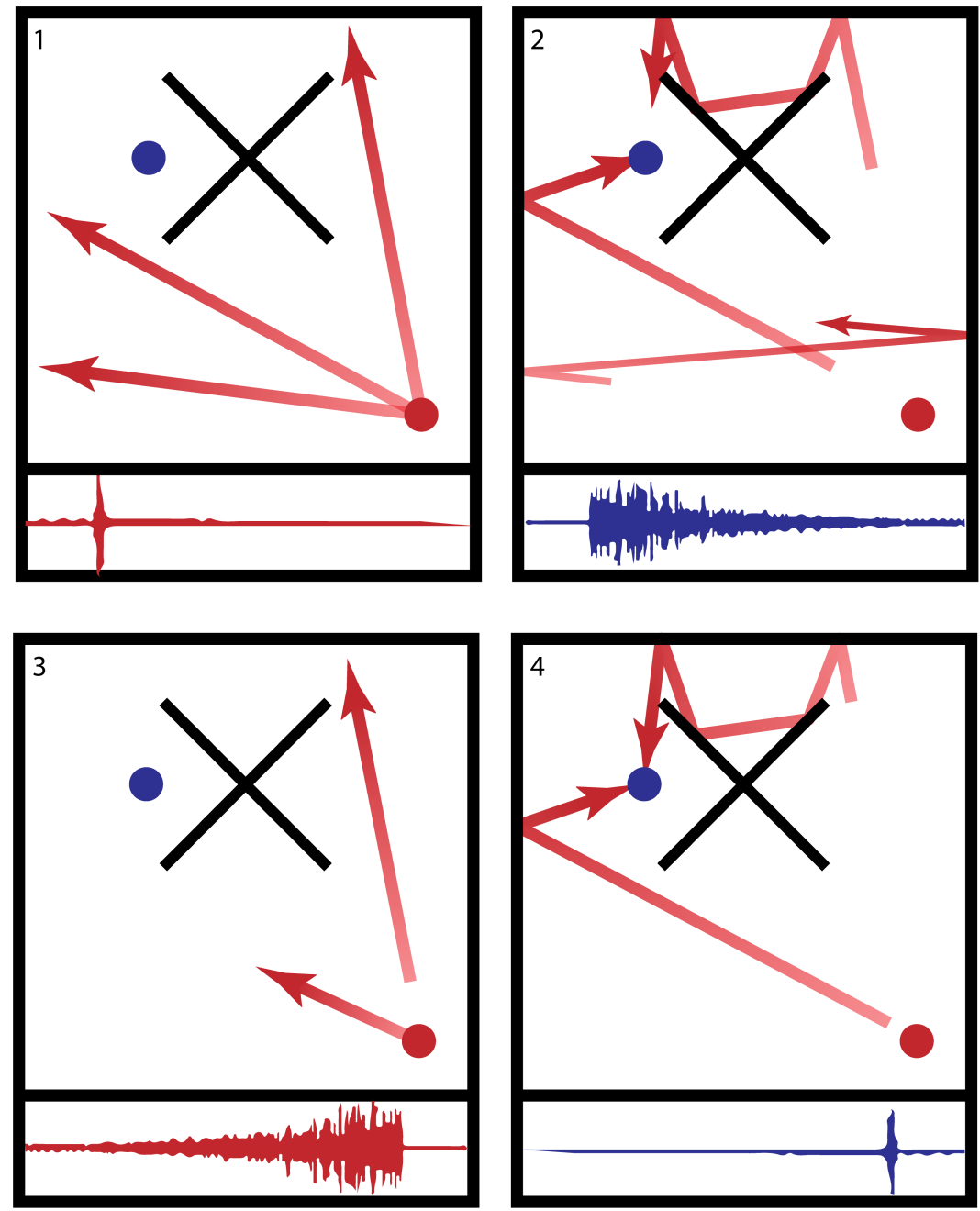

Figure 3-3: Reading in order from top left: Visualization of the linear time reversal process (1) The TRM broadcasts a signal (in this case, a pulse, pictured in the inset below the panel) into the cavity, which (2) reverberates within the cavity. Some of the reflections incoherently reach the receiver as the sona, represented in blue in the inset. (3) The TRM time reverses the sona, then re-emits it into the cavity. (4) The time reversed waves coherently collapse back on the receiver in a slightly distorted reconstruction of the original pulse (inset, blue). 
The experiments, results, and discussions for these sections are presented in the following sections. The modifications of the basic LTR methodology are discussed, as are the implications for the use of TR in a WPT system. The experience the team gained by performing these experiments and formulating results was extremely valuable in constructing and performing our later work with Nonlinear Time Reversal (NLTR). They also demonstrate important conclusions regarding the transmission of large amounts of energy to the receiver. 


\subsection{Overlapping Reconstructions}

\subsubsection{Purpose}

Frazier et al. demonstrate that the sona of a given interrogation pulse is significantly longer than the pulse that generated it [4]. This stands to reason, given that the sona represents reflections of the initial pulse shifted in time by differing path lengths.

To start with, an important feature of this method is spatial fidelity: the ability to deliver highly localized reconstruction to a reciever. In the interest of delivering more energy with each packet, there is a desire to make the duration of the reconstruction longer. To do this, however, would reduce the bandwidth of the interrogation pulse and reconstruction. The bandwidth of the interrogation pulse translates to the number of eigenmodes available for comunication between the transmitter and reciever. The requirement for a suitibly large bandwidth restricts the duration or temporal localization of the interrogation pulse and reconstruction. As a result, increasing the energy delivered per reconstruction packet by extending the duration of the interrogation pulse is not a favorable option as it reduces the spatial fidelity of the reconstruction.

To increase the amount of energy transmitted, we propose two modifications to the time reversal scheme. Either the amount of energy contained within each packet can be inflated, or the frequency of packets can be increased. Increasing the size of the reconstruction is a trivial matter; by amplifying the time reversed sona be- 
fore reinjection, the reconstruction is correspondingly amplified. We desire to send reconstructions more frequently than the length of the sona will allow. However, transmitting multiple sonas at once from the same antenna is possible-copying and shifting the signal in time can allow several signals to be sent in less time than the sum of the individual sona time lengths. This will in turn result in multiple transmitted reconstructions, and improved power transmission.

A concern with the above method is whether or not it would result in lost information and consequently degraded reconstruction quality. Sonas are sinusoidal signals-if overlaid, they will interfere constructively and destructively. Destructive interference may result in the "deletion" of transmission paths, reducing the efficiency of the TR process and decreasing the fidelity of the created reconstructions. In this experiment, we are interested in understanding the degree to which superimposing sonas will degrade the ability to transmit energy between ports using linear time reversal.

\subsubsection{Methodology}

This test sought to evaluate the practicality of overlapping sonas as a method of transmitting power. This experiment was done using the basic, two-port linear time reversal scheme, first described in Section 3.2. A diagram of this scheme appears as Figure 3-4.

A sona was generated with a $3.9 \mathrm{GHz}$ interrogation pulse. To verify that the sona could converge on the target, it was time reversed without any further manipulation, and injected back into the cavity. The reconstruction was recorded and compared to the original interrogation pulse to check for irregularities. 


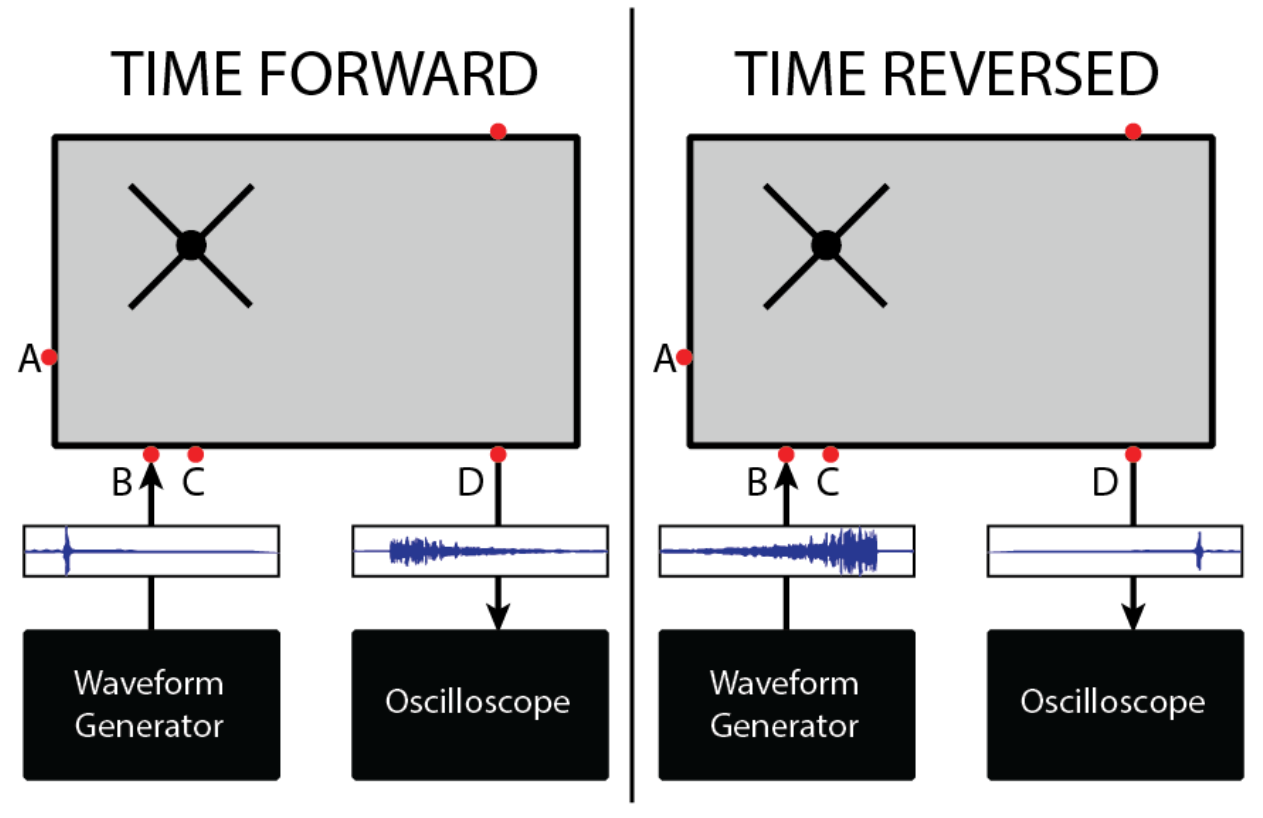

Figure 3-4: A diagram of our typical two-port linear time reversal scheme. In the time forward step, a pulse is broadcast from antenna port B and a sona collected at D. In the time reversed step, the reversed sona is broadcast from B and a reconstruction occurs at D. This setup takes advantage of spatial as well as temporal reciprocity to avoid having to record and broadcast from the same port, which greatly complicates cabling and introduces losses from the switches.

Once the efficacy of the single sona was established, the experiment was modified.

Several sonas were superimposed with a constant time shift, resulting in evenly spaced copies of the verified sona across the total broadcast window. Figure 3-5 gives an example of sonas overlapping in this way. The resulting signal was then time reversed and broadcast through the linear system in the same manner as before, which resulted in the reconstructions in Figure 3-6. This test was repeated many times, with the number of sonas superimposed within the $15 \mu$ s broadcast window varying from one to three hundred, which corresponds to repetition rates ranging from $66 \mathrm{kHz}$ to $20 \mathrm{MHz}$, respectively. The sonas and corresponding reconstructions of two samples from this range, 50 and 300 overlapped sonas, are shown in Figures 3-7 and 3-8, respectively. 


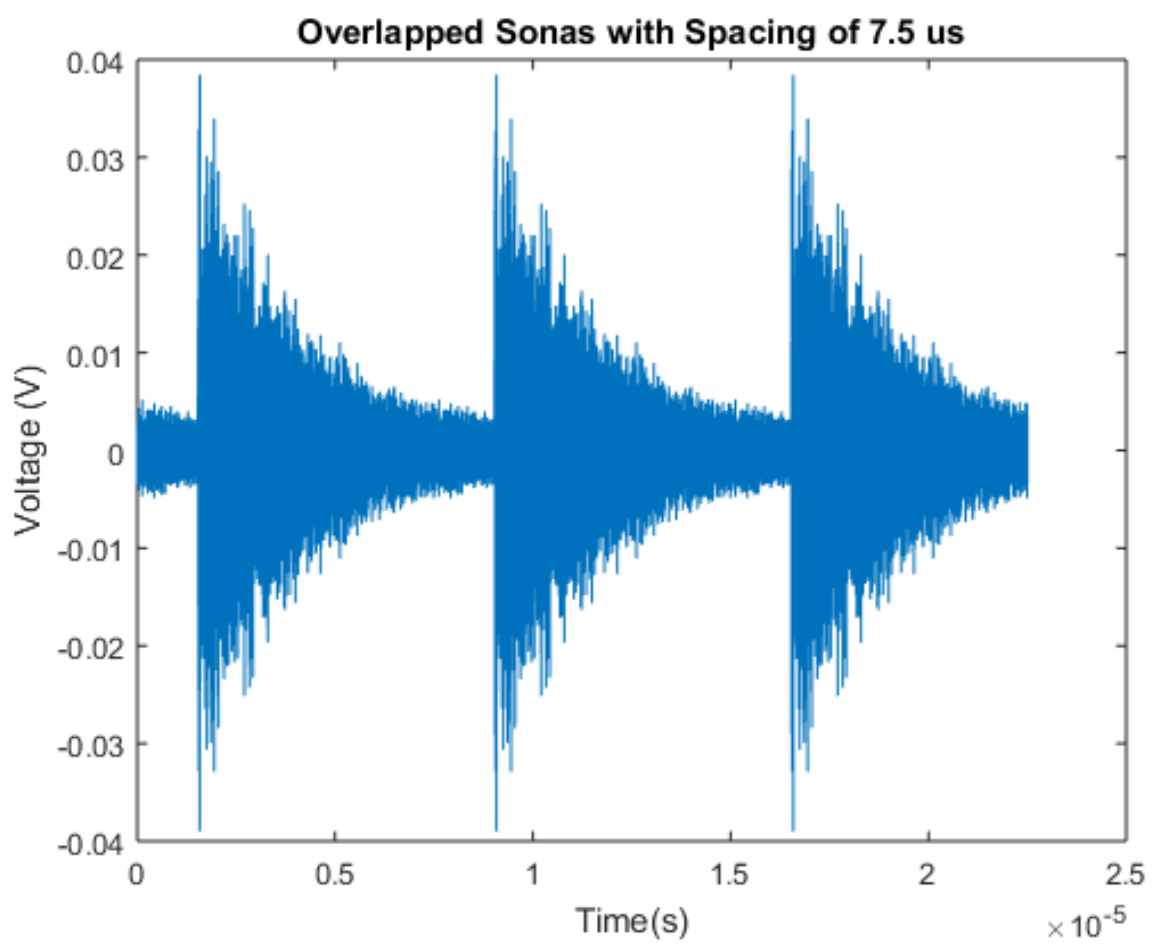

Figure 3-5: Overlapped sonas with a spacing of $7.5 \mu \mathrm{s}$.

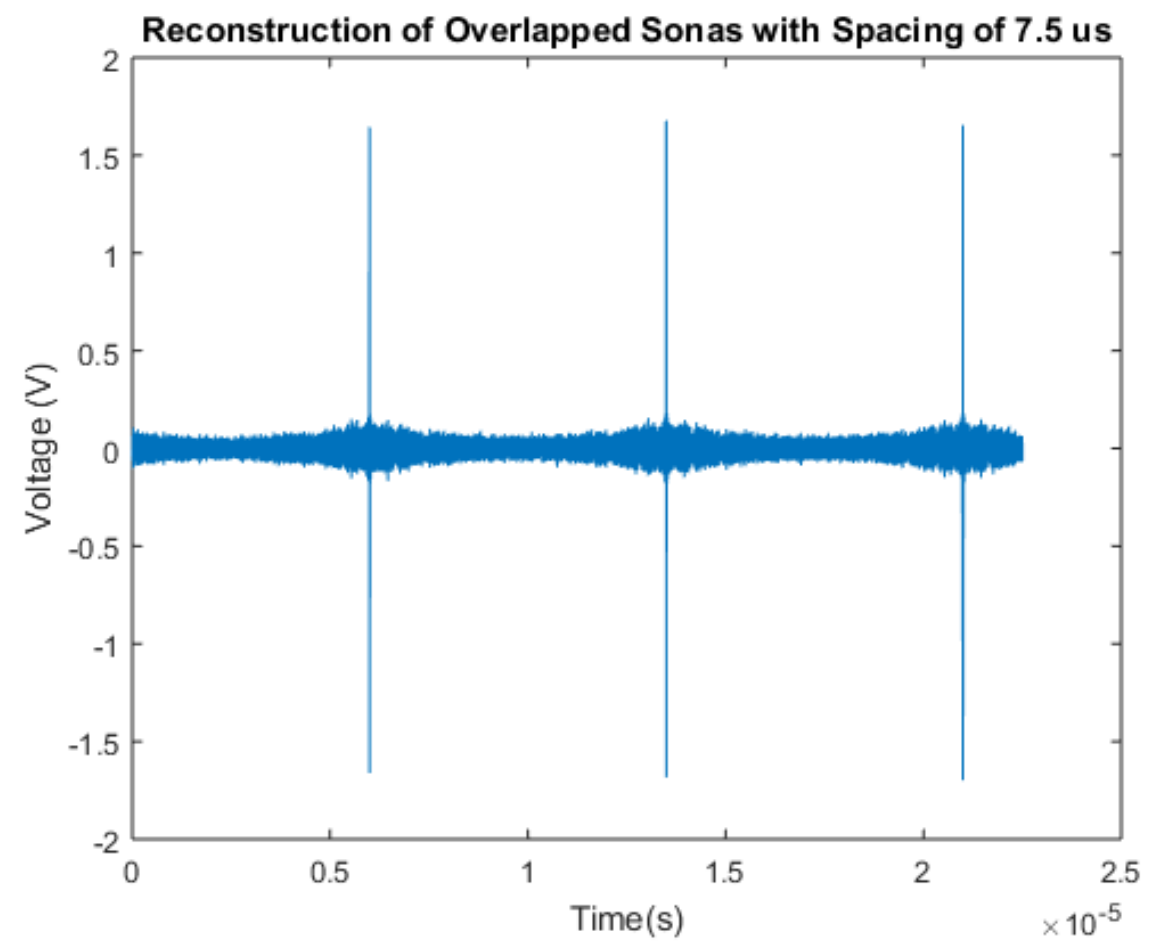

Figure 3-6: Reconstructions resulting from the sonas in 3-5. 


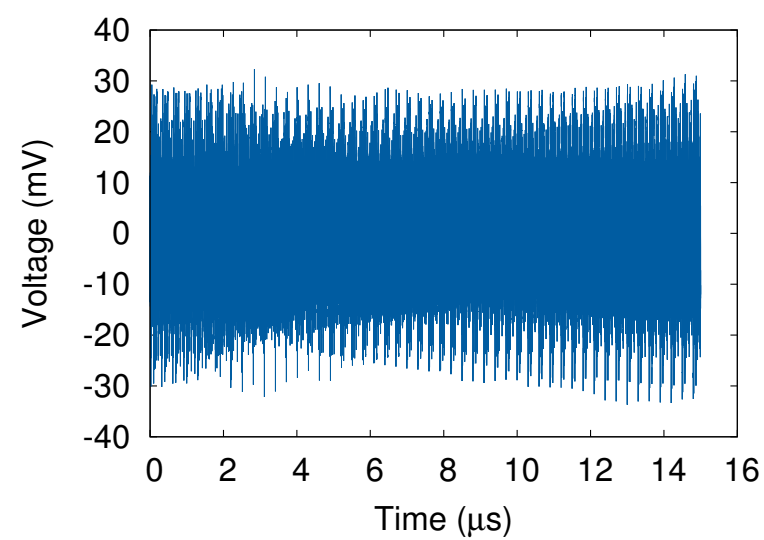

(a) Sonas

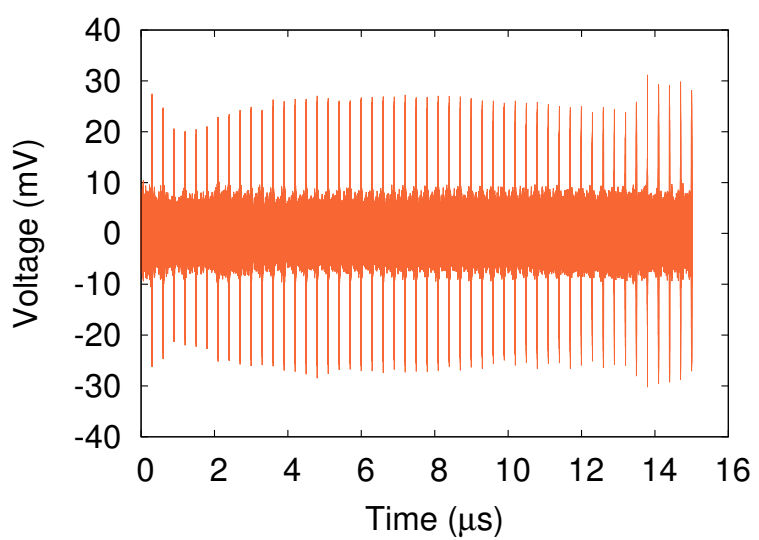

(b) Reconstructions

Figure 3-7: 50 sonas superimposed within the $15 \mu$ s broadcast window, corresponding to a repitition rate of $3.33 \mathrm{MHz}$.

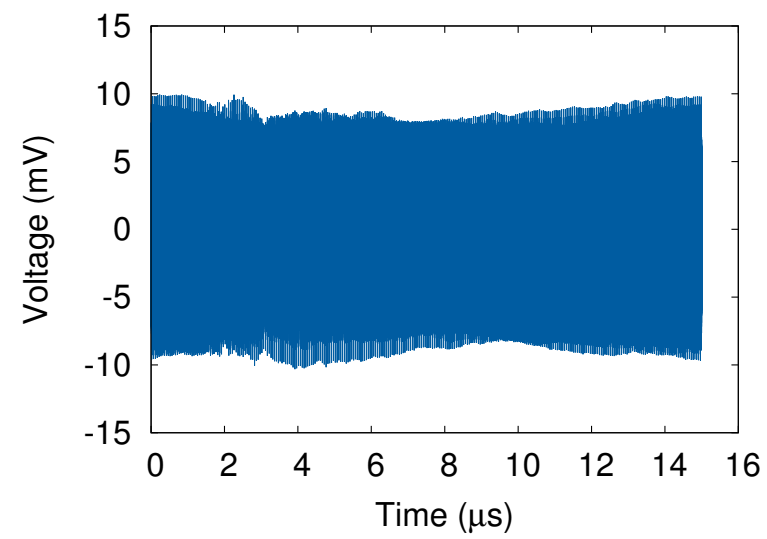

(a) Sonas

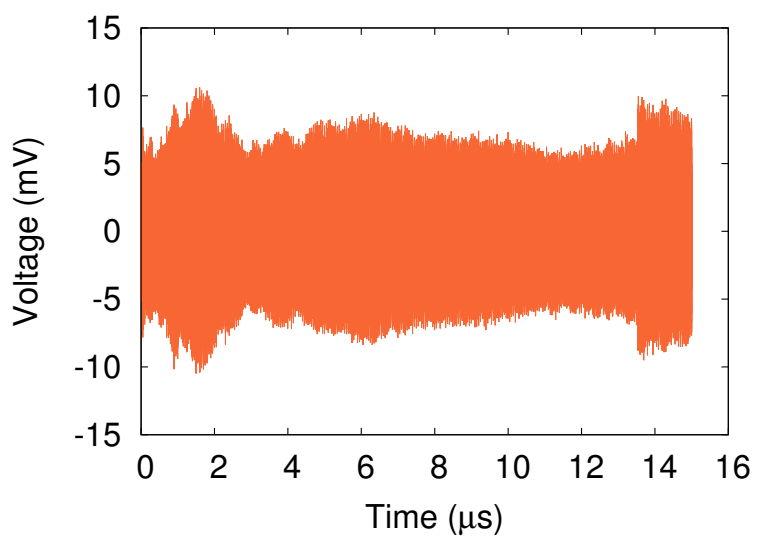

(b) Reconstructions

Figure 3-8: 300 sonas superimposed within the $15 \mu$ s broadcast window, corresponding to a repitition rate of $20 \mathrm{MHz}$. 


\subsubsection{Results}

Increasing the number of sonas should increase the amount of power transmitted in a given time frame, if the amplitude of each reconstruction remains constant. We hypothesized that overlapping reconstructions by linearly superimposing sonas would not decrease the size of the reconstructions. As a result, a great amount of effective power was transmitted per duty cycle. Our experiments seemed to support this conjecture, in that the number of reconstructions in the cycle could be increased while maintaining their characteristic shape. However, the size of the packets did decrease as the number of reconstructions within the window became larger.

We believe these results to be a combination of two factors. The loss of information due to destructive interference during sona superposition, and the function of power scaling within the PSG signal generator both may have contributed to this decreasing trend. The generator attempts to level a preset amount of power across the entire broadcast window, which is split among the multiple reconstructions. To better model how these reconstructions would interact with a practical WPT system, we also examined the "total peak-to-peak voltage," which we defined as the maximum peak-to-peak voltage multiplied by the number of reconstructions present within the measurement window. This quantity emulates the energy that would be rectified within the same time window, so we can compare the various repetition rates with regard to the energy that a receiver would accept within that time frame. This correlation is plotted in Figure 3-10.

Important to note is the overall increasing trend, which resembles a square root 
function. This is expected, as the power is expected to vary linearly with the number of overlaid sonas, and is proportional to the peak-to-peak voltage squared. The positive correlation between repetition rate and total voltage indicates that the use of this technique would be very valuable for a WPT scheme. We can acquire a sona and then broadcast the time reversed version at very high repetition rates to transmit large amounts of energy to the receiver. Coupled with an arbitrary amplification according to the application's needs, this modification to a time reversal WPT system shows promise for increasing the quantity of power that can be transferred. 


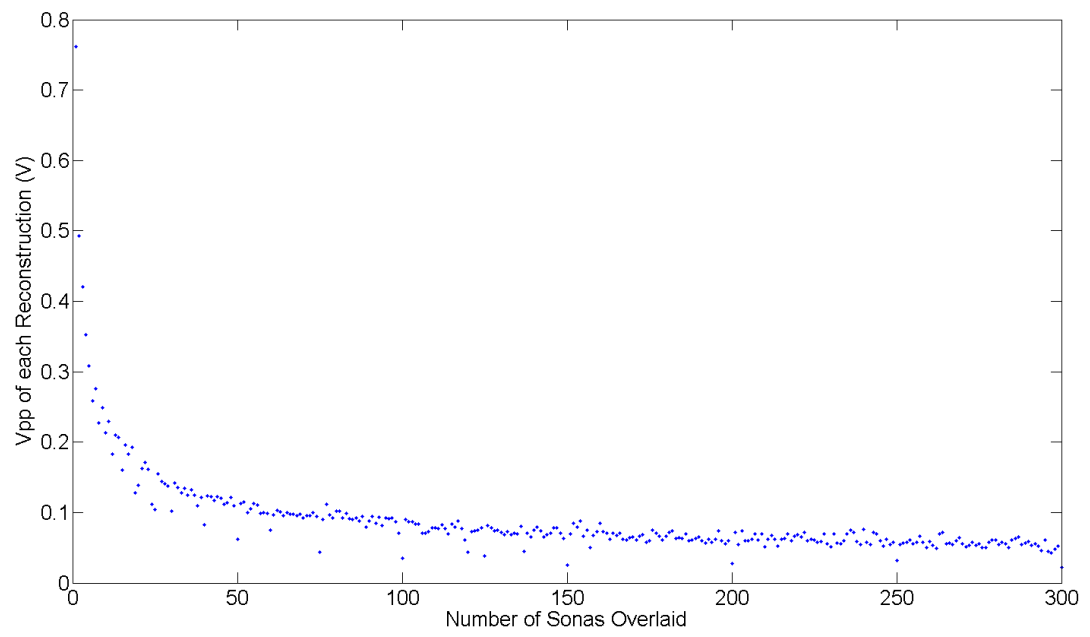

Figure 3-9: Max peak-to-peak voltage versus number of overlapped reconstructions.

Voltage At Receiver as a Function of Overlapped Sonas

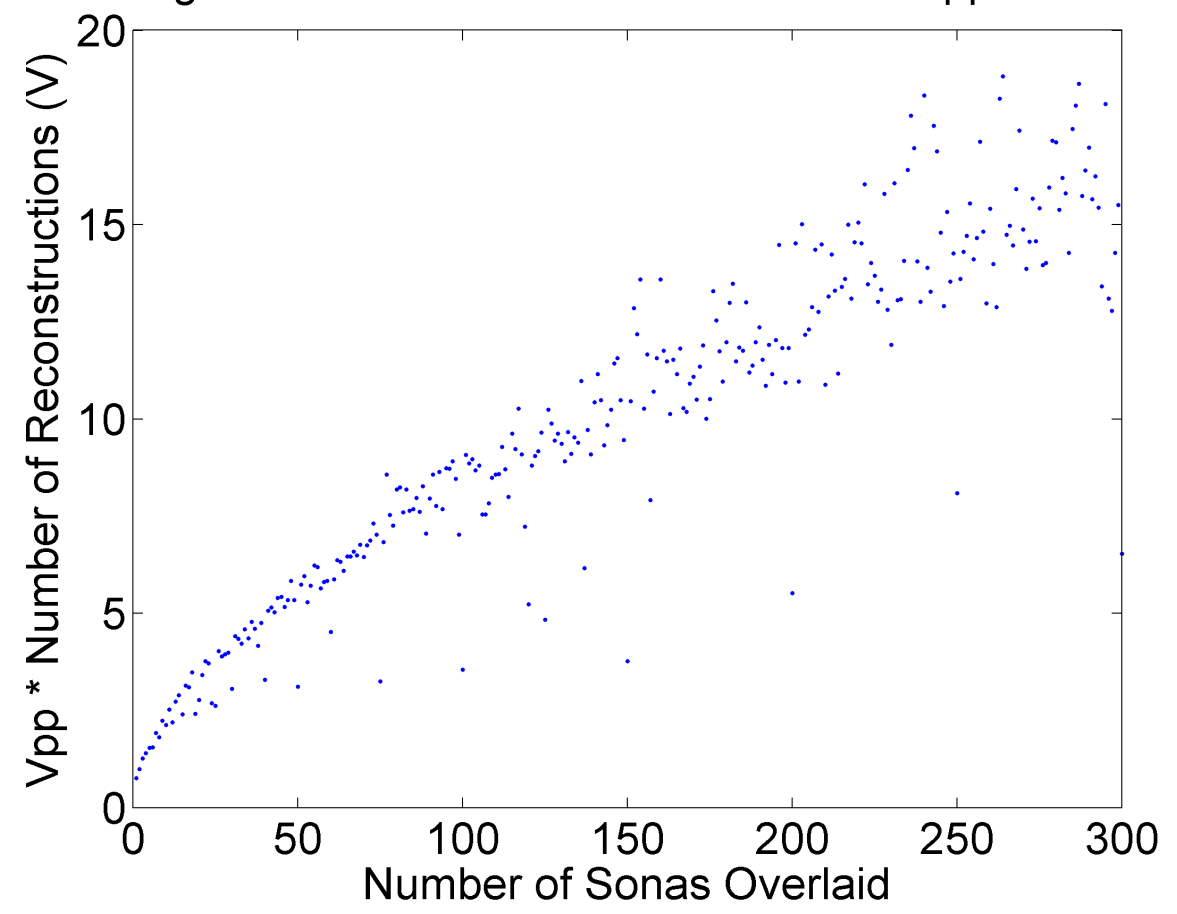

Figure 3-10: Total peak-to-peak voltage versus number of overlapped reconstructions. 


\subsection{Moving Reconstructions}

\subsubsection{Purpose}

The TR process assumes that the environment remains fixed between the timeforward and time-reversed steps. It also assumes that the source and target remain fixed between these two steps. However, in a practical WPT system it is desirable to support a receiver in motion. This ability gives a convenient benefit to the consumer, who would not be limited to keeping their mobile device stationary for charging. This is a gap in the current market, which TR may be able to fill. In this section, we propose and investigate a method for using the broad spatial voltage profile to provide a consistent voltage to a receiver in motion.

The experiment is conducted in two parts: mapping the spatial voltage profile of a TR reconstructed signal, and iteratively updating the target location of the time reversal mirror. The first part of the experiment intends to consider the extent to which a reconstruction is localized in space. This property has implications for several parts of the WPT scheme including system efficiency, health concerns, and tolerance in receiver position relative to the target location. A broad reconstruction in space would relate to a lower efficiency, because only a fraction of the energy that is being sent is collected by the receiver. Consequently, if the reconstruction is broad then there is a possibility that some energy could be sent to an unintended nearby medium; for example, the target device might be in a user's pocket, and a broad profile could indicate that the user themself would be absorbing some of that energy. While both 
of these effects are generally things that we would like to avoid in a WPT system, it also could facilitate the accommodation of a moving receiver. A profile that is not particularly localized would allow the receiver to shift slightly from the target location and still collect a significant portion of the energy packet that was sent. When the voltage level at the receiver's position drops below some acceptable level, the TR process could be performed again. This would update the target location to the receiver's current location, and reset the voltage at the receiver to the peak value of the reconstruction. A broader reconstruction in space would allow this process to be performed less frequently, while a narrower reconstruction may greatly limit the speed that the receiver could travel at.

\subsubsection{Spatial Profiling}

\section{Methodology}

The setting for this experiment differs only slightly from the one described in Section 3.2: now, the receiving antenna is mounted on a PI MikroMove M-415.DG translation stage, shown in Figure 3-11, which allows the cavity to remain sealed during translation. Initially, the receiver is moved to the middle of the scanning range (denoted position 0 ). An interrogation pulse is used to capture a sona and begin creating reconstructions at this initial position. The receiver is then moved from the bottom $(-35 \mathrm{~mm})$ to the top $(+35 \mathrm{~mm})$ of the scanning range, stopping every $0.2 \mathrm{~mm}$ to measure and record the peak-to-peak voltage of the reconstruction (still focused on 0) at each point. 


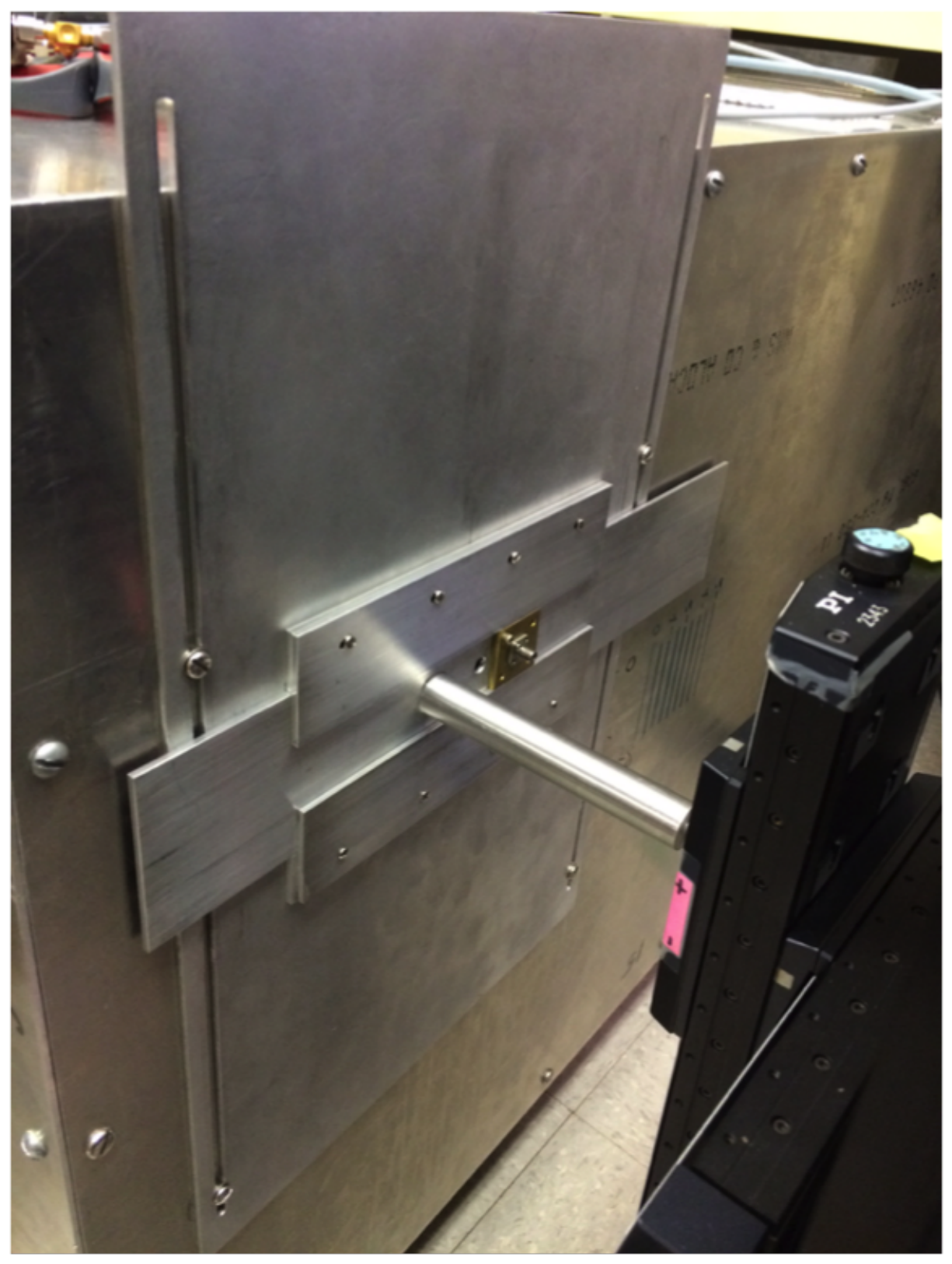

Figure 3-11: The PI MikroMove translation stage (black, right) attached to a sliding window on the Gigabox (left) via a metal arm. The antenna is attached to the sliding window just to the right of the arm. The MikroMove translates the arm up $(+)$ and down (-), which moves the sliding window and ultimately the antenna along the same range of motion. This particular model had a maximum range of motion of $70 \mathrm{~mm}$ along a single axis. 
Results

We repeated the experiment described above for carrier frequencies in the range 4-9 GHz and display these results in 3-12. Differences in amplitude between the curves are due to differences in $S_{1,2}$ parameters between frequencies for our particular antennas.

Based on observations from the results in Figure 3-12, we expect the reconstruction peak-to-peak voltage profile to take the form of a $\operatorname{sinc}(x)$ function about the target location [31]. Thus, we propose the following equation to predict $V(x)$, the maximum peak-to-peak voltage from a given reconstruction, as a function of $x$, the distance between the reconstruction focal point and the receiver:

$$
V(x)=a \cdot\left|\operatorname{sinc}\left(\frac{x+c}{b}\right)\right|+d
$$

where $a$ is the maximum peak-to-peak reconstruction amplitude, $b$ is the halfwavelength, $c$ is the location of the antenna along the x-axis, and $d$ is the noise level offset voltage. Since $b$ is proportional to the wavelength (and inversely proportional to frequency), as the carrier frequency is increased, $\frac{1}{b}$ also increases, causing the "bubble" of the sinc function in Figure 3-12 to get smaller. This relationship is shown explicitly in the inset of Figure 3-12. Figure 3-13 shows Equation 3.1 fit to the $5 \mathrm{GHz}$ curve from Figure 3-12, including error bars. The fit qualitatively looks good around the primary peak, but quantitatively has a high reduced $\chi^{2}$ value. We believe this is primarily due to the large background noise level that has hidden the secondary peaks. Fitting to just the primary peak produces a much better goodness of fit metric. The error bars are primarily systematic, introduced by the oscilloscope 


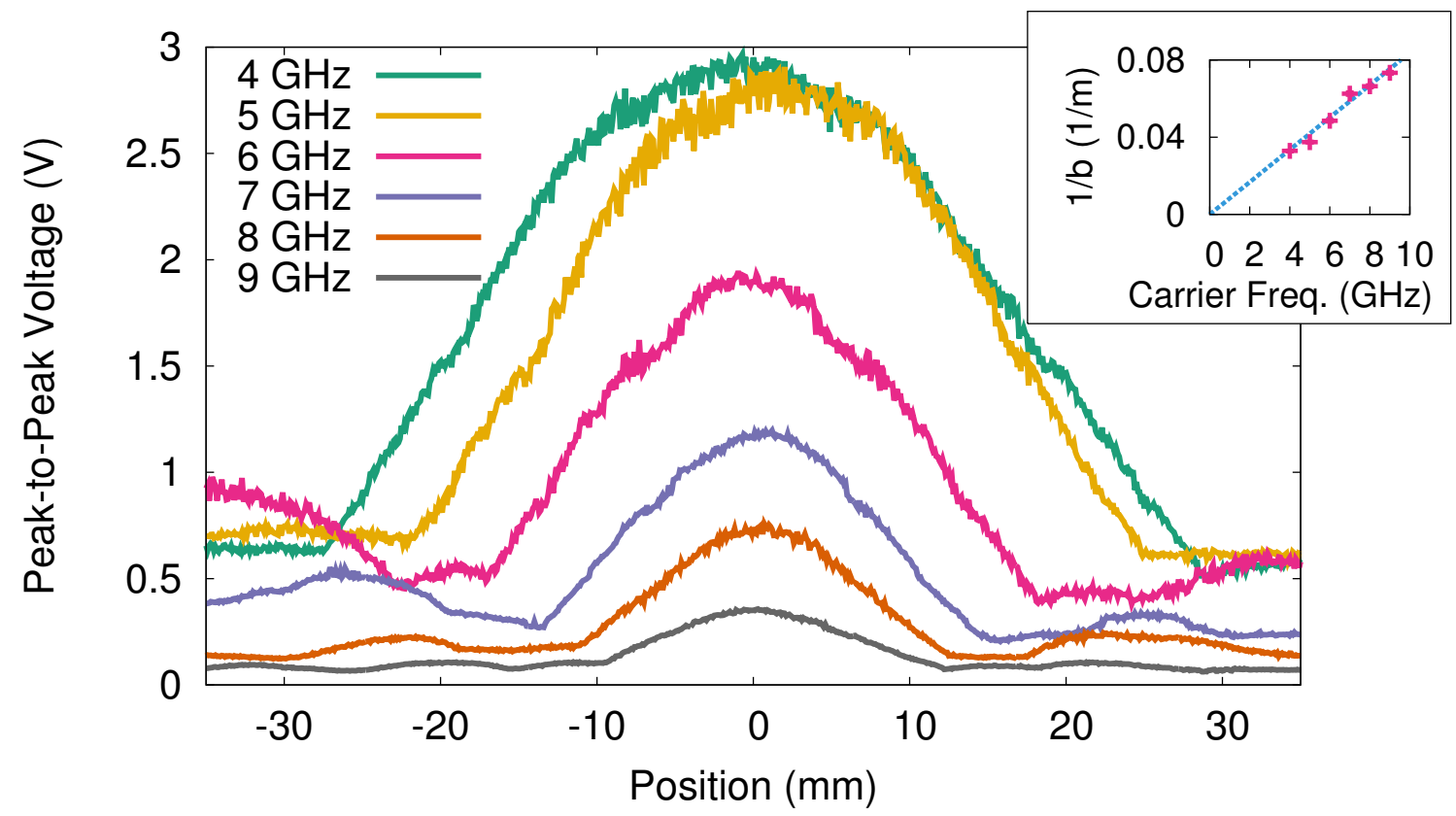

Figure 3-12: Spatial profile of peak-to-peak voltage of reconstructions investigated at carrier frequencies ranging from 4 to $9 \mathrm{GHz}$ in $1 \mathrm{GHz}$ steps. The inset shows the inverse of the fit $b$ values versus carrier frequency, showing the expected linear relationship.

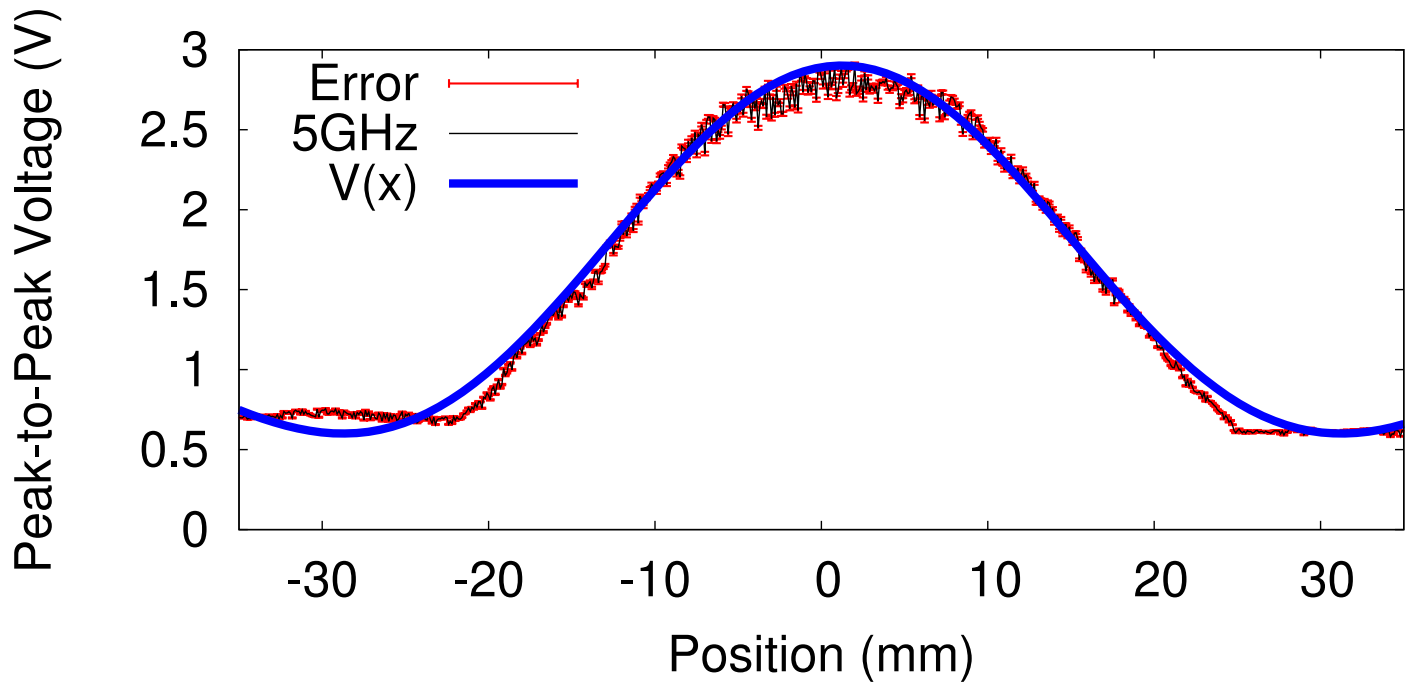

Figure 3-13: Measured peak-to-peak voltage of reconstructions received in the vicinity of a time-reversed wave collapse location with a $5 \mathrm{GHz}$ carrier frequency, and fit to the sinc(x) function. The parameters here are $a=2.18 \pm 0.046397869$, $b=26.72 \pm 0.134629783, c=-73.58 \pm 0.116338652$, and $d=0.43 \pm 0.012711137$. The fit parameters for the remaining 4-9 GHz frequency profiles from Figure 3-12 are given in Appendix A. 
internal voltage multiplier used in scaling.

The results presented in Figure 3-12 allow us to draw several conclusions. In this frequency range, the reconstructions can be categorized as broad, spanning several centimeters in width. This is significant because it indicates that we can move the receiver slightly away from the target location and still collect energy from the reconstruction. This allows us to implement a system targeting a moving receiver.

\subsubsection{Reconstructing on a Moving Target}

\section{Methodology}

For this experiment, the receiving antenna was moved at a constant speed of $0.2 \frac{\mathrm{mm}}{\mathrm{s}}$ across the entire $70 \mathrm{~mm}$ range provided by the translation stage. To counteract the degradation of reconstruction strength as the antenna moved, we periodically repeated the interrogation step, effectively re-centering the reconstruction on the antenna. Since the test equipment does not allow broadcast of one sona while collecting another, it was not possible to transmit power during the collection time, leading to a finite "dead time," denoted $t_{d}$ in Figure 3-14. During the broadcast period, the time-reversed sona was continually broadcast into the cavity (once every $15 \mu \mathrm{s}$ ) and the peak-to peak voltage across the receiver was measured once every 2.05 seconds, meaning that the reconstructions are highly undersampled in this plot. After every 15 samples were collected, the process was repeated. We refer to this full process

of collecting a new sona and then broadcasting it for a given period time as a full "cycle" of length $t_{c}$. The results in Figure 3-14 below were obtained using a carrier 
frequency of $5 \mathrm{GHz}, t_{d}$ of 7 seconds, and $t_{c}$ of 39.8 seconds. Based on the results from Section 3.4.2, the peak-to-peak reconstruction voltage measured by the receiver is expected to decay according to the $\operatorname{sinc}(x)$ function as the receiver moves away from the reconstruction focal point. This $\operatorname{sinc}(x)$ function will be centered on the position where the sona was last collected, making the reconstruction focus continually lag behind the antenna. Consequently, the maximum reconstruction strength is limited by the time needed to collect, time reverse and re-broadcast an updated sona. The following equation is proposed as a model for the peak-to-peak voltage of the reconstruction on a moving target as a function of time, assuming a constant velocity $\bar{v}:$

$$
V(t)=\left\{\begin{array}{lll}
0 & : t & \left(\bmod t_{c}\right) \leq t_{d} \\
a \cdot\left|\operatorname{sinc}\left(\frac{\bar{v} t}{b}\right)\right|+d & : t & \left(\bmod t_{c}\right)>t_{d}
\end{array} .\right.
$$

Results

The results of this experiment are very promising. We hypothesized that the reconstruction peak-to-peak voltage would reset back to a maximal value when a new sona was used to refocus the reconstruction, and follow the voltage profile as the receiver moved away from the new target location. This was almost exactly confirmed by the experimental results. This is encouraging for the application of TR to a practical WPT scheme because it proves the applicability to a moving receiver system.

It is worth noting that the speed of the receiver is relatively slow at $0.2 \frac{\mathrm{mm}}{\mathrm{s}}$. This 


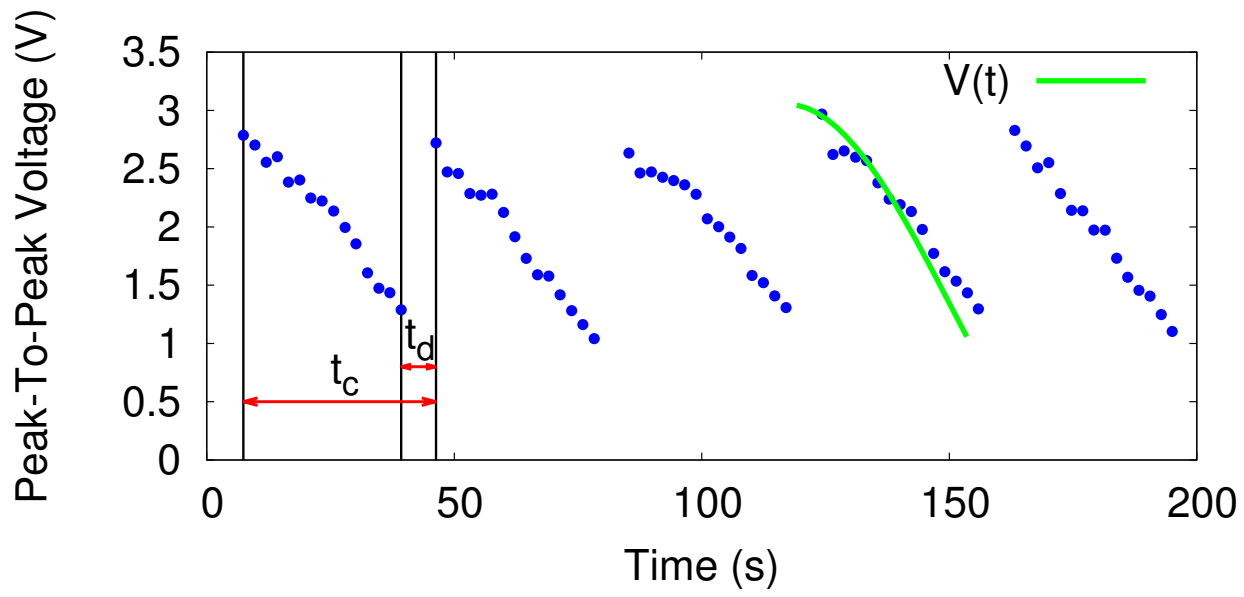

Figure 3-14: Reconstruction peak-to-peak voltage vs. time as the target moves along one wall of the enclosure. A new sona signal is acquired every $t_{c}=39.8 \mathrm{~s}$, leading to a dead time of duration $t_{d}=7 \mathrm{~s}$. The target is moving at a speed of $0.5 \frac{\mathrm{mm}}{\mathrm{s}}$ and the carrier frequency is $5 \mathrm{GHz}$. The green line is Eq. 3.2.

experiment is intended to be a proof of concept, with generalized equipment that carries significant overhead. The GPIB connections between the signal generators, oscilloscope, and workstation contributed to the long delay between system operations, in addition to the slow processing speed of the equipment itself. We expect that with a dedicated time reversal system, the TR process of reacquiring a new target location could be performed in milliseconds and describe the ramifications for doing so in Section 3.5. This would greatly increase the maximum receiver speed that could be accommodated as the reconstruction could be refocused more often, and thus the average peak-to-peak voltage could be raised closer to that maximal value. We conclude that, with further research and engineering work, a TR WPT scheme could feasibly power a moving receiver in realistic scenarios. 


\subsection{Timing and Computational Complexity}

In Section 3.4, we showed that the ability of a time reversal system to transmit energy to a moving target is dependent on the spatial profile and transmission dead time, $t_{d}$. In this work, our equipment limited us to a $t_{d}$ of 7 seconds, which would be too slow to handle most typical movement, such as walking across a room. The primary bottlenecks here were the communication between the oscilloscope and computer, and the matrix operations performed in MATLAB on the computer. Figure 3-15 details the amount of time taken by each step of the time reversal process, as viewed from the perspective of the central computer, where each bar can be categorized as either a result of communication overhead or software operations.

A practical system could overcome these limitations by combining the two components into a single device, which would remove the communication overhead, and optimizing for the specific matrix computations in hardware. Once a sona has been recorded (shown in Figure 2-8b to be on the order of $15 \mu \mathrm{s}$ ), the theoretical lower bound on processing time would be limited only by the amount of time required to compute the matrix operations.

The careful reader may notice that we report a dead time of 7 seconds in the previous section, but that the process appears to take roughly 13 seconds in Figure 315. This is a result of the fact that we made a few minor optimizations to the process after measuring the timing in this plot, but never re-generated a new plot. Specifically, these optimizations included: setting up the AWG and PSG once at the beginning of the process (as opposed to setting them up within each iteration of the TR process), 
and removing the 1 second sleep time for each of the switches, as we found that they actually moved fast enough to simply continue the code immediately. Together, these optimizations accounted for roughly 6 seconds of the process, bringing us down to the reported dead time of 7 seconds. However, it is worth repeating that these minor "optimizations" (or rather corrections) were done ontop of highly unoptimized general TR code, meaning there is much room for decreasing the time further. 


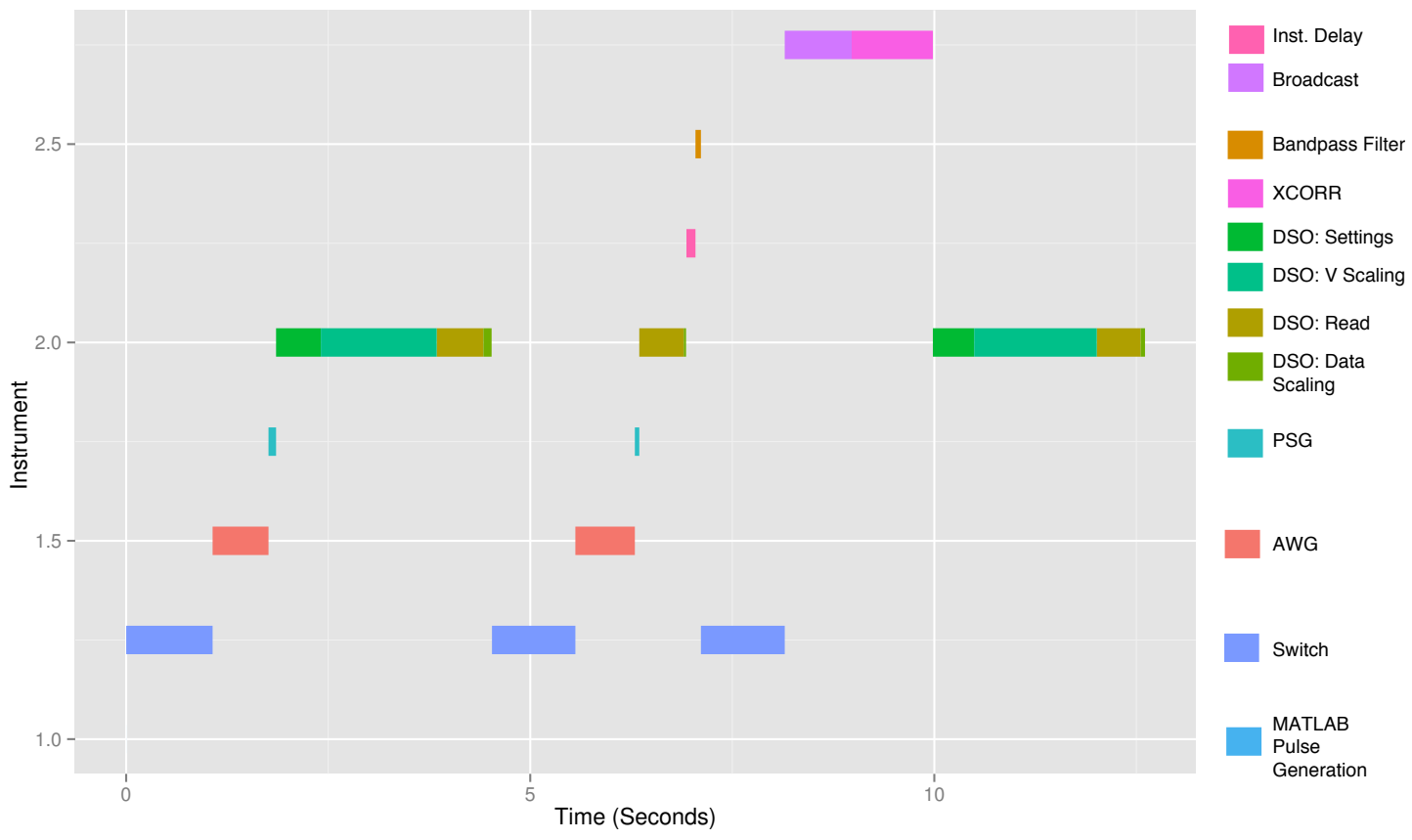

Figure 3-15: This plot shows wall clock time elapsed for rate limiting steps in the MATLAB code which controlled all of our operations and equipment in the time reversal process (measured using MATLAB's tic and toc functions). Bars for Switch, AWG, PSG, and DSO:Read are primarily a result of communication between the devices, while XCORR, Bandpass Filter, Broadcast, and the remaining DSO operations are simply software operations taking place in MATLAB. However, it is worth noting that the remaining DSO operations were incorporated to handle a wide variety of experiments and not optimized for this process. Code and hardware optimized specifically for this purpose could make certain assumptions about the recording process and should be able to eliminate these steps altogether. Finally, it is also worth noting that, again due to communication delays, it was sometimes necessary to hardcode a sleep delay in the MATLAB code, further increasing the total time of the process. 


\subsection{Transfer Range}

Our experiments throughout this chapter thus far have successfully demonstrated wireless power tranfer via time reversal in a relatively small encosure. A natural question one might ask at this point is: what if the enclosure were larger? Although experimentation inside of a larger enclosure was outside of the scope of this work, we can reason about how parameters of time reversal relate to the volume of the enclosure.

As waves propagate throughout the enclosure, they will be subject to some amount of loss each time they reflect off of a surface. Here we make the simplifying assumption that metallic losses are the domininant loss mechanism of our enclosure. Then, we can define the quality factor $Q$ of the enclosure to be:

$$
Q=\frac{V}{S \delta}
$$

where $V$ is the volume of the enclosure, $S$ is the surface area of the enclosure, and $\delta$ is the skin depth of the metal walls. The skin depth can be further defined to be dependent upon the resistivity of the metal, $p$ :

$$
\delta=\left[\frac{2 \rho}{\omega \mu_{0}}\right]^{\frac{1}{2}}
$$

Since surface area scales as $\frac{2}{3}$ the power of volume, we can rewrite 3.3 as:

$$
Q \sim \frac{V^{\frac{1}{3}}}{\delta}
$$

Now, we can describe how decay of energy in the cavity will scale as a function of Q: 


$$
U \sim e^{\frac{-\omega t}{Q}}
$$

We can simplify this into more familiar form by writing it has $e^{\frac{-t}{\tau_{\text {decay }}}}$, where decay time for energy scales as

$$
\tau_{\text {decay }} \sim \frac{Q}{\omega} \sim \frac{V^{\frac{1}{3}}}{\delta \omega} \sim \frac{V^{\frac{1}{3}}}{\omega^{\frac{1}{2}}}
$$

based on Equation 3.5, showing that as we either increase volume or decrease frequency, energy decay time will increase slowly.

In the context of time reverseal, the Heisenberg time $\left(\tau_{\text {Heisenberg }}\right)$ can be defined as the saturation time for sona measurement. Namely, it is the length of time after which collecting a longer sona will no longer result in a greater quality reconstruction. Conversely, collecting a sona significantly shorter than $\tau_{\text {Heisenberg }}$ will result in a poor reconstruction. The ability to capture a long-duration sona above the noise floor depends on environmental loss. The greater the loss, the more difficult it is to capture a longer sona signal, which will decay as described in Equation 3.6.

For any three-dimensional enclosure, the Heisenberg time can be quantified as:

$$
\tau_{\text {Heisenberg }}=2 \frac{2 \omega^{2} V}{\pi c^{3}}=10 \mu s[f(G H z)]^{2} V\left(m^{3}\right)
$$

where $\omega$ is the angular frequency, $V$ is the volume of the enclosure, and $c$ is the speed of light in the enclsoure. Thus, we can see that the Heisenberg time grows quickly with both frequency and enclosure volume, meaning that a larger enclosure volume will make it more challenging to collect a sona that will be of sufficient length for a high quality reconstruction.

For the most efficient and best quality reconstruction, we want to be in the limit 
$\tau_{\text {Heisenberg }}>\tau_{\text {decay }}$ so that the cavity allows us to fully capture energy from the sona before it has decayed. Thus, we want to maximize decay time without surpassing the Heisenberg time. Given these expressions for $\tau_{\text {Heisenberg }}$ and $\tau_{\text {decay }}$, we can see that a smaller volume enclosure and lower frequency will increase the decay time, but decrease the Heisenberg time. In our study, we did not calculate these values or attempt to optimize the process based on them, but quantifying these values and taking them into consideration would likely provide a noticable benefit in reconstruction quality and thus in ability to transmit power via TR.

Another way of looking at larger environments is to consider those that have openings. Sun Hong et al. found that a reconstruction could still successfully be created in an open semi-reverberant system (e.g. a cavity with large holes or cuts introducing significant loss at specific areas), which is much more representative of realistic environments. At a certain point, a large enough enclosure can be compared to an open system in which some surfaces (primarily those walls that are nearest the transmitter) will provide many usable paths, while others (far walls or cuts in the wall) will not provide any usable paths at all. The success of Hong's experiments suggest that TR should be capable of focusing signals in larger environments, assuming that enough reflective surfaces exist nearby to provide a sufficient number of transmission modes for the sona and reconstruction [32]. In Section 6.3.1, we further discuss the use of nearby reflective materialis in more lossy scenarios and suggest next steps for evaluating the method.

Although energy decay is certainly a necessary downside of any long-range WPT system, one positive aspect is that we expect as the room size is increased, the average 
energy to hotspots (concentrations of energy outside of the reconstruction point) will decrease, but further work is necessary to quantify to what extent, and whether it depends upon the volume, the number of modes, or something else entirely. 


\section{Chapter 4: Nonlinear Time Reversal}

The preceding chapters have been concerned with linear time reversal, or LTR, and have focused primarily on investigating properties of reconstructions. Now, we move into our nonlinear TR (NLTR) investigations, which focus on proof of concept of targeting capabilities. NLTR makes use of harmonic reflections from the target to isolate it via frequency domain inspection after the sona is collected, a process similar to how we would envision a TR based WPT system to work.

Conceptually, our general process is this: we broadcast a Gaussian pulse from one port. Elsewhere in the cavity is a nonlinear element serving as a target. Any signal that interrogates this element will generate higher frequency harmonics of the interrogation signal. Due to this aspect of the nonlinear object, our initial interrogation pulse will passively generate a harmonic signal. These harmonics are embedded along with all the other echoes in the sona collected back at the transceiving port. However, before time reversing the sona, we transform it into the frequency domain and filter out all the components not within some band of the harmonic frequencies. In this way, we isolate only those wave paths that had contact with the nonlinear target. That filtered sona is time reversed and rebroadcast from the transceiving port, which will cause a slightly distorted version of the original Gaussian pulse to reconstruct at the target. This process is illustrated in Figure 4-1. 

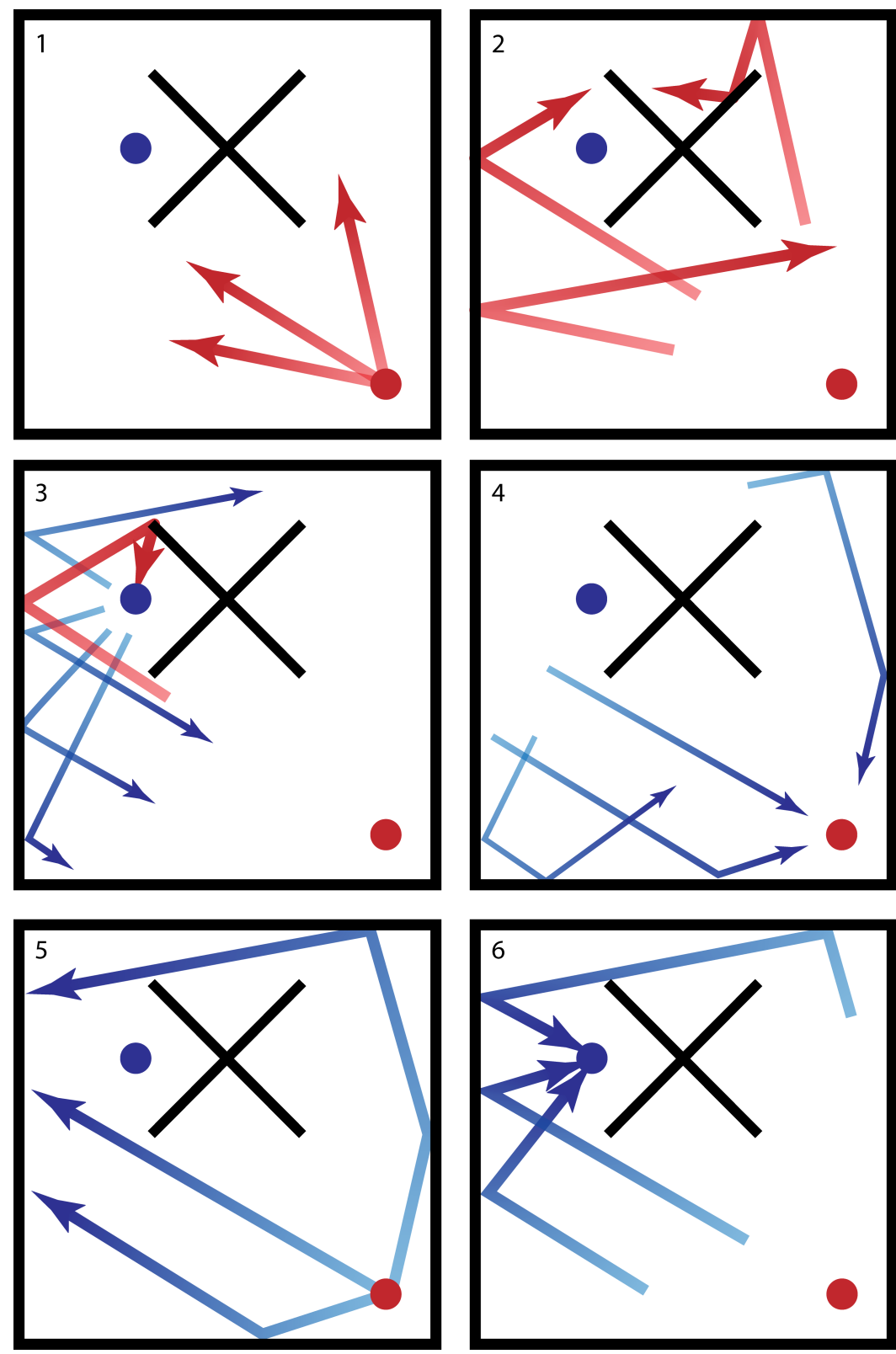

Figure 4-1: Reading order from top left: (1) The TRM broadcasts a signal into the cavity at one frequency, which (2) reverberates within the cavity. Eventually, (3) the signal reaches the nonlinear element somewhere within the chamber. Reflected waves that encounter this element have a characteristic frequency signature containing harmonics of the original signal. (4) Some of these harmonic reflections find their way back to the TRM. The TRM filters the sona to extract only those reflections, then time reverses and (5) re-emits them. (6) The time reversed waves collapse back on the nonlinear target. 
We explored several different applications of NLTR with an eye towards adapting the technique for WPT, both experimentally and in simulation. The experimental portions met with limited success due to the difficulty of passively producing harmonics of significant magnitude. We attempted to use both a diode as an electric nonlinear object due to its nonlinear I-V curve as well as ferromagnetic nanorods as a magnetically nonlinear object due to the nonlinear magnetization curve. In both instances, the nonlinear response we obtained was quite small. Due to this limitation, our NLTR experiments took place entirely within the realm of numerical simulations. 


\subsection{Experimental Difficulties}

\subsubsection{Ferromagnetic Nanorods as a Nonlinear Beacon}

In order to generate a harmonic response, the nonlinear object must possess inherent nonlinearity in its material properties. Since TR uses electromagnetic waves, we are interested in possible nonlinear electric or magnetic properties. In this section, we discuss a potential magnetic nonlinear object in the form of ferromagnetic nanorods. These nanorods have a nonlinear magnetization curve. This means that the B-field generated from an interrogation signal should have a harmonic response that we may use for NLTR. We chose to use ferromagnetic nanorods specifically due to the potential medical application of hyperthermic treatment of tumors. The NLTR process may supply sufficient power needed to raise the temperature of the tumor, destroying it. Ferromagnetic nanorods may be injected into a tumor site as a nonlinear beacon, allowing this potential life-saving procedure to become a reality.

While the usage of diodes as nonlinear objects has been well-documented, the usage of ferromagnetic nanorods as nonlinear objects has not. As a result, we performed preliminary experiments on the ferromagnetic nanorods in order to determine whether or not the nonlinear response would be strong enough to successfully complete the NLTR process.

This preliminary experiment involved attaching ferromagnetic nanorods to an antenna (as shown in Figure 4-2), sending a pulse signal through that antenna, and then recording the response. In order to record the response, we used a spectrum 


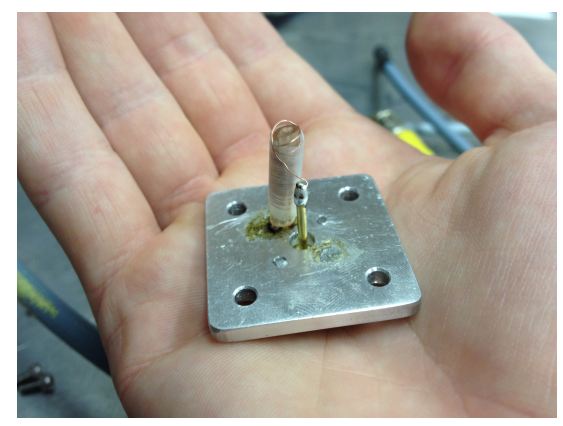

Figure 4-2: Antenna used for nanorod experiments. The nanorods are within the coil conntected to the monopole. The coil was used to maximize the magnetic field applied to the nanorods during use.

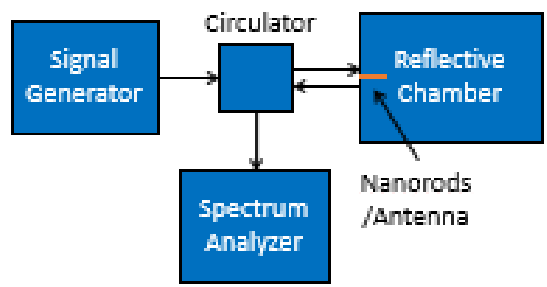

Figure 4-3: Nanorod experimental setup. The reflective chamber and equipment used is identical to that discussed in 3-1.

analyzer, as shown in Figure 4-3. By transforming the response signal to the frequency domain, we were able to easily identify whether or not a harmonic was generated.

Figure 4-4(a)-(d) show the $2 f$ response under a variety of conditions. Although there seem to be peaks in (a) - (d), the peaks are somewhat randomly distributed and are small in magnitude.

In these experiments, we expected and searched for a $2 f$ (second-order) harmonic response, because this has been observed in prior work with diodes. However, we realized later that ferromagnetic nanorods have been found to produce a noticable third-order (3f) harmonic repsonse [33]. As a result, we are unable to conclude from our experiments whether or not ferromagnetic nanorods would provide a sufficient nonlinear response for performing NLTR. Future work should conduct similar experiments, but focus on the $3 f$ frequency range instead. 


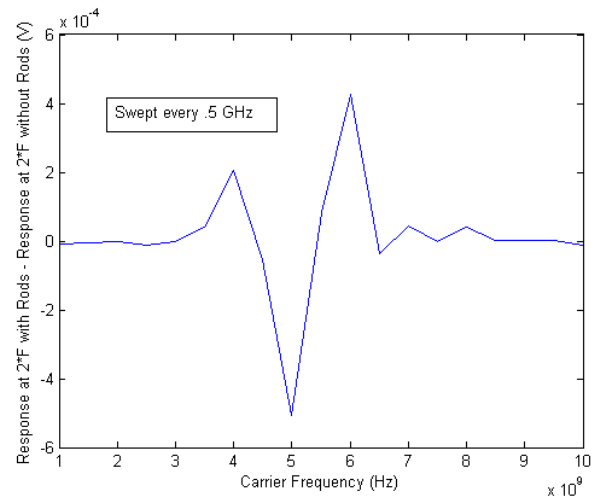

(a)

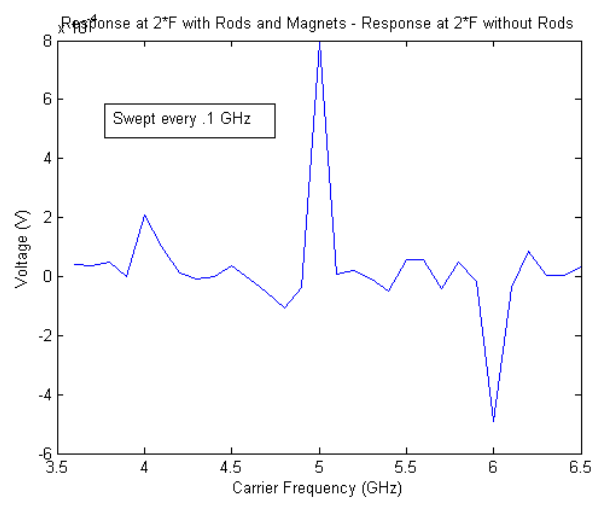

(c)

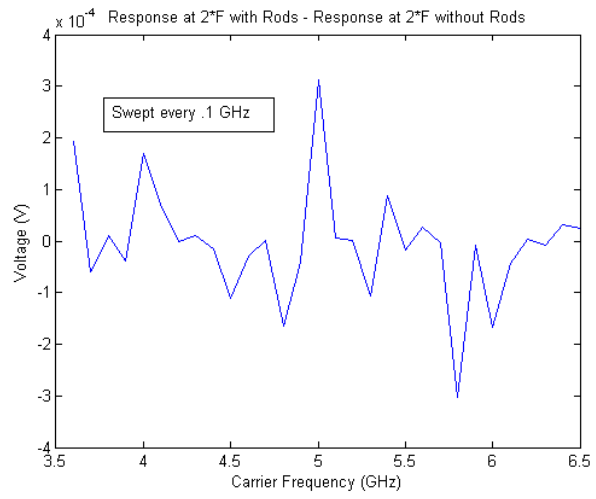

(b)

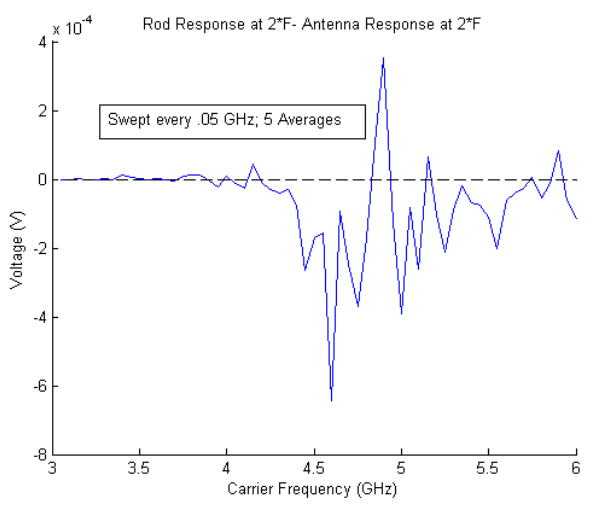

(d)

Figure 4-4: Experimental results. (a) - (d) show various ferromagnetic nanorod configurations' response spectra. No configuration leads to a significant $2 f$ response, as illustrated through the random and small amplitude response signals 


\subsubsection{Diodes as an Electric Nonlinear Beacon}

While utilizing a nonlinear magnetic response was not feasible, utilizing a nonlinear electric response has been well-documented through the use of a diode. This represents a nonlinear object, as the current response to an applied voltage for a diode changes significantly after a certain applied voltage value, as seen in Frazier et al. have previously used diodes in NLTR experiments to much success.

We performed another preliminary test to verify the nonlinear response of a diode after being interrogated by a Gaussian pulse. Given the history of diodes in NLTR, we believed that we would see a large $2 f$ response. While performing these preliminary tests we were unable to produce any measurable harmonic from a diode while it was present in the Gigabox. In order to circumvent this problem, we used extremely large amplifiers as done by Hong et al. in their NLTR experiments. We were still unable to generate a significant harmonic response.

As a last effort we used various frequency multipliers to generate a nonlinear signal. We did this to verify that the equipment used in our setup was functioning properly. We were able to extract a nonlinear sona from the frequency multiplier; however, this is impractical for WPT as frequency multipliers are large in size. This confirmed that our equipment were working properly, but that we were unable to generate a large

response. Since we were not able to generate a nonlinear sona experimentally, we conducted all of our NLTR experiments using numerical simulation. 


\subsection{Purpose}

Here, we focus entirely on numerical simulations of NLTR as they apply to WPT. Our focus was threefold: (1) to conduct simultaneous NLTR collapses onto two nonlinear objects, (2) to observe the selective collapse of NLTR onto two distinct nonlinear objects, and (3) to characterize the transmission efficiency of the process. From these three focuses, we demonstrated that the NLTR process may be generalized to an arbitrary number of nonlinear objects in an enclosure and that these reconstructions may be selectively rectified at the nonlinear objects. We provide a scheme to characterize the efficiency of our process but were unable to develop a conclusive theory for tuning the efficiency. Our results provide a baseline for developing an experimental algorithm for targeting objects in an enclosure for WPT applications, either selective or non-selectively. We will first detail the general scheme used for performing NLTR and follow with the setup, experimental results, and discussion for each of our three focus topics.

The process of developing a new technology required benchmarking each step diligently. Any successful WPT technology must have effective transmission efficiency, implying minimal loss in each step of the system. Given the broad frequency signals used in experimentation, a significant drawback to any TR experiment is the inherent wave reflection at interfaces between equipment, various mediums, or circuitry $[34,35]$. While wave reflection losses can be theoretically be measured via S Parameters (explained later) of an experiment, these sources of loss are not practically measurable due to the fact that measuring the losses would create addition interfaces 
in the system, introducing additional sources of wave reflection [34]. To calculate the true ceiling on effective transfer efficiency, it is necessary to numerically simulate the process, as a simulation can calculate the various losses without interfering with the system itself.

In addition to using simulations to study the sources of loss in our system, it also provided us with a simple nonlinear element: a model diode. As explained in previous chapters, the nonlinear response in our Gigabox experiments was found to be difficult to excite due to power limitations, noise, or equipment sensitivity. The difficulties that we encountered in our physical experimentation were circumvented by numerically simulating the NLTR process. By being able to monitor the voltage and current response of the diode at all times in the simulation, we were able to troubleshoot problems that occurred in the numerical NLTR process that would have otherwise have been impossible to determine in experimentation.

\subsection{Methodology}

\subsubsection{Equipment}

In order to perform these tests that were not possible in the Gigabox, we used the program Computer Simulation Technology: Microwave Studio (CST for short) to perform electromagnetic wave simulations. CST is an industry standard modeling program that uses the Finite Integration Technique (FIT) to numerically solve Maxwell's equations [36]. This technique is a generalized version of the well-known Finite Difference Time Domain (FDTD) method but can resolve complex geometries 
and boundary conditions in a simpler manner. We will not discuss these techniques at length, as they are well-understood in the literature [37,38]. Our team had two computers with a total of two shared CST licenses to perform simulations. The processing power, RAM, and GPU availability are shown below in Table 4.1. CPU A was used for either single simulations or computationally small simulations. A single simulation refers to a simulation where we did not sweep a parameter. CPU B was used for large simulations or parameter sweep simulations, requiring either large amounts of memory or large amounts of time, respectively. In general, we used CPU A to perform simultaneous and selective NLTR while we used CPU B to calculate transfer efficiency and characterize nonlinear response characteristics.

\begin{tabular}{|l|l|l|}
\hline & CPU A & CPU B \\
\hline Processor & $\begin{array}{c}\text { Intel Xeon CPU } \\
\text { X5670 @ 2.93 GHz }\end{array}$ & $\begin{array}{c}\text { Intel Xeon CPU } \\
\text { E5-2680 @ 2.50 GHz }\end{array}$ \\
\hline Number of Processors & 2 & 2 \\
\hline RAM & $44 \mathrm{~GB}$ & $128 \mathrm{~GB}$ \\
\hline GPU Available & No & Yes \\
\hline
\end{tabular}

Table 4.1: Technical specifications of the computers used for conducting all simulations and modeling.

\subsubsection{Time-Reversal and Nonlinear Sona Extraction}

To start any simulation, we first generated a Gaussian pulse signal in MATLAB. For this interrogation signal, we chose the amplitude, center frequency, and bandwidth 


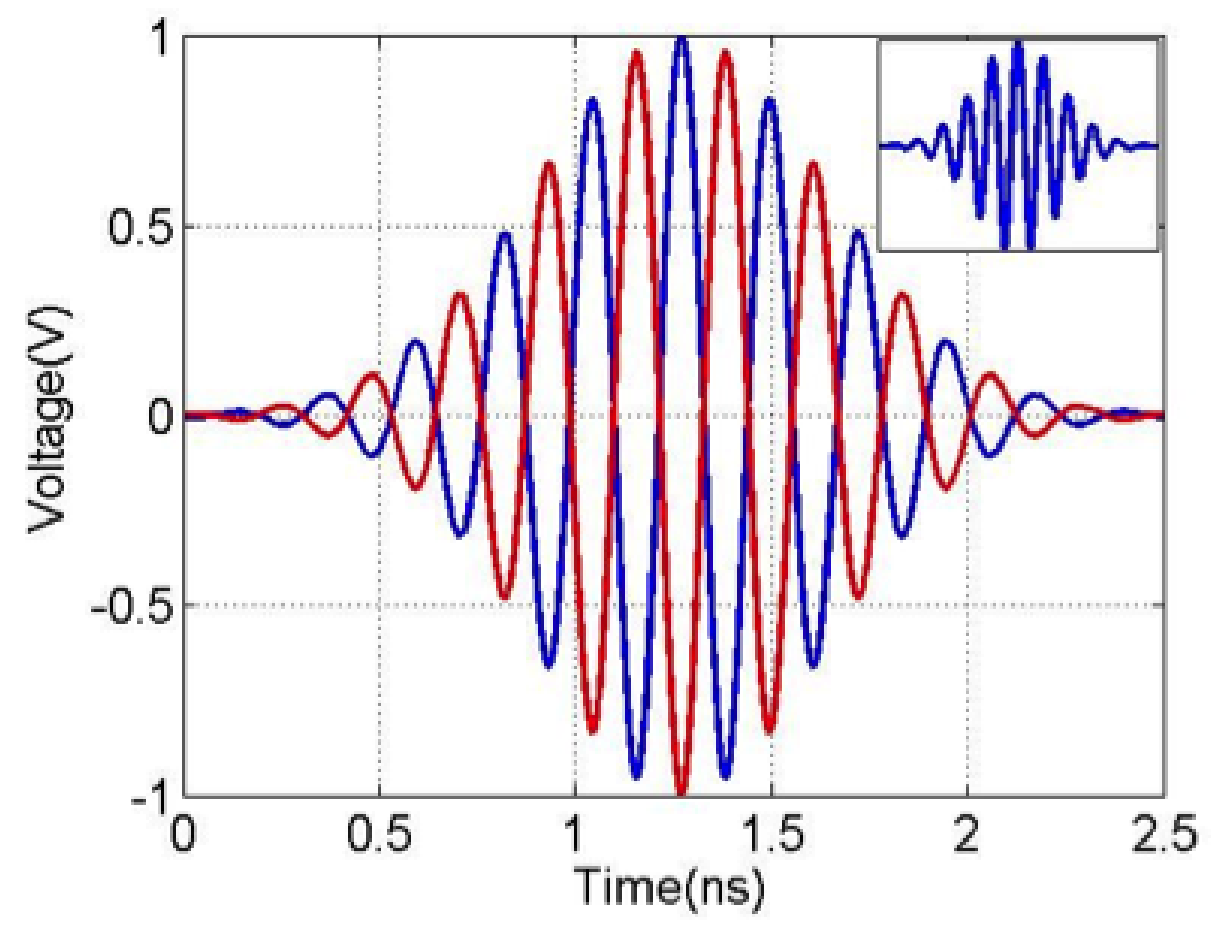

Figure 4-5: This shows our input waveform with a $4.4 \mathrm{GHz}$ center frequency and $1.0 \mathrm{GHz}$ bandwidth. The blue signal is the non-inverted input and the red signal is the inverted input. A singular, non-inverted waveform is shown in the inset.

frequency for our experiment. An example input signal is shown inset in Figure 4-5. The simulation was run using this pulse signal, noting one of the ports as an injection port (e.g. Port A) and one as the recording port (e.g. Port B).

In general, we had two methods to extract the nonlinear sona from our recorded signal: (1) applying a Fourier transform and band-pass filtering the raw signal and (2) pulse inversion. As previously discussed, many of the Gigabox experiments were performed by recording a sona, applying a Fast Fourier Transform (FFT), and finally using a band-pass filter on the recorded signals to extract the harmonic signal at the correct frequency. In our numerical simulations; however, the sonas were only recorded for 30-35 ns and the discretized nature of the data on this time scale led to 
distortion of the sona signals. The team also explored using pulse inversion, another method of sona extraction that has been well documented in the literature $[32,39]$. Hong et al. have used this method for numerous time-reversal experiments, citing its computational simplicity as a benefit for using it in physical experiments [32]. The process involves summing two sonas with inverted inputs. When the sonas are summed, linear signals are cancelled out, and nonlinear signals remain. Adding the sonas is significantly faster than applying a transformation to an entire sona signal, saving time. While this process proved to be very difficult to implement for our Gigabox experiments, it was quite efficient at producing the nonlinear sona in CST. Due to the simplicity of pulse inversion, we used this method for extracting all nonlinear sonas. In this method, we ran the same simulation once with our original input signal and then we ran it a second time with an inverted version of our original signal. Inversion simply means that the entire original signal was multiplied by -1 . Assuming a linear system, the sum of the original and inverted signals sums to 0; however, a nonlinear system sum is non-zero. Because the diode is the only nonlinear portion of the system, summing the original and inverted signals yields only the signal from the diode, which is the desired nonlinear sona. Figure 4-6(a) and (b) illustrate two sona signals, where the blue signal is the non-inverted sona and the red signal is the inverted sona. Figure 4-6(b) contains a nonlinear element while 4-6(a) does not. The corresponding (c) and (d) show the result of summing the two signals. It is clear that total annihilation of the sona signal occurs when no nonlinear element is present, as in 4-6(c), while a clear signal is present in 4-6(d).

This method to extract the nonlinear sona was chosen over taking the FFT and 

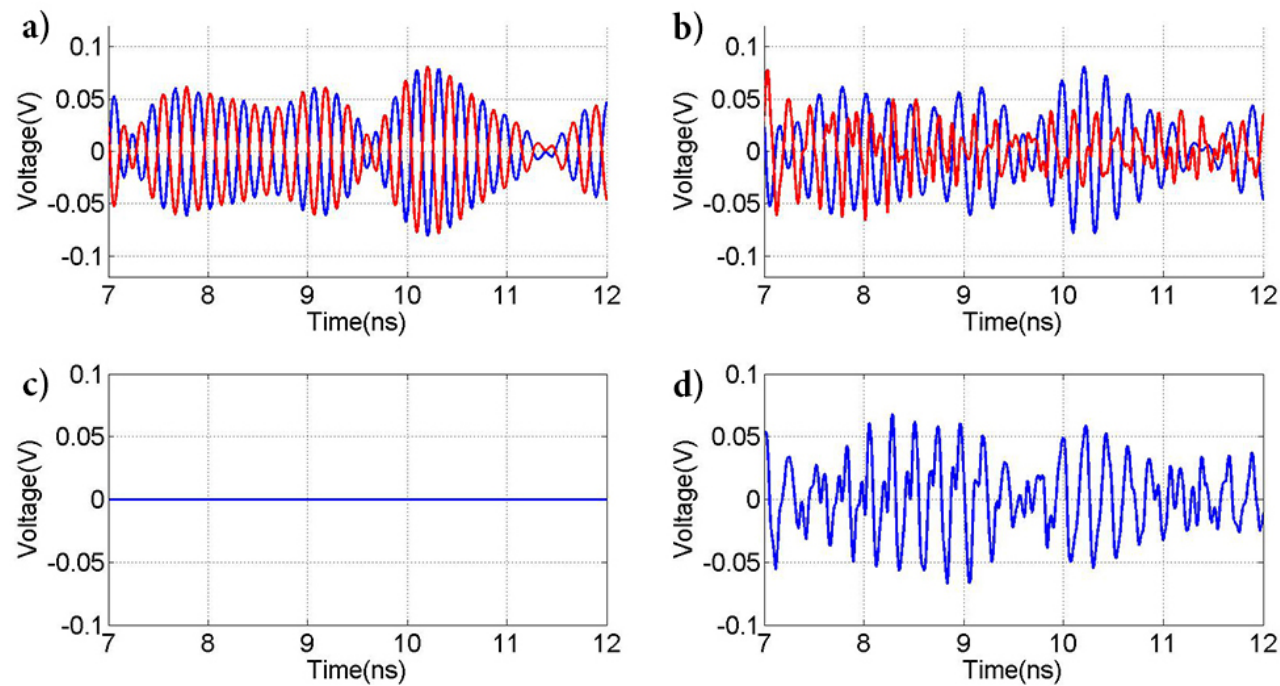

Figure 4-6: (a) shows a portion of the non-inverted (blue) and inverted (red) recorded response signal. (b) shows the same two response signals when a diode is present in the simulation. (c) the sum of the two signals in (a), resulting in signal annihilation. (d) the sum of the two signals in (b), which produce a non-zero signal.

filtering the recorded signal as sample rate for the simulation data was not high enough to ensure the results would not be distorted. This discretization of data caused the resulting time-reversed sonas to produce incorrect results using the FFT and filter method, while the time-reversed sonas produced correct results with the pulse inversion method.

\subsubsection{Defining and Controlling the Nonlinear Element}

In the simulations themselves, we used a model diode as our nonlinear object, as it has a nonlinear I-V curve. As previously stated, the diode represents a passive nonlinear object, as harmonics signals were generated as the diode was interrogated. This represents a truly passive WPT application. The diode location for each simulation was chosen semi-arbitrarily with the only restriction to not be within 1-2 wavelengths of either port, as this may have created near-field effects that influenced the results. 
In CST, the diode component is modeled as the following circuit (Figure 4-7) and mathematical relationship (Equation 4.1 and 4.2). From these, the user may change the parasitic capacitance $(C)$, the series resistance $(R)$, the reverse conductance $\left(G_{s}\right)$, and the functional temperature $(T)$.

For $V_{d}>0$ :

$$
I_{d}=I_{0}\left(e^{\frac{e V_{d}}{k T}-1}\right)=I_{0}\left(e^{\frac{V_{d}}{V_{k}} a-1}\right)
$$

For $V_{d}<0$ :

$$
I_{d}=G_{s} V_{d}
$$

To obtain idealized results, we set $C$ and $G_{s}$ to 0 , as these values reduced the magnitude of the nonlinear response. We used the default $R=50 \Omega$, as very large $R$ reduced the overall signal greatly and a very small $R$ did not produce any harmonics. This left only $T$ to tune the I-V curve of the diode. In real life, different diodes have different I-V curves based on material. In order to simulate these differences mathematically, we changed the temperature of the diode in the simulation, as shown in Figure 4-8.

In reality, this changed the knee voltage $\left(V_{k}\right)$ for the diode. We chose $V_{k}$ to be the applied voltage needed to achieve a current of $0.2 I_{0}$. The parameter $a$ is used to define this $0.2 I_{0}$ cutoff. As discussed later in this chapter, we will show how the nonlinear response of a diode is dependent on both the input pulse amplitude and the knee voltage. This nonlinear response was maximized to allow for selective targeting between two diodes simultaneously. 


\subsection{Results}

\subsubsection{Simultaneous Nonlinear Time Reversal}

The first focus of our numerical simulations was to illustrate the collapse of a nonlinear time-reversed sona on multiple targets simultaneously. To be an effective WPT system, our technology would need to be able to charge more than one device at a time. Based on literature illustrating NLTR collapsing on a single nonlinear object, we hypothesized that a nonlinear sona would collapse on an arbitrary number of nonlinear objects if the nonlinear objects were present in the time-forward step of NLTR $[4,40]$. We assumed that the reconstruction on each nonlinear object would sum linearly and independently such that no reconstruction would interfere with another, as shown in Equation 4.3, where $R_{i}$ is the reconstruction on a single nonlinear object and $x_{i}$ is a weighting of the amount of energy that the single nonlinear object contributes to the overall energy of all reconstructions. Based on this, we expected the reconstruction waveforms of the independent single-diode simulations to match a single, multi-diode simulation.

$$
R_{t o t}=\sum_{i} R_{i} x_{i}
$$

This feature was realized by creating a geometry with two diodes present along with two ports to record and emit signals, shown below. We created a quasi-twodimensional (2D) irregular cavity in CST. This cavity was a $15 \mathrm{~cm}$ x $15 \mathrm{~cm}$ x $0.76 \mathrm{~cm}$ square box modified with various circular and elliptical segments removed from the walls as shown in Figure 4-9. The $0.76 \mathrm{~cm}$ height was chosen to maintain a 2D simu- 

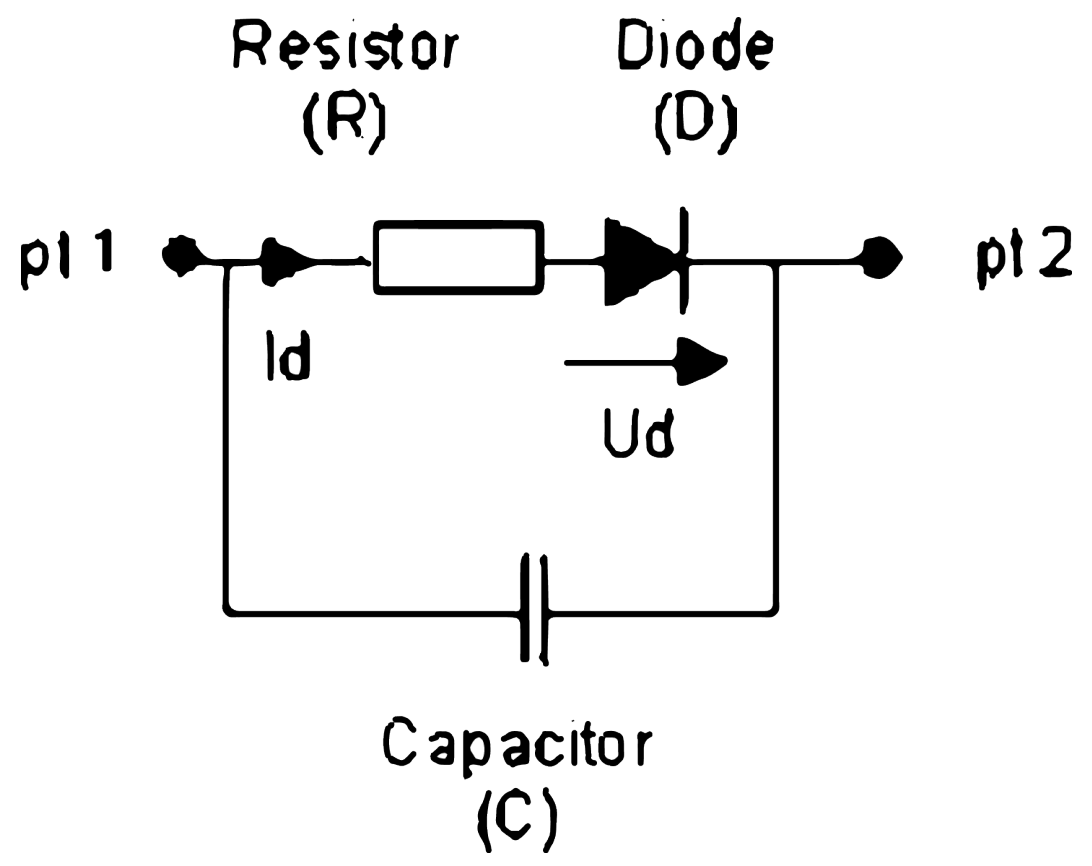

Figure 4-7: CST diode circuit model

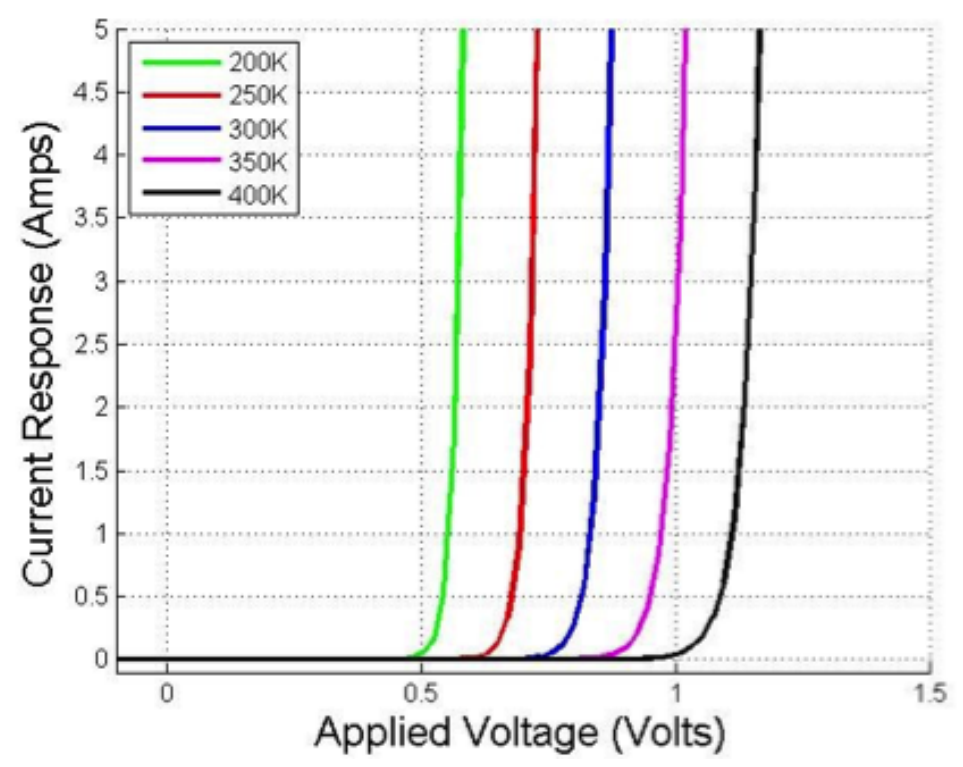

Figure 4-8: Different I-V curves that can be modeled in CST. It should be noted that the "Temperature" of the diode only changes its mathematical definition of the I-V curve 

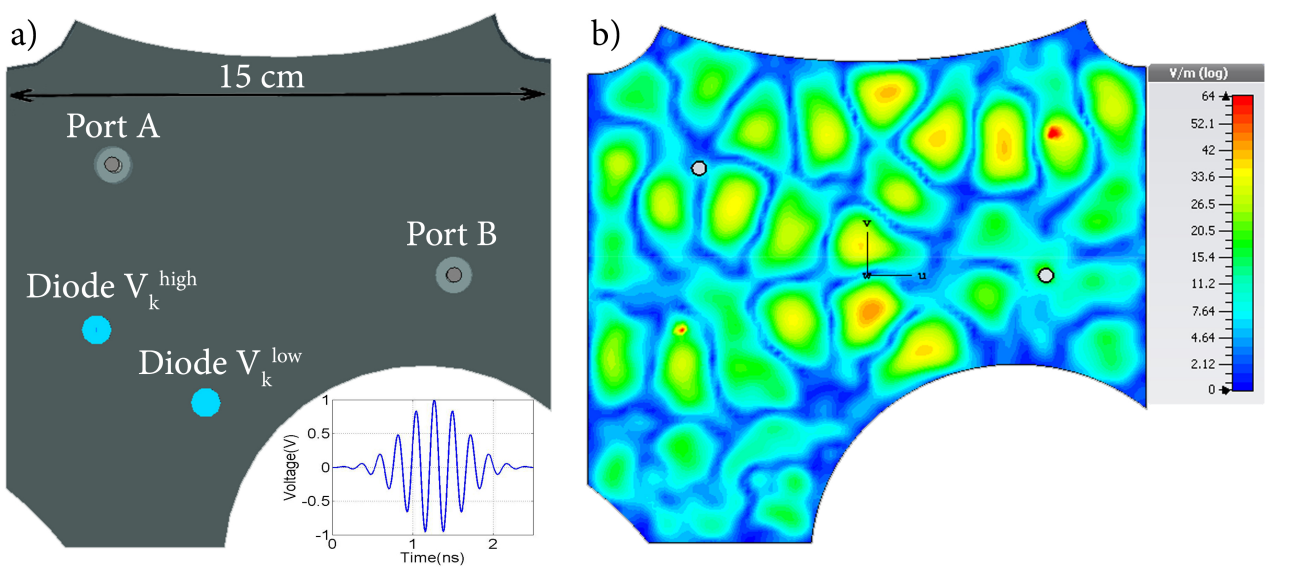

Figure 4-9: The Cut Box simulation geometry used in CST. (a) shows the location of the 2 ports (A and B), 2 diodes $\left(V_{k}^{\text {high }}, V_{k}^{\text {low }}\right)$, length scale, and the lower inset shows the initial interrogation pulse. (b) shows the electric field at a point in the simulation, illustrating the limited excited mode density.

lation. Equation 4.4 was used to calculate the cutoff frequency for the fundamental mode of a parallel plate cavity, below which only 1 frequency will propagate, reducing the time needed to simulate [35].

$$
f_{c}=\frac{c}{2 h}
$$

Using a cavity of height $0.76 \mathrm{~cm}$ resulted in a cutoff frequency of $20 \mathrm{GHz}$, well above our typical test frequencies of $4-5 \mathrm{GHz}$ fundamental and 8-10 $\mathrm{GHz}$ harmonic. We chose this cutoff frequency in the event we needed to use a higher fundamental frequency to create better spatial resolution of our reconstruction. The area dimensions for the Cut Box Model were chosen to minimize computational time while still maintaining a reasonable mode density, as shown in Figure 4-9(a). This mode density is required to allow the ray chaotic environment to have high sensitivity to initial and boundary conditions; a low mode density will prevent NLTR from having high spatial resolution. 


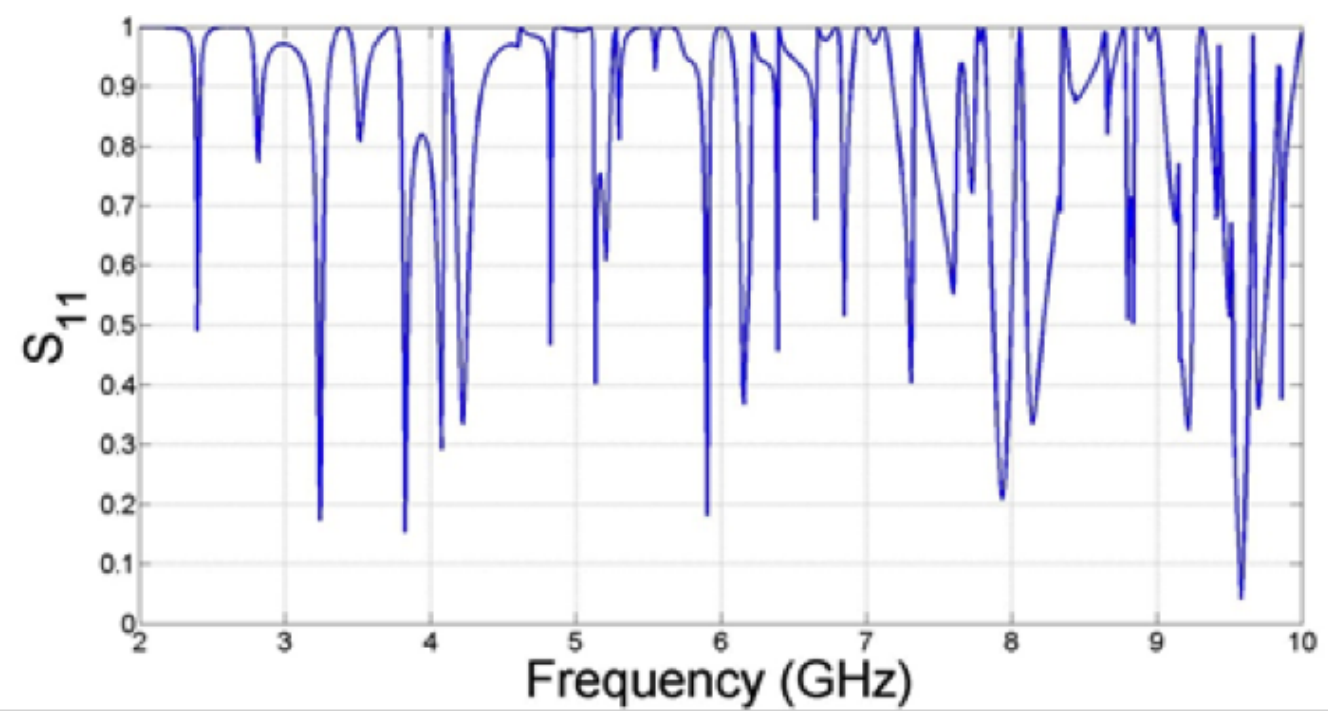

Figure 4-10: The $S_{11}$ spectrum for the Cut Box Model. We wanted to minimize $S_{11}$ for our simulation while maintaining a large bandwidth. This led to choosing a $4 \mathrm{GHz}$ center frequency with a $1.0 \mathrm{GHz}$ bandwidth.

We used two Teflon-coated dipole antennas to emit and record signals [41]. Two diodes are placed inside the cavity, shown by the blue circles in Figure 4-9. A $4.4 \mathrm{GHz}$ center frequency pulse with a 1.0 GHz bandwidth was chosen to minimize the reflected power and applied to antenna A. The interrogating pulse is shown as an inset in Figure 4-9(a).

The specific frequency and bandwidth was chosen for this geometry to minimize initial wave reflection $\left(S_{11}\right)$ into the enclosure. Figure $4-10$ shows that using a $1.0 \mathrm{GHz}$ bandwidth, an optimal center frequency is $4.4 \mathrm{GHz}$. The dips in the $S_{11}$ value represent optimal frequencies to use, as a low $S_{11}$ indicates a large portion of the signal entered the cavity. The large number of peaks is indicative of the mode density of the geometry, where low frequencies are sparse given the relatively small scale of the cavity.

Using this input pulse and the pulse inversion method of sona extraction, we were 
able to perform NLTR in the Cut Box Model. By measuring the observed voltage and corresponding current during the time-reversed step, we measured the instantaneous power over time in each diode. As shown in Figure 4-11, the reconstruction waveform on each diode matches the expected results for a 1-diode experiment $[27,29]$. We also measured the instantanous power at a non-target location near Port A, shown in green in Figure 4-11. The ratio of the the peak-to-peak instantaneous power for each target diode to non-target diode is 10.1:1 and 7.84:1 for Diode 1 and Diode 2, respectively. This also corresponds to an integrated power ratio of 139.7:1 and 56.4:1 for Diode 1 and Diode 2, respectively, compared to the non-target integrated power. Here, we define integrated power as the total deposited energy during the time reversed step, as shown in Equation 4.5

$$
E=\int_{0}^{t_{f i n a l}} I(t) V(t) d t
$$

\subsubsection{Discussion of Simultaneous Nonlinear Reconstructions}

This result alone signifies the generality of NLTR whereby any nonlinear object in the cavity will observe a reconstruction in the time-reversed step. In regards to a larger WPT system, one can imagine that this is particularly useful inside of a home or public area, where any device may be powered as long as it is within range of the transmitter/receiver base station. Because the process used only creates a reconstruction at the nonlinear element, it is as simple as repeating the NLTR process rapidly, creating a quasi-pulse width modulation (PWM) signal that can charge a battery. PWM is a method for controlling how active a device is by rapidly 


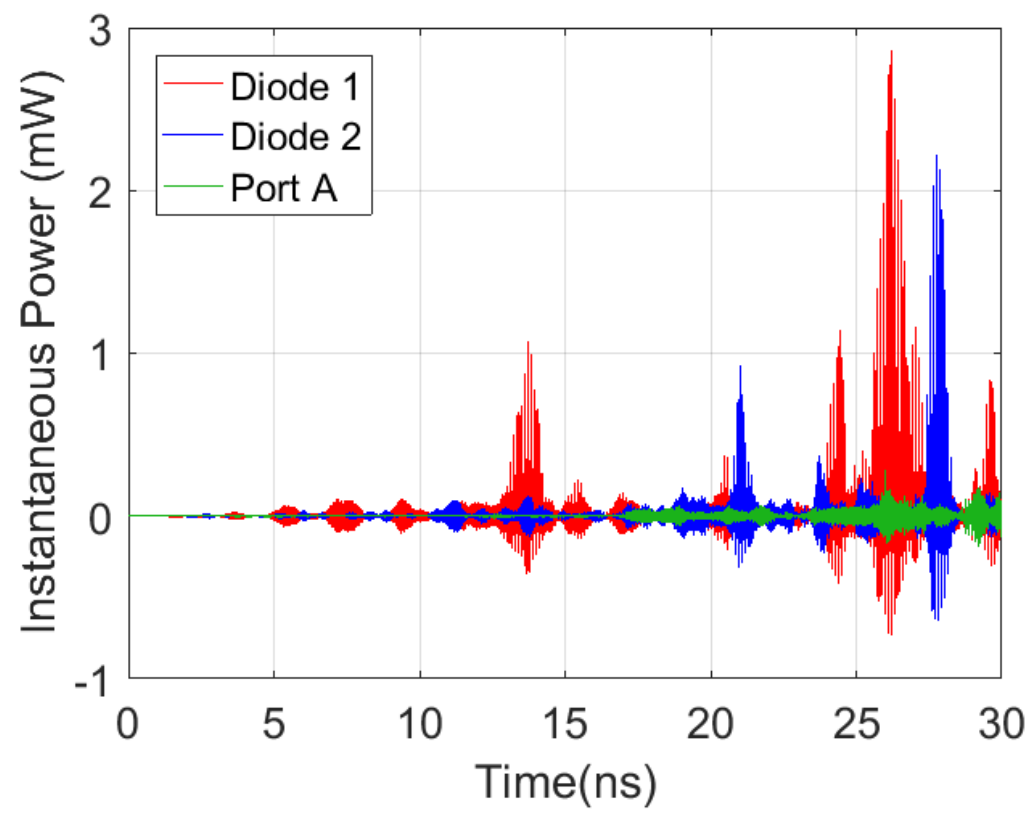

Figure 4-11: The time-reversed reconstruction on two diodes simultaneously performed inside the Cut Box geometry.

switching the device on and off. For example, a fan has different speeds because it operates $25 \%, 50 \%, 75 \%$, or $100 \%$ of the time. Similarly, if we can reconstruct on a battery circuit every $10 \%$ or $20 \%$ of the time, we can effectively charge the battery. As soon as the nonlinear object leaves the enclosure, it will no longer receive power. By having a base station that can actively modulate output sona power, it would be very simple to change the amount of energy transferred to each device. This dynamic power control is outside the scope of our project but represents a much later extension to this WPT system.

\subsubsection{Simulation of Selective Collapse of NLTR}

The logical next step is to determine how such a system would be able to determine which device should be powered. As previously shown, if nothing is done to alter the 
sona signals, then the sona from each nonlinear object will sum linearly and produce a reconstruction at all nonlinear objects in the time-reversed step. Given that each reconstruction sums independent of one another, we hypothesize that we should be able to eliminate the contribution of one nonlinear reconstruction to the overall set of reconstructions without altering the fidelity of any other individual reconstruction on other nonlinear elements. For example, in an enclosure with 3 diodes, if we could suppress the response of diode 2 in the time-forward step, then we would expect to only observe a reconstruction on diodes 1 and 3, creating a selective targeting method. Using Eq. 3, this would imply a weight of $x_{2}=0$, as shown in Equation 4.6.

$$
R_{t o t}=\sum_{i} R_{i} x_{i}=R_{1} x_{1}+R_{2} * 0+R_{3} x_{3}=R_{1} x_{1}+R_{3} x_{3}
$$

In a commercial setting, this would be an extremely useful aspect to our WPT system, as companies could require payment to use the system and would otherwise suppress the reconstruction on the user's device. The fact that the nonlinear object is passive in the environment implies that this selective targeting could even be used to "resurrect" a dead phone, a feature not seen on any current WPT technology on the market.

We have developed a method of performing such a targeting scheme based on the previously mentioned diode model. In the simplest case, we show that selective targeting for 2 diodes with two separate voltage knees was obtained (noted $V_{k}^{h i g h}$ and $V_{k}^{\text {low }) .}$

Recall the current response in the diode from Equation 4.1. One useful aspect to the diode definition function is that if $V<V_{k}$, then the diode has very little current 


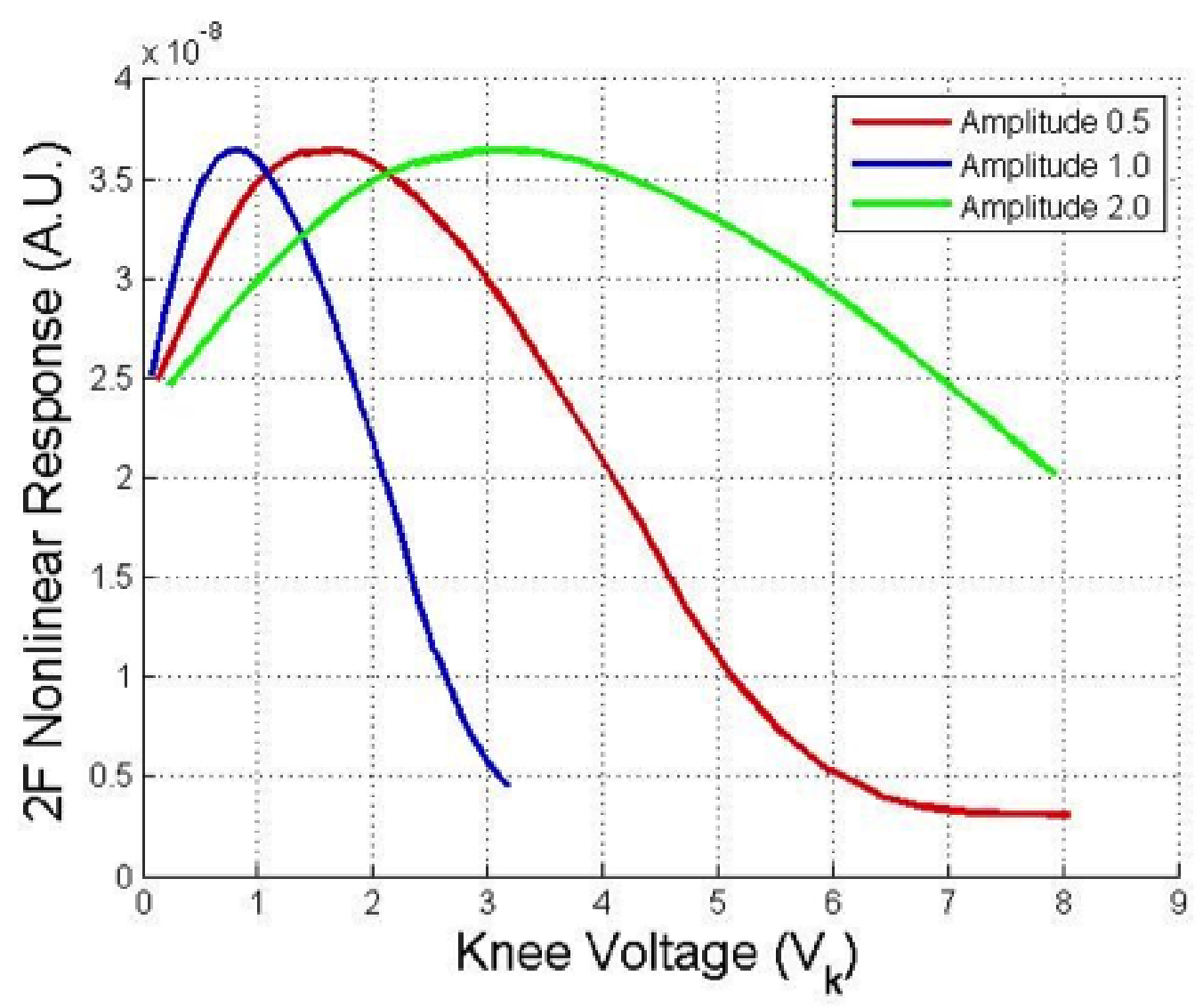

Figure 4-12: The nonlinear response of the diode had a clear maximum at a specific knee voltage. By using a pulse amplitude that corresponded with the specific diode knee voltage, we could selectively target diodes in CST.

response, which in turn implies a low nonlinear response. We utilize this characteristic while performing selective targeting on a low $V_{k}$ diode. Similarly, we observe that at small $V_{k}$ values, the nonlinear response is small. These two observations led to the hypothesis that nonlinear response may be maximized given an initial pulse by selecting a proper $V_{k}$ value. As shown below, Figure 4-12 was obtained by sweeping over many $V_{k}$ values for different initial pulse amplitudes given the same geometry and process conditions as previously stated. Given this distribution, we may target a high $V_{k}$ diode, as the nonlinear response will be stronger in the high $V_{k}$ diode than the low $V_{k}$ diode. 
To perform this selective targeting, we once again used the Cut Box Model. In the simplest case, we consider selective targeting of a low $V_{k}=0.79 \mathrm{~V}\left(V_{K}^{\text {low }}\right)$ diode at the exclusion of a high $V_{k}=6.60 \mathrm{~V}\left(V_{k}^{\text {high }}\right)$ diode. Given the large value of $V_{k}^{\text {high }}$, we expect to see no response in that diode while maintaining a reasonable nonlinear response in the $V_{k}^{\text {low }}$ diode. We used a pulse with amplitude $V=1.0 \mathrm{~V}$ during the time forward step, emitted at Port A in Figure 4-9(a). Using pulse-inversion, we extracted the nonlinear sona from the recorded signal at Port B, time-reversed the signal, and re-emitted it from Port B. Figure 4-13 shows the time-reversed reconstructions on both diodes. This results in a reconstruction almost exclusively on the $V_{k}^{\text {low }}$ diode during the time-reversed step. To calculate the quality of the selective reconstruction, we determined the aspect ratio by comparing the max of $I V$ on both diodes as well as the integrated power on both diodes. For this scenario, we calculate a power delivery aspect ratio of 10.1:1 for the $V_{k}^{\text {low }}$ diode relative to the diode with $V_{k}^{\text {high }}$ and an integrated power ratio of 402:1. This illustrates our ability to deliver substantially more energy to the $V_{k}^{\text {low }}$ diode than the the $V_{k}^{\text {high }}$ diode while keeping the reconstruction compact.

The more difficult scenario was to selectively target a high $V_{k}$ diode at the exclusion of a low $V_{k}$ diode. We utilized the peak in nonlinear response as a function of knee voltage from Figure 4-12. By choosing the correct pulse amplitude corresponding to a peak value that was matched to the high $V_{k}$ value, we expected to see the strongest nonlinear response, resulting in the largest magnitude reconstruction. In this scenario, the low $V_{k}$ diode still observed a reconstruction; however, it was smaller in amplitude than the high $V_{k}$ diode reconstruction. Given that a real system would involve a full 


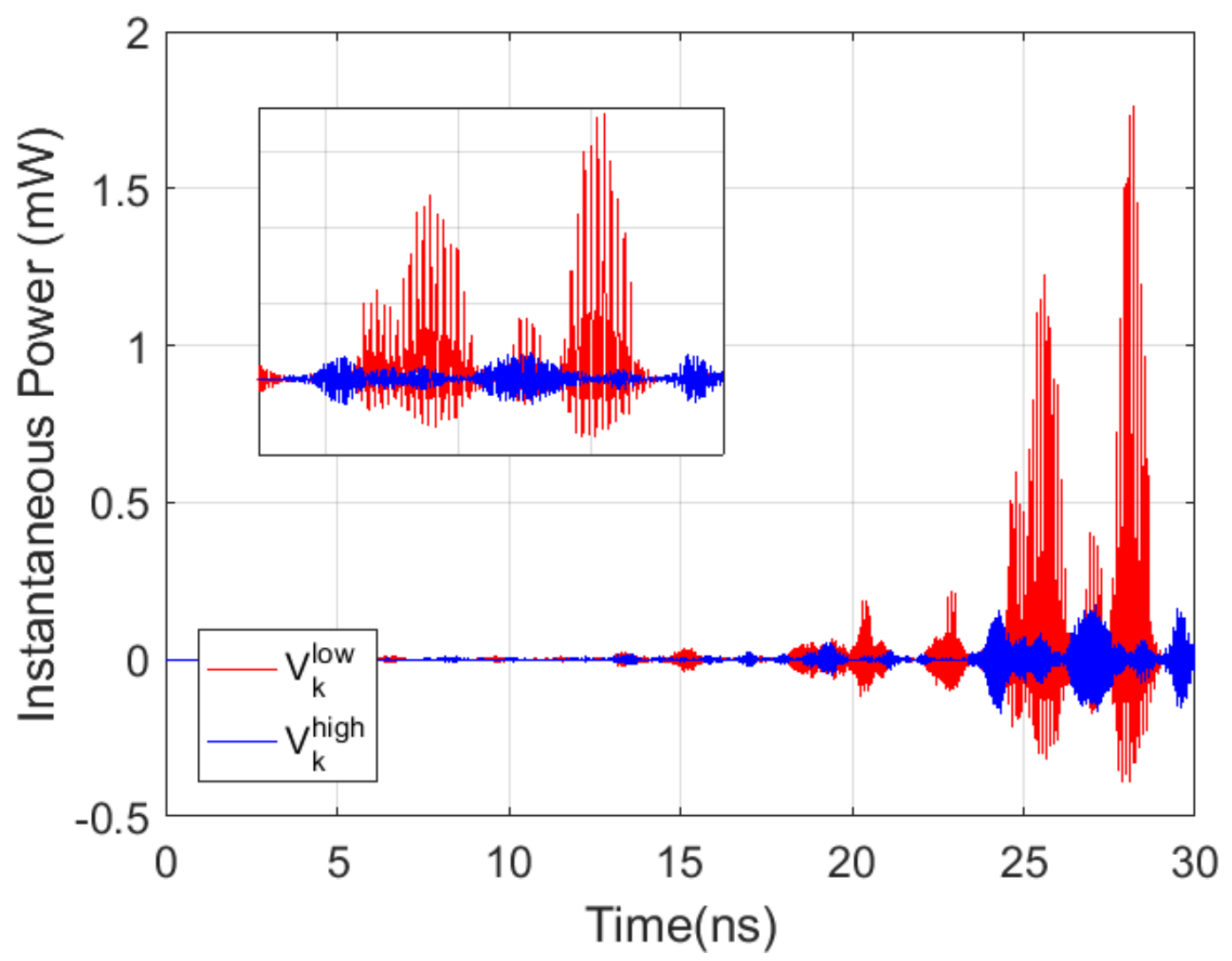

Figure 4-13: Time-reversed reconstructions on the two diodes while targeting $V_{k}^{\text {low }}$. Very little signal is seen on the $V_{k}^{\text {high }}$ diode. The insert shows the details of the reconstructions between 22 and $30 \mathrm{~ns}$.

rectification circuit in addition to the diode, the smaller amplitude reconstruction may not be able to turn on the rectifier while the high amplitude reconstruction at the high $V_{k}$ diode would turn on. For our simulations, we were able to model this by measuring the observed power $(I * V)$ on the diode and modify the sona amplitude in order to ensure the $V_{k}^{\text {high }}$ diode had rectification while the $V_{k}^{\text {low }}$ diode did not.

For this second scenario, with $V_{k}^{h i g h}=2.22 \mathrm{~V}$, we once again used an amplitude $1.0 \mathrm{~V}$ pulse during the time-forward step so that we may compare the results from both scenarios equally. As shown in Figure 4-14, pulse amplitude of $1.0 \mathrm{~V}$ has a maximum nonlinear response at $2.22 \mathrm{~V}$. We chose this $V_{k}$ value as it gave the clearest 


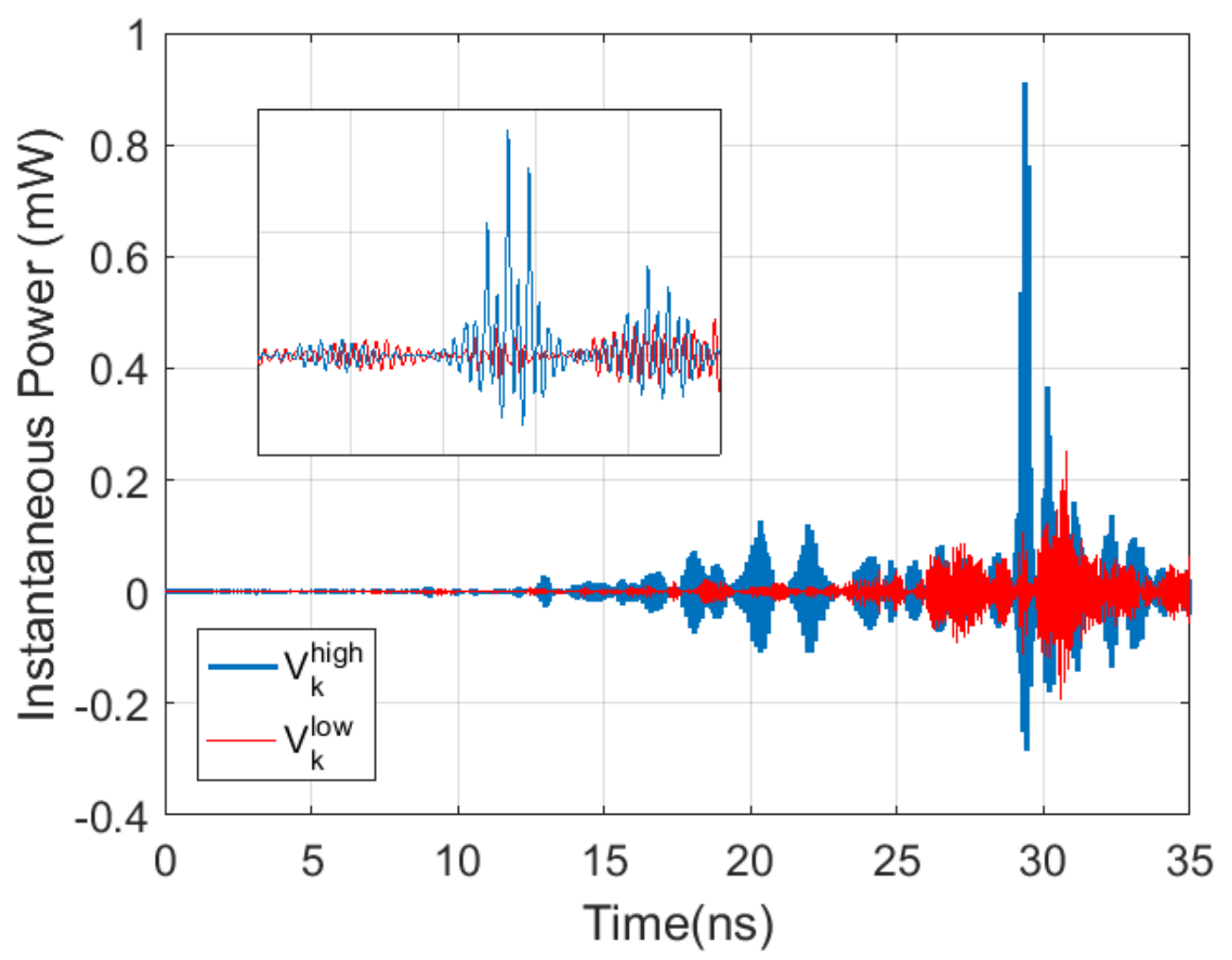

Figure 4-14: Time-reversed reconstructions on the two diodes while targeting $V_{k}^{h i g h}$. Sona amplitude modulation resulted in a clear contrast between the two signals.

reconstruction. Once again, we used pulse-inversion to extract the nonlinear sona. This resulted in selectively reconstructing on $V_{k}^{\text {high }}$ only during the time-reversed step with an aspect ratio of 3.61:1 for the $V_{k}^{\text {high }}$ diode relative to $V_{k}^{\text {low }}$, as shown in Figure 414. Comparing the integrated power for both diodes resulted in ratio of $6.75: 1$ for the energy in the $V_{k}^{\text {high }}$ diode relative to the $V_{k}^{\text {low }}$. The reduction in integrated power ratio can be attributed to the very short reconstruction around $29 \mathrm{~ns}$, which we believe is an artifact of modifying the sona amplitude to suppress rectification in the $V_{k}^{\text {low }}$ diode. Still, the energy is compact in the reconstruction signal as expected. 


\subsubsection{Discussion of Selective Nonlinear Time Reversal}

We have demonstrated a basic method for creating selective rectification using NLTR to target different nonlinear objects. This represents a stepping stone from the previously shown method of generalizing NLTR. By altering the input pulse amplitude, we were able to achieve the hypothesized nonlinear response suppression of specific nonlinear elements. This shows that the nonlinear reconstruction created on each nonlinear element is independent of one another and may be modified without impacting the other elements of the overall reconstructions. We have shown that this WPT technology would be capable of ubiquitous charging on any nonlinear device as well as selective targeting of specific devices. One may think of this as having an NLTR charger in one's home compared to having an NLTR in a business. In a domestic setting, there is very little need to restrict access to power. There are many outlets in one's home and there is no need to tell someone they are not permitted to use it. In a business however, a power outlet is not available to customers unless they pay for it. In this case, it is very useful to be able to selectively choose who may receive power. Although we have not shown a full-fledged system to do so, we have presented a foundational process that may be improved upon in order to create a successful NLTR-based WPT system.

\subsubsection{Simulation of Transmission Efficiency of NLTR Process}

We have so far shown that NLTR may be used in a WPT system to either power all nonlinear objects or selectively target the nonlinear objects within a relevant range. 
One significant question though that arises is to what efficiency are these objects powered. Surely a system that can only transfer $2 \%$ of applied power will not be a successful WPT technology, as the consumer is paying for the luxury to not carry around a cable. If the user has to pay 50x more for the energy they are very unlikely to use our system. As previously discussed, energy losses are often difficult to calculate in real experiments due to wave reflection and other systematic sources of error. However, by simulating the NLTR process, we are able to observe approximate transfer efficiency. In CST, we are able to track input power and power loss via wave reflection, absorption in both lumped elements and environment materials, and radiation loss at other ports. One drawback to using the built-in CST power tracking is that everything is recorded in the frequency domain rather than time domain. For example, a typical power spectrum from a time-reversed step of a 2-diode NLTR simulation is shown in Figure 4-15. As we are interested in overall power input compared to overall power output, we used this data along with Equation 4.7 to compute the efficiency of transfer in our simulations.

$$
e=\frac{\sum_{i} P_{i(\text { diode })}(f)}{\sum_{i} P_{i(\text { input })}(f)} \cdot 100
$$

The above data was taken from a simulation in the Cut Box model for the previously discussed two-diode simultaneous reconstruction. For this simulation, we found that $18.6 \%$ and $7.3 \%$ of power were transferred from the nonlinear sona to diodes 1 and 2, respectively. This provides a promising outlook.

Our next question was whether or not these transfer efficiencies were a function of distance between transmitter port, diode, and receiver port. We found that using 


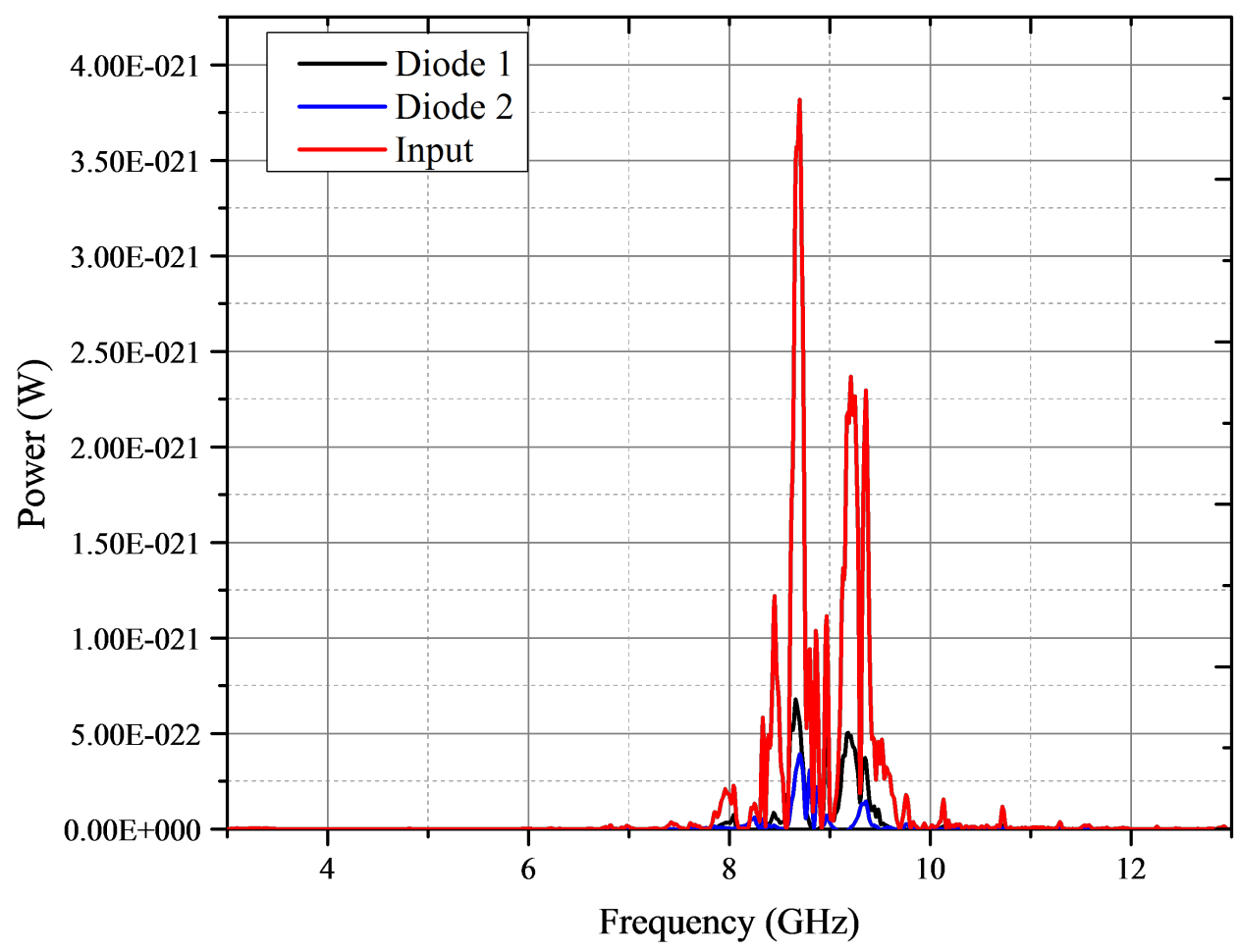

Figure 4-15: A typical power spectrum obtained in the time-reversed step (reconstruction). The power that enters the diodes only represents a portion of the overall power input in the simulation.

similar diode positions as previously stated resulted in transmission values around the $7-18 \%$ shown above. We decided to introduce a new model with a much larger length scale, called the Room Model shown in Figure 4-16.

We chose to use a $45 \mathrm{~cm}$ x $45 \mathrm{~cm}$ x $0.76 \mathrm{~cm}$ initial box to simulate a large geometry while still maintaining a quasi-2D cavity. The $45 \mathrm{~cm}$ length scale was used, as any larger simulation ran out of memory prior to completion. Due to the length of simulation and decreased nonlinear signal strength, this model was unable to be used for further efficiency calculations. From Figure 4-16, the distances in the simulation are much larger than the Cut Box and may be used in the future to determine whether 

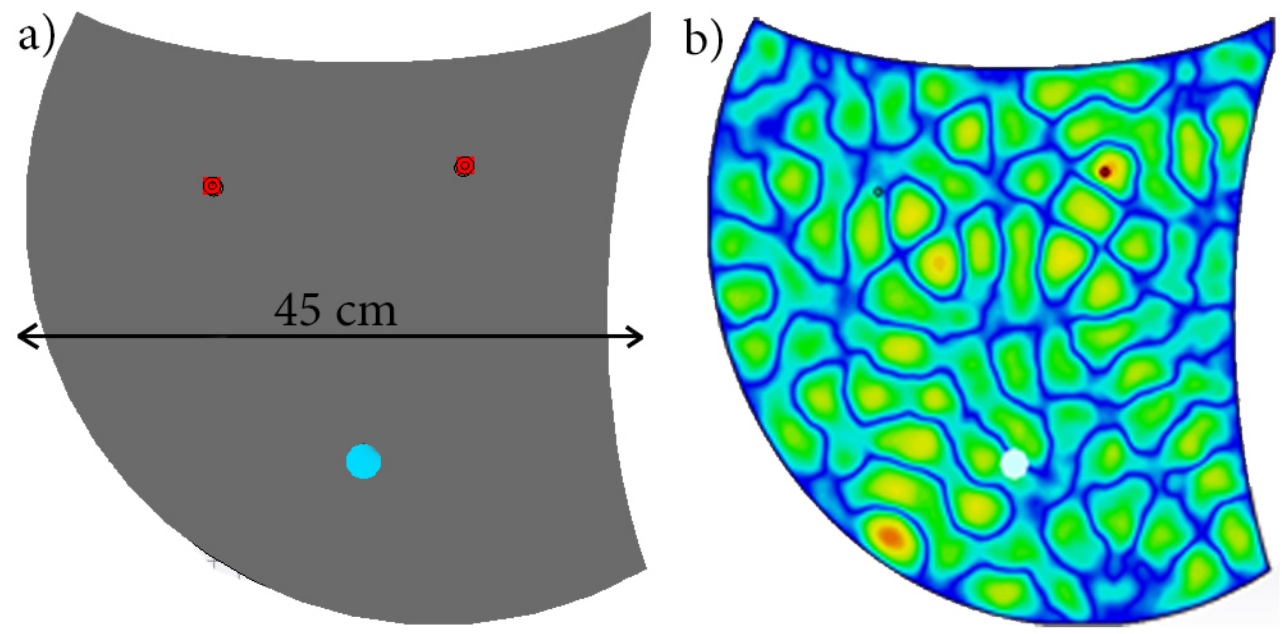

Figure 4-16: (a) illustrates the geometry of the Room Model and (b) shows the mode density of the space.

transmission efficiency of NLTR is dependent on distance between transmitter, nonlinear object, and receiver ports.

\subsection{Summary}

Using CST, we have successfully demonstrated our 3 goals: (1) simultaneous reconstructions on multiple nonlinear objects (2) selective reconstructions between multiple nonlinear objects and (3) calculation of a baseline transmission efficiency of NLTR. For simultaneous and selective targeting, we provide a basic framework to perform these processes using numerical simulations; however, these processes are still yet to be performed in real-world experimentation. We believe that this illustrates a foundational work for future experiments to demonstrate the feasibility of these features in a new WPT technology. 


\section{Chapter 5: Development of a Super Rectenna}

\subsection{Goals of Rectenna}

One of the main components of any WPT system is its rectifier, as it performs the crucial step of capturing the broadcast energy and transforming it into a usable form. To accomplish this, a rectenna, a dual-purpose device that combines an antenna and a rectifier, is necessary. The antenna picks up the oscillating AC signal received from the transmitter, the rectifier converts the signal to relatively stable DC power, and this usable power is passed to an arbitrary load.

In addition to standard rectification, the rectenna must serve as a passive nonlinear element. In NLTR, a nonlinear element is required to produce harmonics from the initial interrogation pulse broadcast by the transmitter. This goal is secondary to the main purpose of rectification.

The rectenna was designed to satisfy the following parameters. First, the rectenna must operate efficiently at the high frequency $(1-10 \mathrm{GHz})$ required for efficient NLTR. Additionally, losses due to reflection and parasitic effects within the antenna should be minimized. Finally, to fulfill the role of a nonlinear element, the rectenna should generate distinguishable harmonics strong enough for the purposes of NLTR.

In short, to rectify and generate harmonics for NLTR, the rectenna must be capable of not only efficient rectification at high frequencies, but also production of strong 
harmonics for NLTR.

\subsection{Diode Selection and Testing}

The diode is the most important single component to consider for the rectenna, as it is the main rectifying component. Careful consideration of its characteristics is vital. The switching frequency, forward voltage drop, and impedance of the diode are of particular importance to the desired goal of efficient rectification.

For the rectenna in our design to perform efficiently, the diode must satisfy several requirements:

The diode must maintain rectification functionality at a transmission frequency of 1-10 GHz. This requirement severely limits the possible choices for the rectenna diode. The frequency of a diode is inversely related to its reverse-recovery time $\left(t_{r r}\right)$, the fastest time the diode can switch from forward to reverse bias [42]. In traditional $\mathrm{P}-\mathrm{N}$ junction diodes, minimum $t_{r r}$ is on the order of tens to thousands of nanoseconds, even for fast diodes [43]. In a high frequency circuit, a P-N diode would be no different than a short circuit, and no rectification would occur.

The diode must have the lowest forward voltage drop possible. The voltage drop represents the reverse bias that remains when the diode works in the forward direction, and can be a significant contributor to power loss. The team's experimental WPT capabilities are limited to fairly low voltage microwave signals $(.5 \mathrm{~V}$ to $1 \mathrm{~V}$ signal amplitude), which makes forward voltage drop especially important.

Finally, impedances should be minimized. All diodes have intrinsic impedances 
such as parasitic capacitance and resistance. Impedances contribute to losses during rectification, and minimizing these losses would increase the efficiency of rectification.

Considering these factors, a Schottky barrier diode was chosen for the rectenna. Unlike P-N junction diodes, Schottky diodes have extremely low $t_{r r}$ and low forward voltage [43]. Schottky diodes do have a lower maximum reverse voltage rating, higher reverse leakage current, and higher cost than P-N diodes. However, these are relatively minor issues in our application. A Schottky MA4E1317 diode was used as the rectifying diode. The diode parameters from the manufacturer's datasheet are shown in Table 5.1.

\begin{tabular}{|c|c|c|c|c|c|}
\hline Test Conditions & Symbol & Units & Min. & Typ. & Max. \\
\hline Junction Capacitance at $0 \mathrm{~V}$ at $1 \mathrm{MHz}$ & $\mathrm{Cj}$ & $\mathrm{pF}$ & - & .020 & - \\
\hline Total Capacitance at $0 \mathrm{~V}$ at $1 \mathrm{MHz}$ & $\mathrm{Ct}$ & $\mathrm{pF}$ & .030 & .045 & .060 \\
\hline Junction Capacitance Difference & $\mathrm{DCj}$ & $\mathrm{pF}$ & - & - & - \\
\hline Series Resistance at $+10 m A$ & $\mathrm{Rs}$ & $\Omega$ & - & 4 & 7 \\
\hline Forward Voltage at $+1 m A$ & $V f_{1}$ & $\mathrm{~V}$ & .60 & .70 & .80 \\
\hline Forward Voltage Difference at $+1 m A$ & $\mathrm{DVf}$ & $\mathrm{V}$ & - & - & - \\
\hline Reverse Breakdown Voltage at $-10 \mu A$ & $\mathrm{Vbr}$ & $\mathrm{V}$ & 4.5 & 7 & - \\
\hline SSB Noise Figure & $\mathrm{NF}$ & $\mathrm{dB}$ & - & $6.5^{4}$ & - \\
\hline
\end{tabular}

Table 5.1: Datasheet for the M/A-Com MA4E1317 Schottky diode [44].

The MA4E1317 has low total capacitance $(.045 \mathrm{pF})$ and series resistance $(4 \Omega)$, 
minimizing parasitic losses. The datasheet also cites an operating frequency of up to $80 \mathrm{GHz}$ for this diode, more than sufficient for our purposes. The forward voltage cited to be around .7 V, a typical value for most diodes [44]. A lower forward voltage would be desirable, and in considering future improvements to the system, this is definitely a point to consider.

\subsection{Antenna Design}

A printed circuit board (PCB) half-wave dipole was chosen as the antenna for the system. The copper trace patterned on the FR-4 substrate acts as the antenna. The MA4E1317 is a flip-chip device, and is only suitable for mounting on a PCB. The design and specific parameters of the antenna is based on the dual frequency WPT rectenna in [45], but has been modified for our implementation. The design of the rectenna is shown in Figure 5-1.

The rectenna is composed of three surface-mount components. The MA4E1317 diode acts as the rectification and harmonic generation components. A $47 \mathrm{pF}$ highfrequency ceramic capacitor acts as a low-pass filter, blocking microwave signal from reaching the load. Finally, a $330 \Omega$ resistor is used as a load for experimental purposes. Output pins also attached for measurement purposes. The antenna is designed for operation at $5.45 \mathrm{GHz}$, so the length of the dipole is $27.55 \mathrm{~mm}$, half the free-space wavelength of the incident wave.

The topology of the diode was chosen to maximize rectification efficiency. The Schottky diode is positioned shunt to the feed-in lines of the rectenna. This topology 


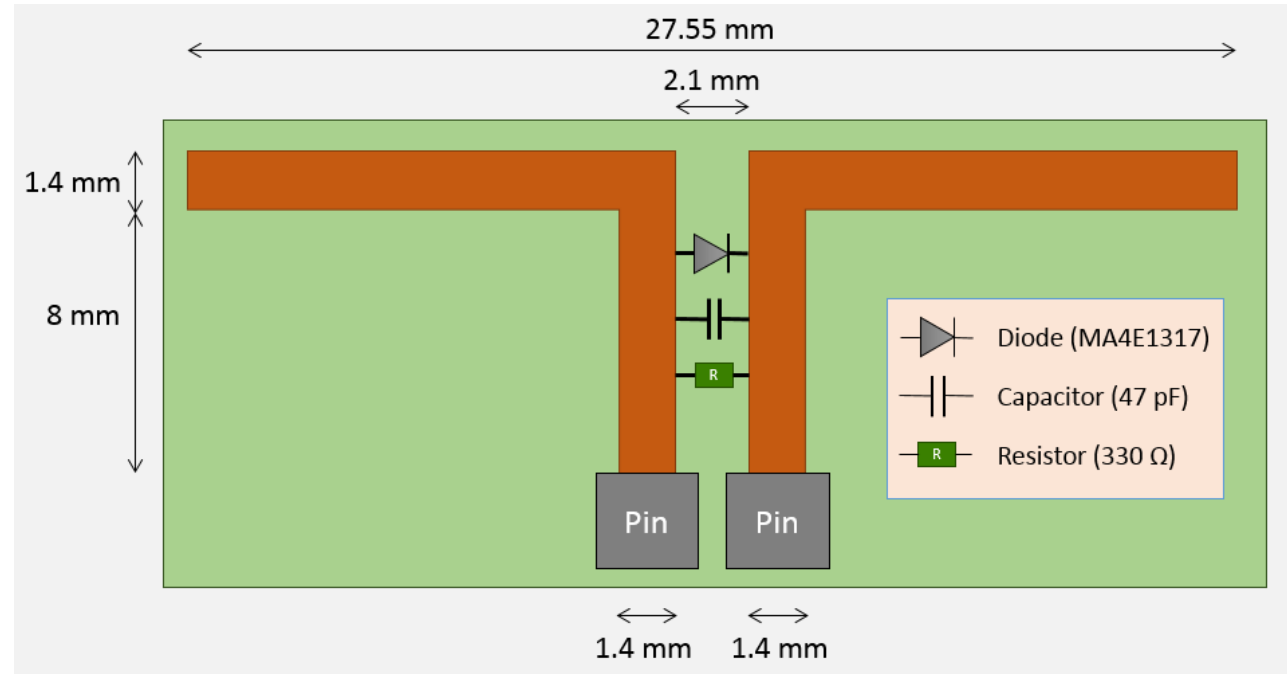

(a) A schematic of the PCB rectenna design and component specifications.

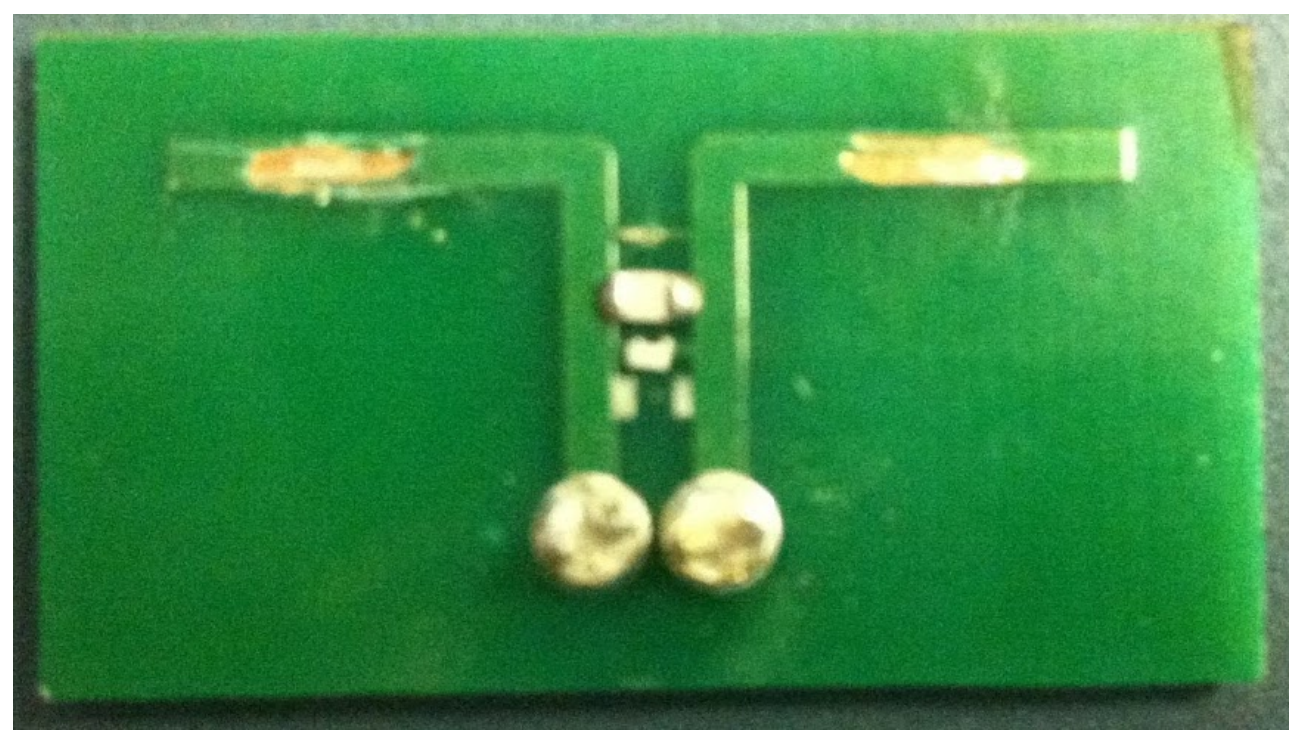

(b) Image of the assembled rectenna

Figure 5-1: Rectenna design 
was chosen instead of a voltage doubler (a two diode configuration) because of its inherently higher power efficiency. However, if higher voltage levels are necessary for a load device, the voltage doubler would be a better choice [46]. Both options should be considered for future iterations of our design.

One important distinction for this rectenna design is the lack of band-pass filtering for higher-order harmonics. This feature is common in most rectennas, but is deliberately left out to allow the harmonic response of the diode to escape through the dipole.

It is worth noting, however, that we did not attempt impedance matching between the antenna and the rectifier components. This is an important point to consider for future work, as proper matching of the components should significantly improve the efficiency of rectification.

\subsection{Rectenna Testing}

To thoroughly test the rectenna, distinct tests were run in order to verify both its rectification and harmonic generation capabilities. We examined a range of frequencies for all tests to isolate peak performance.

\subsubsection{Power Characterization}

To determine the overall rectification efficiency, a second antenna was used to broadcast energy to the rectenna. A $17 \mathrm{dBm} \mathrm{CW}$ signal was generated from the PSG and broadcast through a monopole antenna. The rectenna was then positioned 
parallel to the broadcast antenna, at a distance of $2 \mathrm{~cm}$, and the average DC voltage and power was measured over the load resistor. The test was repeated over a range of broadcast frequencies from 1 to $7 \mathrm{GHz}$. The setup is shown in Figure 5-2a and the results are shown in Figure 5-2b.

Rectification was most pronounced in the $3-5 \mathrm{GHz}$ frequency band. Rectified voltage is highest at $3.4 \mathrm{GHz}$ and $4.6 \mathrm{GHz}$, with $\mathrm{DC}$ voltage levels of $66.5 \mathrm{mV}$ and $59.0 \mathrm{mV}$ respectively. Unfortunately, DC voltage results of higher frequencies were unreliable and erratic given the measurement techniques, and accurate data could not be collected.

Given the resistive load of $330 \Omega$, rectified DC power can be calculated from the voltage above. Using DC voltage $(V)$ and load resistance $(R)$, the average DC power $\left(P_{d c}\right)$ was calculated using Equation 5.1:

$$
P_{d c}=\frac{V^{2}}{R}
$$

A plot of the resulting average DC power delivered to the load as a function of frequency is shown in Figure 5-3.

Considering the input power of $17 \mathrm{dBm}$ (around $50.12 \mathrm{~mW}$ ), the wall-to-load efficiency of the experiment is extremely low for all frequencies. These losses can come from a number of sources, including bad coupling between the broadcast antenna and rectenna, reflection from broadcast antenna, losses in coaxial connections between components, and power radiated away from the rectenna, among others. However, the inherent losses of the experimental setup are not relevant to the overall efficiency of the system; for this, the DC rectification result must be compared to only to power 


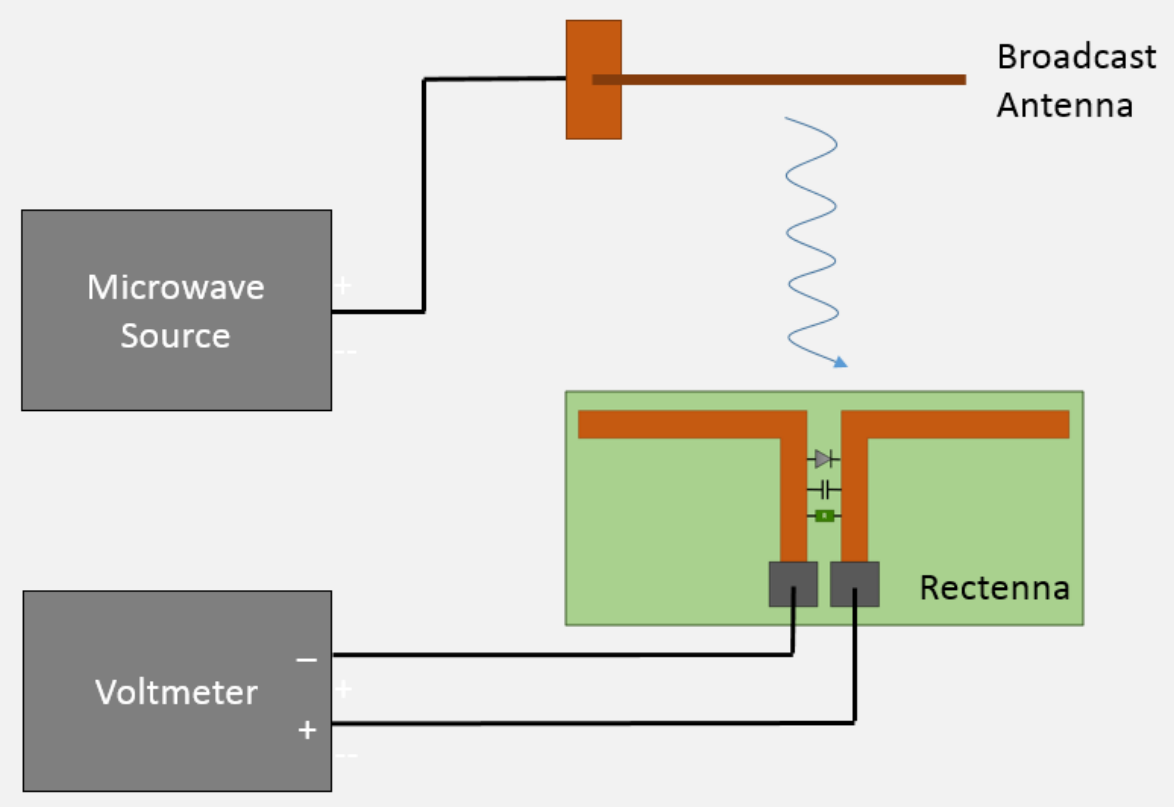

(a) Experimental setup for rectification testing

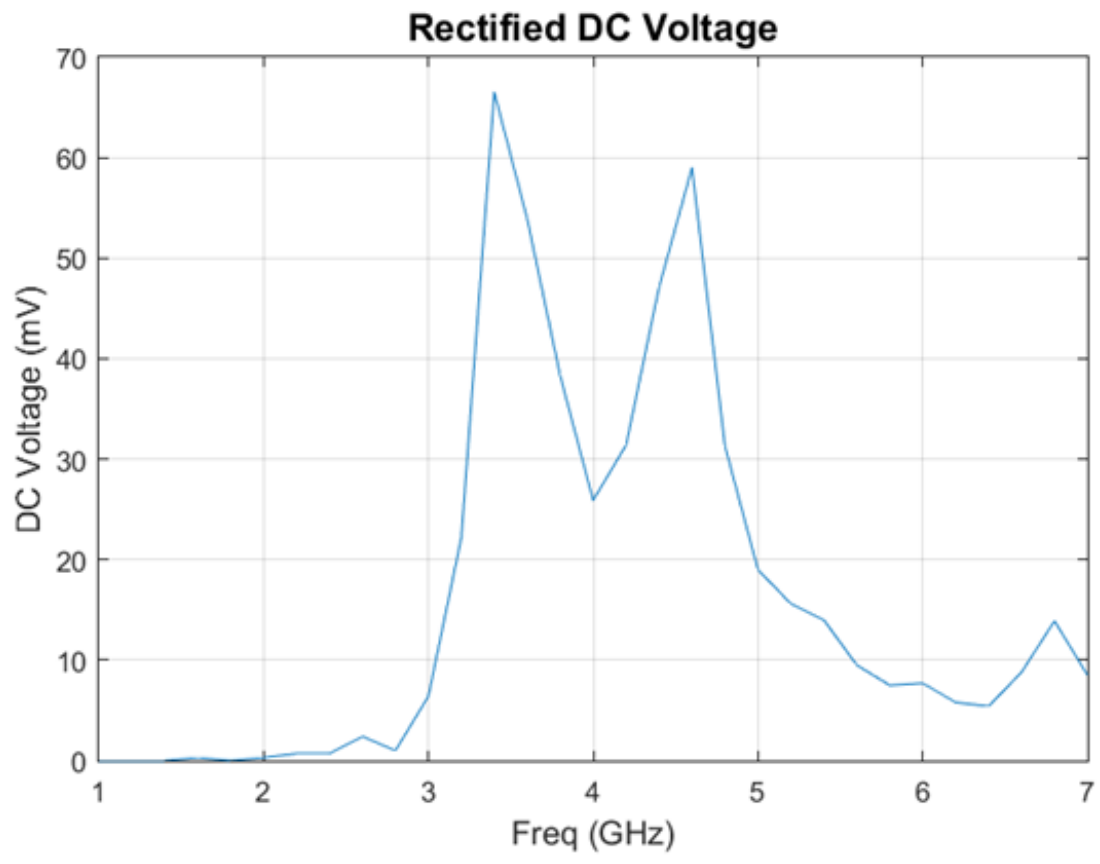

(b) Average DC voltage measured from the voltmeter in the setup on the left for a range (1-7 GHz) of frequency inputs.

Figure 5-2: Rectenna rectification testing 


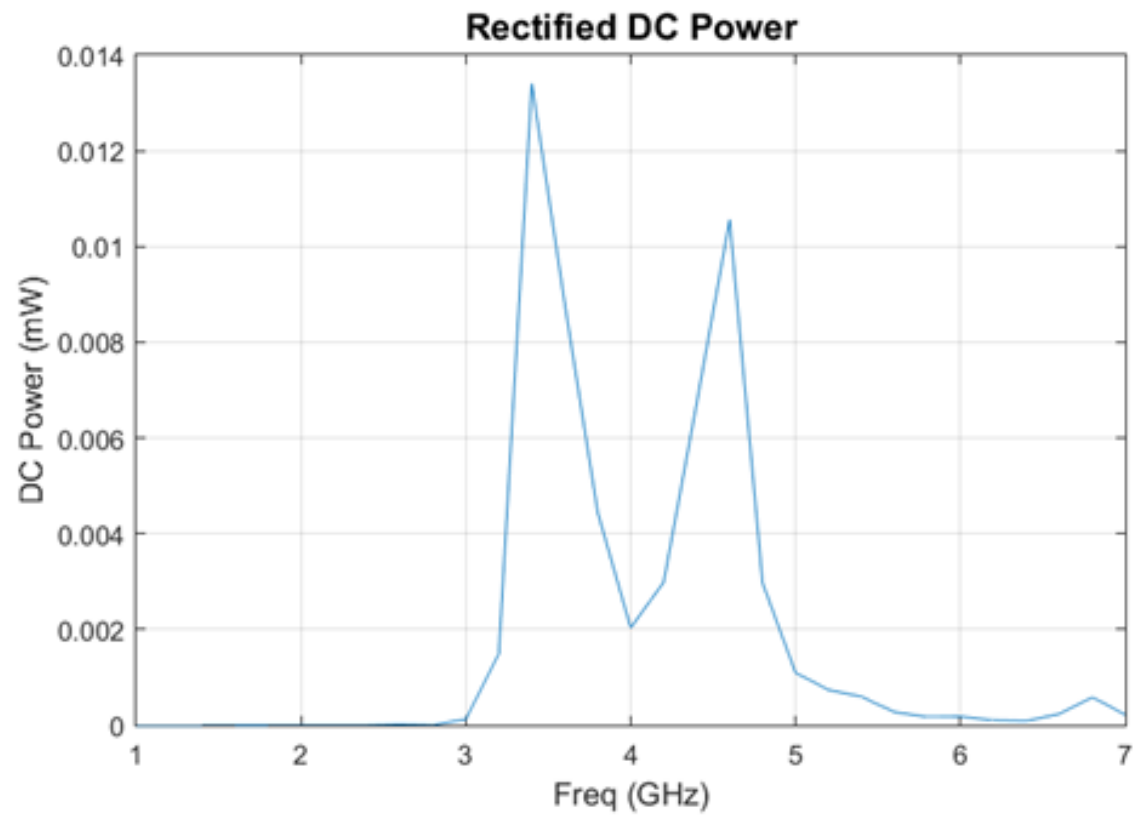

Figure 5-3: Average rectified DC power calculated from DC voltage using the relation: $P=\frac{V^{2}}{R}$

accepted by the antenna.

To establish rectification efficiency, $\mathrm{AC}$ power tests were conducted on a bare dipole antenna with no diode, resistor, or capacitor. The results of this test establish the overall power accepted by the rectenna, representing the peak power available for rectification. These results are directly comparable to the previous DC power results.

The setup of the AC power experiment was similar to the DC power experiment, with the exception of two key differences: the voltmeter was replaced by a DS091304A oscilloscope, and the rectenna was replaced by the bare dipole antenna. In the absence of the $330 \Omega$ load resistor on the rectenna, the $50 \Omega$ input impedance of the oscilloscope [47] was used as the load.

Using $\mathrm{AC}$ voltage amplitude $\left(V_{\max }\right)$ and load resistance $(R)$, the accepted $\mathrm{AC}$ power $\left(P_{a c}\right)$ was calculated using Equation 5.2: 


$$
P_{a c}=\frac{V_{\max }^{2}}{2 R}
$$

The setup and results of the test are shown below in Figures 5-4a and 5-4b, respectively.

Assuming that the transfer function between the antennas doesn't change between the AC and DC tests, these results allow the rectification efficiency to be calculated. The efficiency $(E)$ in this case is the ratio of the rectified DC power $\left(P_{d c}\right)$ to the total accepted $\mathrm{AC}$ power $\left(P_{d c}\right)$, as in Equation 5.3:

$$
E=\frac{P_{d c}}{P_{a c}}
$$

Figure 5-5 shows the efficiency calculated this way, across the range of frequencies tested.

Analysis of the graph shows the same peak rectification efficiencies at $3.2 \mathrm{GHz}$ and $4.6 \mathrm{GHz}$, with $4.7 \%$ and $4.4 \%$ respectively. This rather low efficiency is expected given the lack of optimization and impedance matching in the rectenna. However, this result clearly demonstrates rectification of microwave power to DC power.

\subsubsection{Harmonic Generation}

Harmonic generation was tested by measuring second harmonic power reflected from the rectenna. A microwave source produced a $10 \mathrm{dBm}$ CW signal, which was broadcast from a bare antenna of the same length as the rectenna. The rectenna, positioned around $1 \mathrm{~mm}$ away in the same orientation as the broadcast antenna, should passively generate second harmonics from this fundamental signal. These second harmonic reflections were collected by the bare antenna, passing through a 


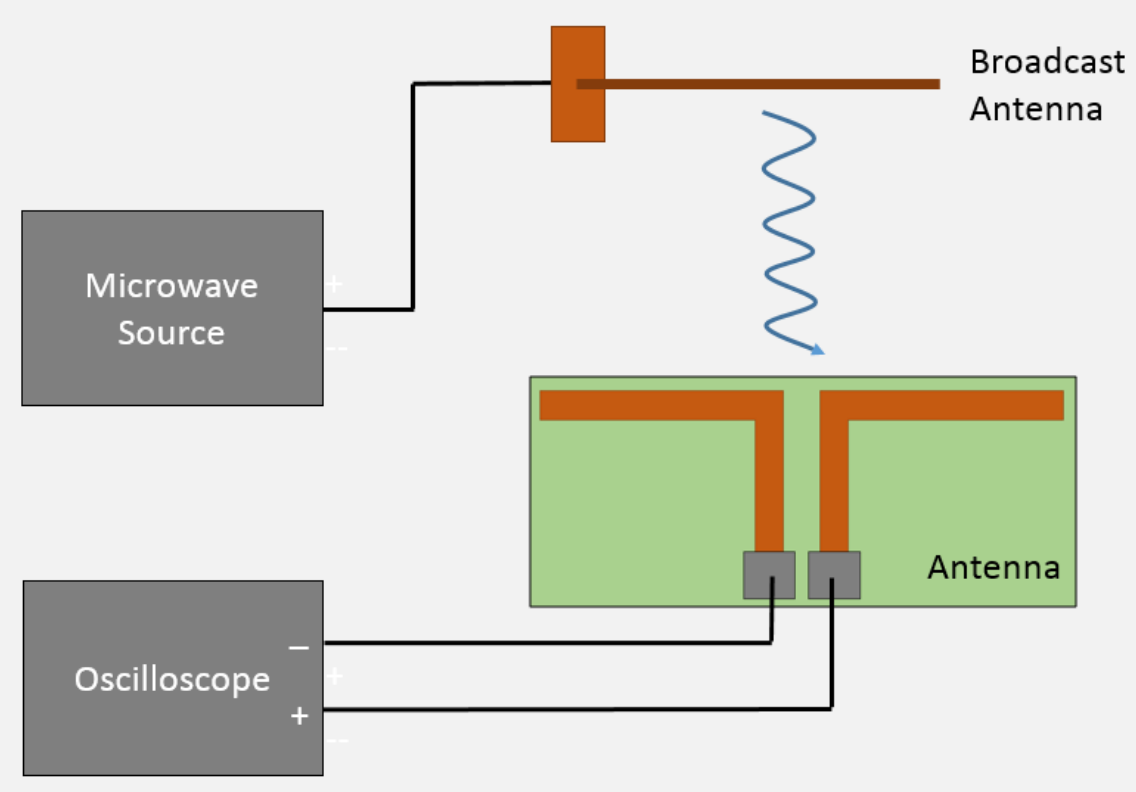

(a) Experimental setup for measuring accepted AC power

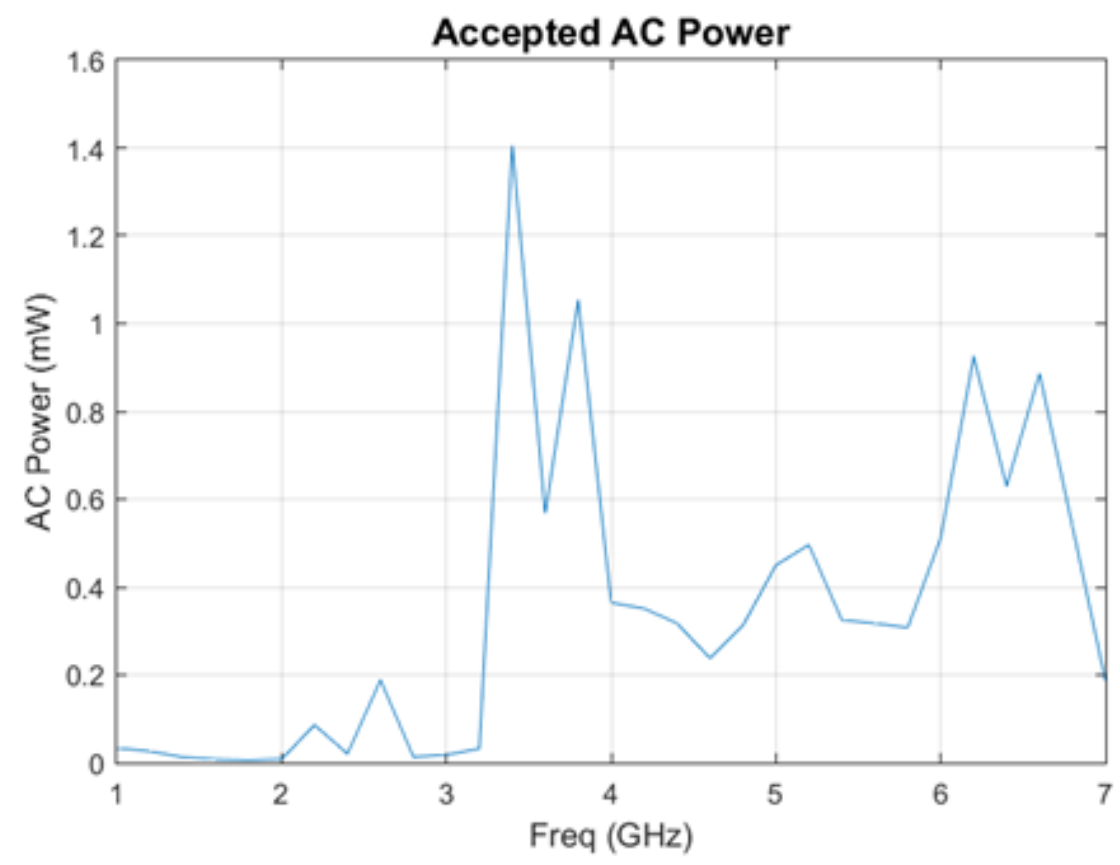

(b) Accepted AC power, used to establish a baseline for efficiency calculation, calculated using the relation $P=\frac{V_{\max }^{2}}{2 R}$.

Figure 5-4: Measuring accepted AC power 


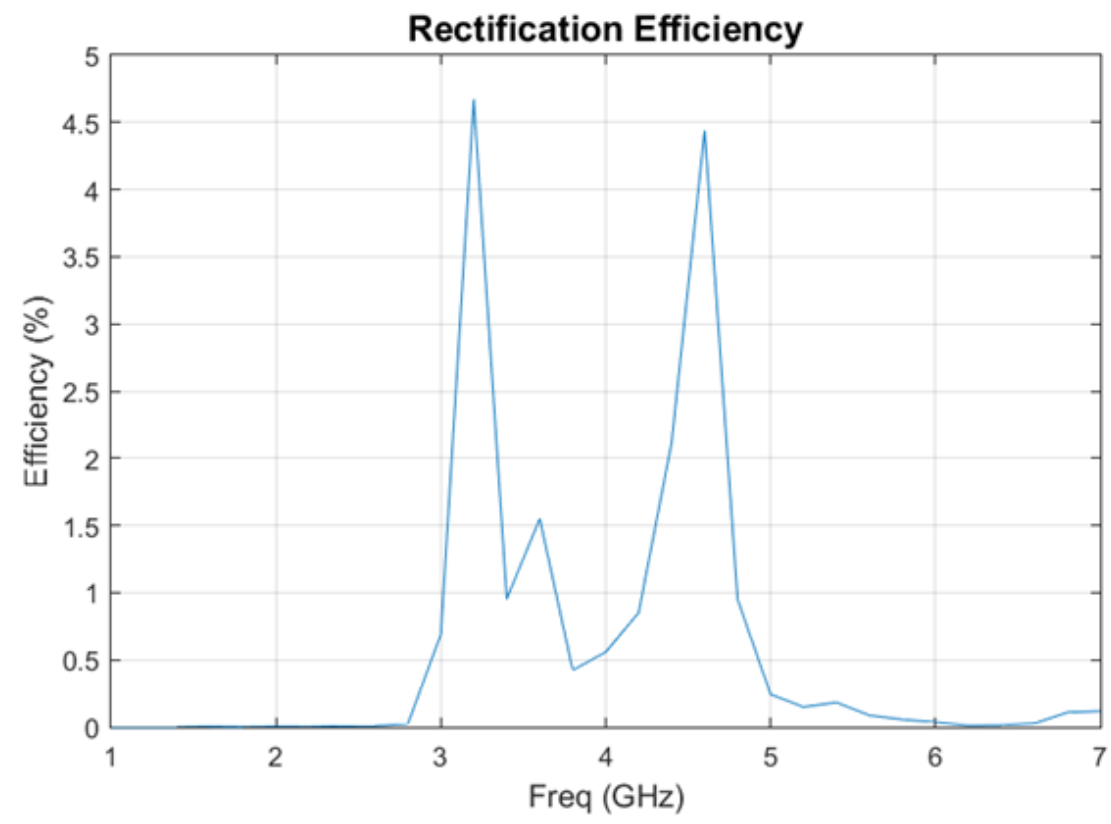

Figure 5-5: Rectification efficiency, calculated using accepted AC power as a baseline. Calculated using the ratio of rectified DC power to accepted AC power.

directional coupler and into a spectrum analyzer for measurement. The test setup and results for this experiment are shown in Figure 5-6.

As seen in the experimental setup, harmonic responses from microwave source were filtered out with a combination of high and low pass filters. A distinct 2nd harmonic was found at $2.45 \mathrm{GHz}$ at $-150 \mathrm{dBm}$, distinctly higher than to the noise

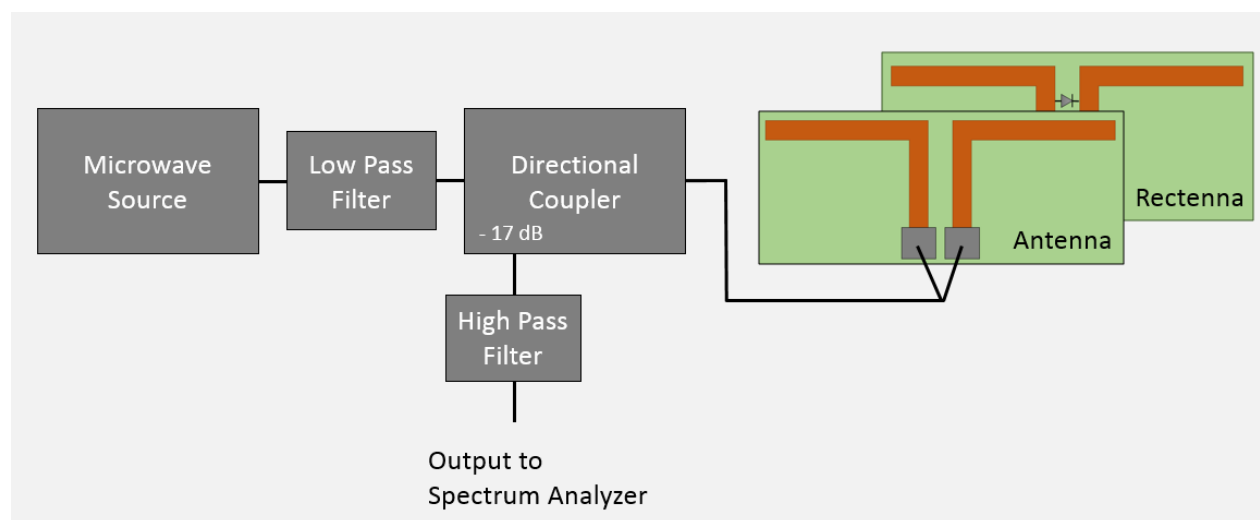

Figure 5-6: Experimental setup for harmonic generation testing. 
level power of $-161 \mathrm{dBm}$. Bandwidth restrictions of filtering components prevented analysis of a full frequency spectrum, and thus, $2.45 \mathrm{GHz}$ is the only frequency we were able to probe for harmonics. Even so, this result does demonstrate the harmonic generation capabilities of the rectenna.

\subsection{Discussion}

The intent of the rectenna was twofold. The rectenna was designed to rectify RF signals (1-10 GHz) and generate higher-order harmonics. Both of these qualities are needed for the creation of an NLTR rectifier. The current experiments establish a baseline for rectification and harmonic generation that can be used for future designs.

The current experiments establish a baseline for rectification and harmonic generation that can be used for future designs. The current design has made no steps toward the impedance matching of the antenna to the components. This is likely a major source of power lost by the system for rectification, and should be a top priority to consider in subsequent designs. While rectenna designs vary based on specific application, it is not uncommon for a rectenna to achieve $70-90+\%$ efficiency $[48,49]$. Clearly, there are many improvements that need to be made to bring our $5 \%$ efficiency to these much higher values. Future rectenna design may benefit from physically splitting the base signal from its harmonic so that the two signals may have dedicated circuitry for each goal. The base signal would be optimized for harmonic generation while the $2 \mathrm{f}$ harmonic input would be optimized for rectification. One such method is using a multiplexer to split incident signal into a $1 \mathrm{f}$ and $2 \mathrm{f}$ signal [ku-band]. This 
system would not suffer parasitic losses due to frequency mismatch between the $1 \mathrm{f}$ and $2 \mathrm{f}$ circuitry.

Our design of a dual-purpose rectenna has satisfied our initial goals, and is a first step towards a functioning NLTR based WPT system. It is able to receive the initial interrogation pulse and generate a 2 nd harmonic response. It is also able to receive the high power pulse and rectify the signal, providing the load device with DC power. Due to the nature of NLTR, the receiving devices do not require power to facilitate either process. After considerable optimization and miniaturization, the rectenna would be used as a general-purpose receiver for the electronics we want to power. 


\section{Chapter 6: Future Work}

\subsection{Vision of a WPT System using TR}

This research represents a first step in the exploration of building a time reversal WPT system. We demonstrate one possible realization of this idea in Figure 6-1.

The proposed system consists of two basic components. The first is a rectenna embedded in a client device. The system as described in Section 3.2 would require an out-of-band feedback channel between the receiver and transmitter. However, we have demonstrated in Section 4.4.3 that a transmitter can target receivers entirely in-band. Our system in Figure 6-1 builds on these findings. The second system component is a charging station that performs the time reversal process. This component is responsible for recording characteristic signals from the receiver(s), time reversing the signals, and re-broadcasting them into the environment.

In a practical system, the rectenna will be integrated into the hardware of a mobile device, or into an external component that plugs into the battery. The charging station would be connected to an external power source, but could otherwise be located anywhere in the room. This system will be most effective in a low-loss scattering environment.

We have demonstrated the underlying concepts of the WPT system depicted in Figure 6-1. However, much work is necessary to transform this proof-of-concept 

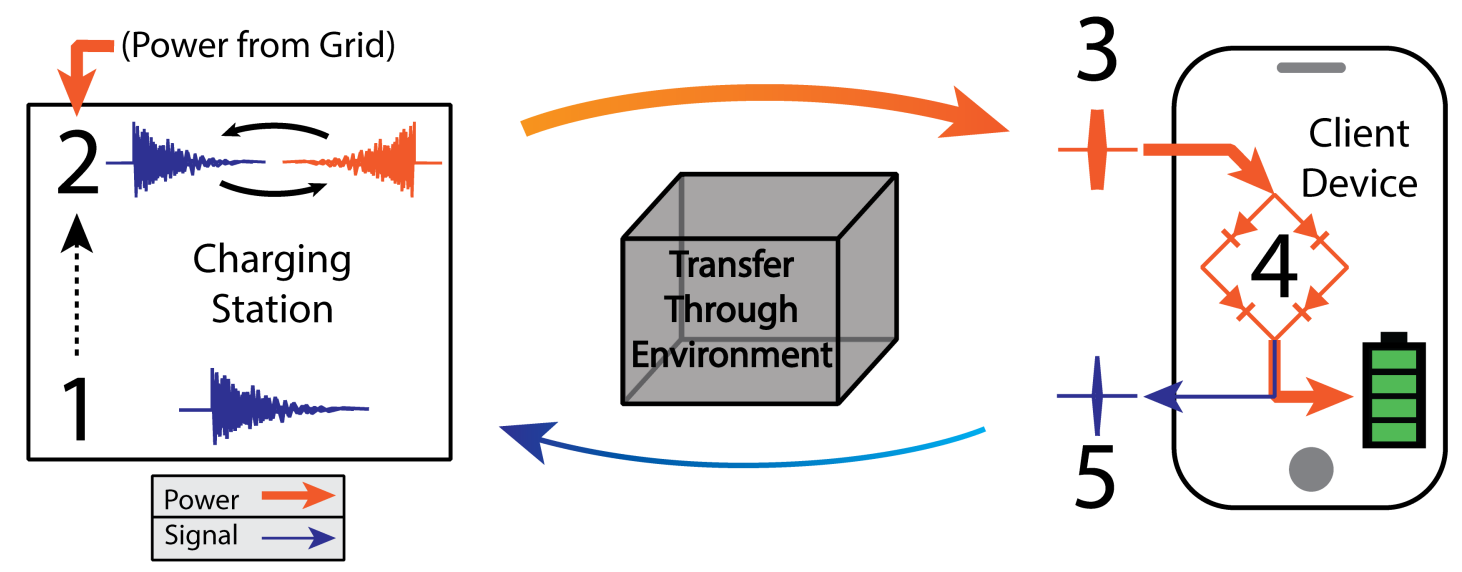

Figure 6-1: Proposed time reversal WPT system. The charging station collects a sona that contains spatial information unique to the receiver's location (1). The sona is time reversed (2), amplified, and broadcast back into the environment. The amplified signal reconstructs on the client device (3) and is converted to usable DC power by the rectifier (4). A small fraction of the signal is used to re-broadcast a new characteristic signal (5) into the environment, which will be collected in the next sona, creating a stable loop (1). This loop can be initiatied by a pulse from the charging station, and requires no input energy from the client device.

technology to a functional product that consumers may use. Future work discussed here falls into one of two categories. The first category includes established TR techniques that may be extended into the field of TR WPT. The second category includes novel designs proposed by the team applicable only to TR WPT.

\subsection{Applying other techniques from TR to WPT}

Imaging of objects and non-invasive surgery are already common applications of TR. As a result, many techniques have been developed to improve reconstruction quality on a target. We suspect that many of these techniques may have applicability to our proposed system, but did not have the opportunity to investigate them in our research. However, TR methods are generally designed for targeting stationary 
objects, in unchanging environments. These methods may have novel advantages and disadvantages for TR WPT applications. We suggest that future research be dedicated to investigating the applicability of techniques such as iterative time reversal and exponential amplification to a practical WPT scheme.

\subsubsection{Iterations}

It has been well proven in the literature that time reversal focusing on an object can be repeated to improve waveform collapse on a target. [23] However, this technique has not yet been applied to TR WPT.

We suspect that iterative TR can improve the reconstruction of a signal over time. The side lobes in the time domain are representative of energy that may not be able to be rectified unless they are combined into the main peak. We predict that we can maximize our efficiency and reconstruction quality using this method. Additionally, we predict that this method will only be useful for stationary targets. Because iterating the time reversal process takes several more steps, the length of time required to focus on a target is increased considerably. Since the technique for focusing upon a moving receiver proposed earlier relies upon repeating the time reversal process many times, it may not be acceptable for the time required to perform TR to increase several hundred percent.

Both of these characteristics can be easily tested, using algorithms already applied to acoustic TR. An experimental setup very similar to ours could be applied to this research. A faster sona refresh rate would be required from the TR system for this method to be able to outpace the natural "decay" of the testing environment. Once 
an iterative algorithm has been tested and found successful it should be tested in a less homogeneously reflective environment, to determine it's performance in lossy environments.

The novelty of such research is primarily in the characteristics measured. The demonstration (or lack thereof) of filtering of lossy paths would also be a major finding. Models should be generated relating the sona refresh rate to performance gains using iterative method. If possible, multiple environments should be tested to generalize these results.

\subsubsection{Multiple Transmitters}

All of the work in this thesis was conducted using a single transmitting antenna. The incorporation of multiple antennas would haved required major modifications to our experimental setup, and thus was beyond the scope of our study. However, previous work in time reversal in other fields, such as acoustics, have demonstrated the ability to improve signal quality through the use of multiple transmitters.

In one study, Mathias Fink demonstrated that a single-channel time reversal mirror acts like a spatiotemporal matched filter, where the time reveresal process is a convolution of the injected signal with a time reversed "image" of that signal. Using a 96-element antenna array, Fink showed that using multiple channels created a stronger collapse at the reconstruction time and that it tended to cancel the contributions of the temporal side-lobes, ultimately providing a better peak-to-noise ratio. When the number of channels is sufficient, the result is an inverse filter, which should produce the ideal reconstruction [50]. 
Although a practical wireless power transfer system will likely not be able to reach this theoretical limit, the positive relationship between number of elements and reconstruction strength should hold: namely, that adding $N$ elements to the time reversal mirror should increase the reconstruction strength by at least a factor of $N$. In addition, it should reduce the accidental creation of "hot spots" at locations other than the intended reconstruction point, which would be a necessary safety concern to address in a practical system.

\subsubsection{Exponential Amplification}

Exponential amplification can be used to counteract decreased reconstruction quality caused by system losses [51]. Exponential sona amplification is a nonlinear amplification to the sona across its timespan. It is meant to counteract the larger losses (due to larger number of reflections) experienced by reflective orbits with larger paths.

However, it should be understood that this method only compensates for the losses experienced by a time reversed signal. It does not avoid losses altogether, and in fact more energy is lost through this method. As a result, this method sacrifices efficiency for reconstruction quality. This tradeoff could be significant to designers of a TR WPT system. It would be useful to quantify the effects of this tradeoff in a WPT context so that informed decisions can be made in the design of future systems. 


\subsection{Novel Advancements for TR in a WPT context}

There are aspects of a TR WPT system that create avenues for research and innovation. Many of these take advantage of the unique operating principles with a TR WPT system. Most TR applications care little about the environment, focusing only on the direct link between transmitter and receiver. In TR WPT the opposite is true. Most TR applications consider stationary - or near stationary - targets. TR WPT system must always consider moving targets, or at least stationary targets within a dynamic environment.

These and other considerations set TR WPT apart from other TR research, and require the development of novel techniques. Below we suggest several research topics specific to TR WPT, experiments that could explore them, and how the results of these experiments can benefit TR WPT.

\subsubsection{Sub Cavity}

It is clear to the team that a TR WPT system will likely need some environmental modifications to achieve an efficiency sufficient to see practical application. These modifications should also be designed to be as cheap and simple to install as possible, to ensure that system price remains practical.

We suggest a method of improving efficiency of TR wireless power transfer by introducing a "sub cavity" within the transfer environment. This "sub cavity" will be defined as a region of the environment that allows extremely efficient TR WPT. The sub cavity becomes much less lossy than the environment as a whole. TR paths 
will preferentially move through the sub cavity, and can dramatically improve the efficiency of the entire system. A successful sub cavity must have the following characteristics:

- High transfer efficiency/low reflective losses

- Chaotic geometry to facilitate the function of TR

- Presence of one or more paths between the sub cavity and main environment

These qualities already exist in many environments. Many indoor environments contain metallic objects, either as part of the environment itself, or embedded within the walls of an environment. Most of these objects tend to be within a meter of ground level. Thus, the lowest meter of a room could be exploited as a sub-cavity in practical applications. A study conducted at USC characterizizng RF propagation through common building materials at a range of frequencies confirms that many of these common materials provide high reflectivity in practice (see Tables 2 and 3 in particular) [52].

Experiments should be conducted to quantify the benefit of these materials in the context of a TR WPT system. First, efficiency should be measured in completely empty rooms composed of typical materials. Then individual items hypothesized to affect (both positively and negatively) TR WPT efficiency should be introduced individually. The position and arrangement of these items should be altered, and their impact on efficiency quantified. Finally, the position and arrangement of groups of items should be altered as well.

We hypothesize: 
- The position of an individual object becomes a negligible influence when it is more than 0.5 meters from a charging station or client

- Quantity of reflective objects is more important than their size (surface area)

- Large numbers of reflective objects decreases the impact of loss elsewhere in the environment

If this experiment demonstrates an unacceptably low transfer efficiency in an ordinary environment, the creation of an artificial sub cavity may be necessary.

A proposal for an artificial sub cavity can be found in Figure 6-2. This cavity is a thin sheet of material that would be mounted to the ceiling of the room in question. The material in this sheet should have a smaller index of refraction than air; as a result any signal broadcast into can become "trapped" in the layer. At any given point along the barrier of the sub cavity only small amounts of signal will return to the main environment. A TR WPT system can selectively choose paths through the sub cavity that will focus on a given receiver with high transfer efficiency. Reflectors can be added to the sub cavity as needed to ensure that its complexity is enough to allow TR to occur.

Research would focus on, firstly, proving that sub cavities can work for the purposes of increasing efficiency of transfer. Once this has been established, the geometry of the sub cavity should be optimized to work in a wide range of potential environments. Uniformity of signal coverage is a major concern, and must be tested either experimentally, or through use of simulation software. 


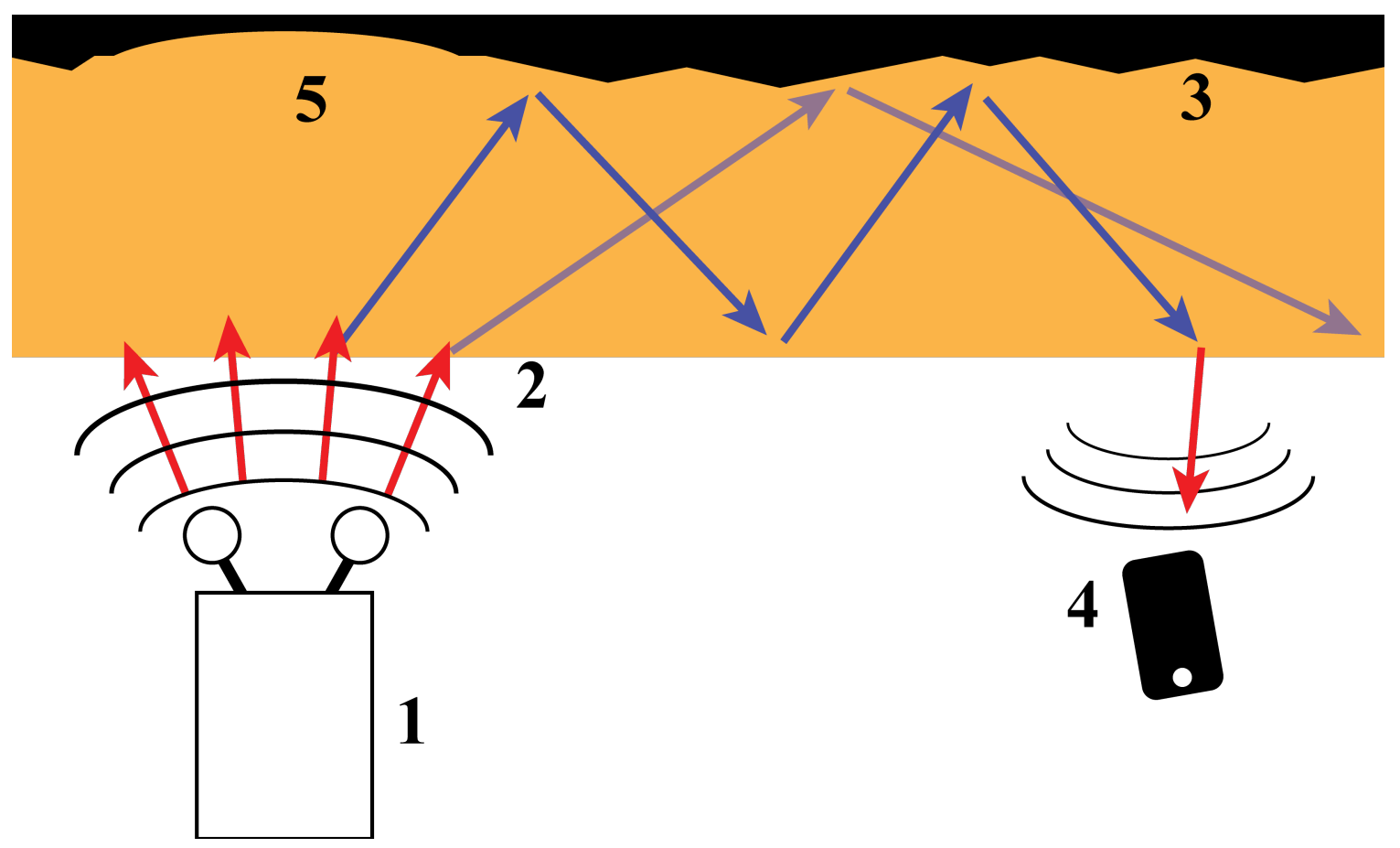

Figure 6-2: A proposed sub cavity design. A transmitter (1) broadcasts an interrogation pulse into the environment. When the pulse reaches the low index of refraction material of the sub cavity, it refracts, taking on a lower angle (2). The interrogation pulse totally internally reflects within the cavity (3), until returning to the main environment. Some aspects of the signal reach the receiver, allowing a TR link to be established (4). A focal aspect of the geometry above the transmitter (5) is highlighted as a way of improving transmission.

\subsubsection{Protection of Information}

Many of the improvements suggested above are focused on increasing the efficiency of the final system. Most of these improvements assume that while the system may be lossy, it is enclosed. That is, while signals may be absorbed by the environment, they are not lost completely. However, complete loss can be expected to occur often in practical applications. This can be due either due to holes in the environment (such as windows or doors) or heavily absorptive materials. The use of a selective transmission channel (as proposed above) may mitigate the effects of a channel through which a 
signal may escape. However, other methods should also be considered.

Earlier our concern with losses focused primarily on their impact on efficiency. Information transfer was sufficient to allow TR to occur. However, complete losses remove information from the system, and severely damage the ability for TR to reconstruct on a target. This can be visualized using the idea of loss of outgoing paths. Figure 6-3 shows how time reversal can be impacted by environments with complete loss.

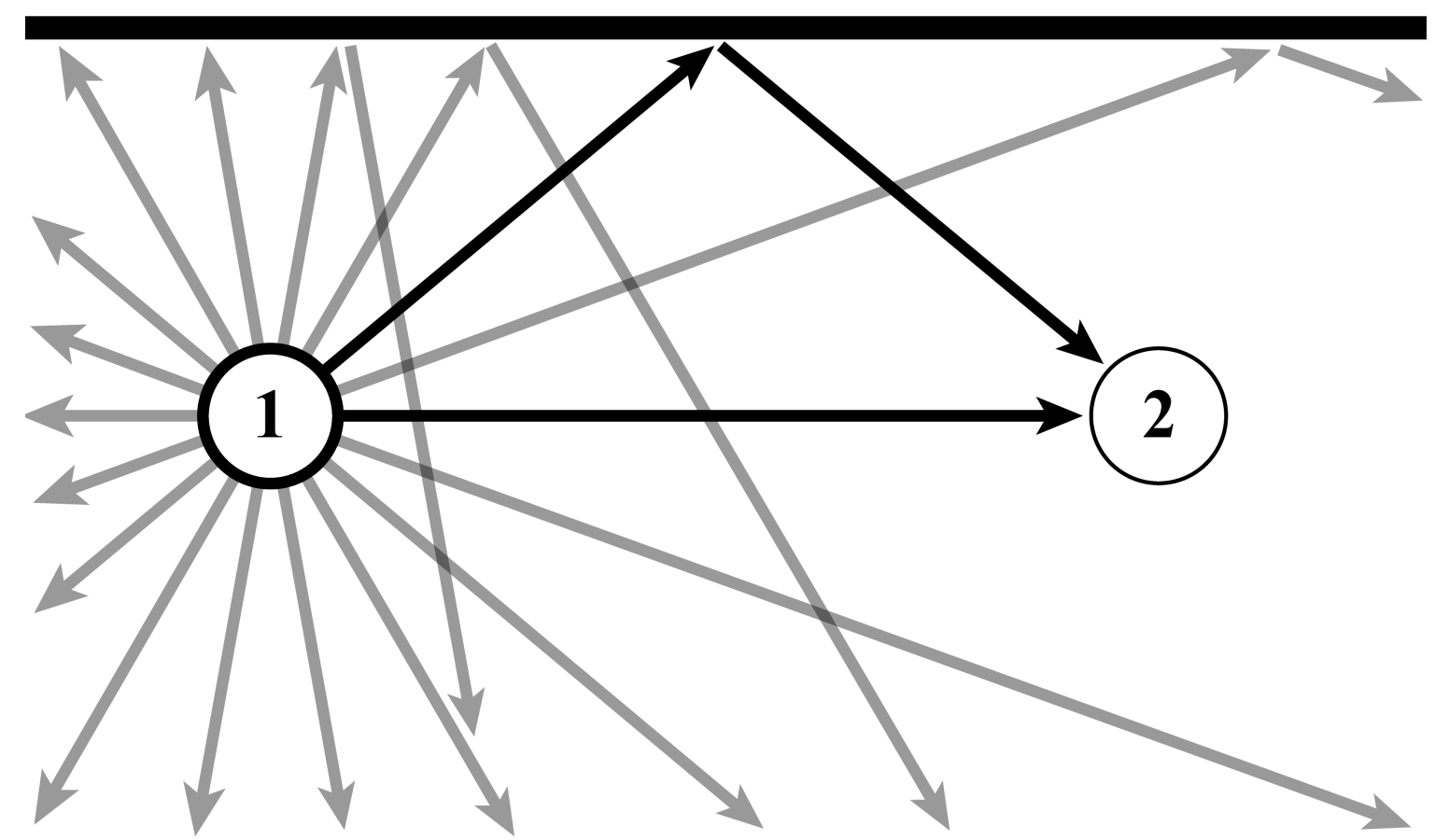

Figure 6-3: A representation of how complete losses affect time reversal. In this scenario, a transmitter (1) sends a signal in all directions in an environment with only one reflective surface. As can be seen, only a small percentage of the energy successfully reaches the receiver (2). If time reversal from (2) to (1) were to be attempted, a small and spatially unbalanced amount of energy would arrive at (1), resulting in a poor reconstruction. 
This is partly why a large number of reflective surfaces is beneficial in a time reversal environment. However, we believe that this effect is more advantageous (from an information protection point of view) when it occurs near to the target of the time reversal process. This concept is discussed in Figure 6-4. Note that there is an issue with how much of tine incoming signal may be reflected on approach - we do not currently have a solution to this problem. Using an array of transmitters to increase the number of possible short orbit paths to the receiver should also mitigate the effects of information loss.

We believe that this concept should be tested using simulation methods as a proofof-concept. Should it function as expected, we propose moving forward with some sort of experimental demonstration of information preservation effects. Finally, a model should be created describing the effectiveness of the method. Particular attention should be paid to the size of the design - we expect this method to be difficult to miniturize.

\subsubsection{TR WPT applied to Internet of Things Devices}

Previously we have primarily considered how TR WPT could be applied to mobile devices. Mobile devices are ideal for TR WPT because they are generally used in enclosed areas, use relatively low power, and benefit from the removal of wires. However, there are other applications that may also benefit from the system proposed.

In particular we would like to consider how TR WPT could be applied to to internet of things devices. Internet of things depends on a large number of interlinked computers managing consumer and industrial machines. Internet of things devices 


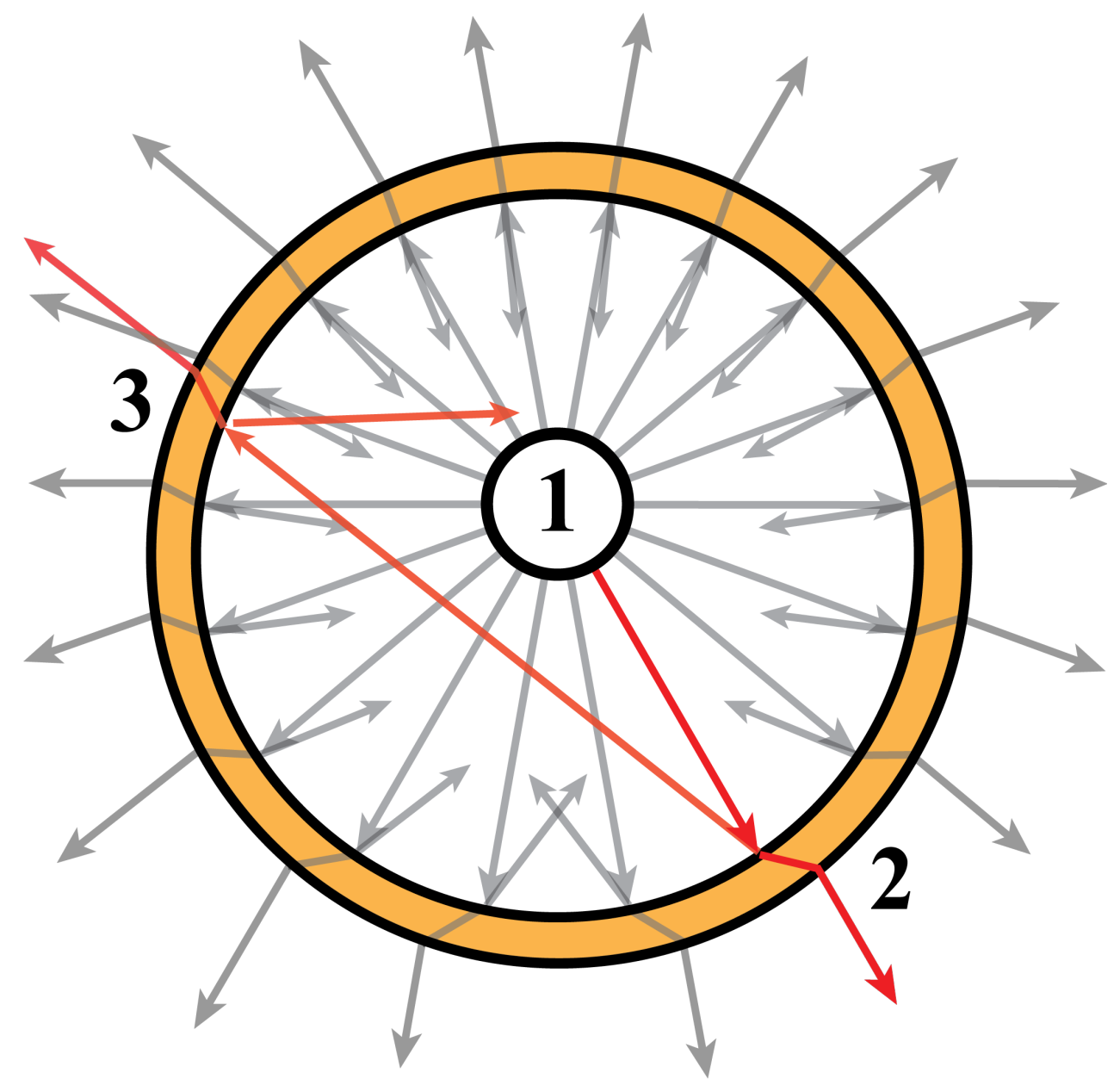

Figure 6-4: A proposed method of protecting a TR system from loss of information. The "target" of the TR process (1) is surrounded by a surface of semi-reflective material. This surface reflects effectively "splits" the outgoing wave into several smaller pieces (2). The result is that a greater solid angle is preserved coming out of the antenna/reflector system in cases such as the one in Figure 6-3.

are useful in automating simple tasks, and for collecting environmental data. These devices are dependent on continual connection to the internet; as a result their power demands tend to require them to be tethered to wall sockets, or often-replaced batteries. However, their power demand is low, and could be addressed using TR WPT even easier than mobile devices.

We propose that these devices could serve as the inspriation for an experiment into 
practical TR WPT. In particular, these devices give an opportunity to experiment how selective targeting can be used with groups of devices. In Section 4.4.3 we demonstrate how selective targeting can be done. However, applying this technique to groups of devices will require a more sophisticated technique than demonstrated here, and will serve as a novel extension of this research. Such an experiment will be an important first step in quantifying the efficiency of a TR WPT system in a practical scenario. 


\section{Chapter 7: Conclusion}

\subsection{Contributions}

The various elements of this thesis serve to display the separate aspects of a wireless power system that can be accomplished using time reversal. We successfully demonstrated six significant aspects to time reversal throughout our thesis:

1. Mapped the spatial profile of a reconstruction

2. Performed time reversal on a moving target

3. Decreased the time between reconstructions and increased the power delivered by overlaying sona signals

4. Proved that NLTR may be generalized to an arbitrary number of nonlinear objects

5. Developed a method for selective targeting using NLTR

6. Set forth a design for a dual purpose rectenna

Mapping the spatial profile of a reconstruction is perhaps our most significant contribution. As is the case with any WPT technology, safety is a principal concern. By understanding the spatial profile of a reconstruction, we are able to accurately 
address the effects of microwave radiation on individuals and electronics nearby a charging device. We believe that this profile is generalizable from LTR to NLTR, making the spatial profile a powerful tool for future work in both NLTR and rectenna design.

The proof-of-concept work in overlapping sonas demonstrates that a WPT technology based on time reversal would require rapid pulsing of power instead of a continuous amount of power being transferred. This has important implications for the integration of rectennas into a TR WPT system. Having a simple method for controlling the time between reconstructions provides an avenue for optimizing a future rectenna system. This also provides another tunable variable to optimize a WPT system.

We showed in Chapter 4 that the strength of the nonlinear reconstruction is dependent upon the strength of the nonlinear sona generated from the nonlinear object. Thus, a more distinct harmonic response in the rectenna is very desirable for both selective targeting as well as simultaneous reconstruction. Careful design of the dualpurpose rectenna will be needed for a successful NLTR WPT system.

\subsection{Evaluating Time Reversal as a Wireless Power Transfer Method}

Throughout this thesis, we have demonstrated many aspects of TR that may be incorporated into a future WPT technology. Our work focused on proof-of-concept experiments that demonstrate basic functionality and feasibility of the proposed tech- 
nology. We first identified key characteristics of current WPT methods on the market or soon to enter the market. These characteristics were used to identify the following properties to be ideal for a WPT system and have demonstrated the first three throughout our thesis:

1. Ability to function while the receiving device is moving

2. Ability to charge multiple devices

3. Ability to choose which devices receive power

4. Ability to deliver up to 1 watt of power to a device

5. Ability to function without line of sight

6. Maximum range of up to 30 feet

The ability to function while moving was shown in Chapter 3 by utilizing the spatial profile of a time reversed reconstruction. Because the reconstruction voltage changed predictably in space, we were able to deliver a relatively uniform amount of voltage to the receiver antenna as the antenna moved at a constant velocity. By re-collecting the sona signal after a set amount of time, we successfully used time reversal to send power to a moving target. As discussed in Chapter 2, uBeam is the only company that boasts this feature, providing a clear feature that makes time reversal unique in the realm of WPT technology.

In Chapter 4 we illustrate charging multiple devices using NLTR. While every current WPT technology can charge multiple devices, most are dependent upon feedback 
networks, complex algorithms, and/or constant communication between transmitter and receiver. As we demonstrated, NLTR can target on an arbitrary number of nonlinear elements in the time reversed step. Due to this aspect of NLTR, a time reversal based WPT system could charge 10 devices easily without any change to our algorithm. The generalizability of time reversal to an arbitrary number of receivers clearly illustrates both the simplicity and strength needed to be effective in the market for WPT.

We further showed in Chapter 4 that using NLTR, we may select targets to receive power at the exclusion of others. By characterizing the nonlinear response of our model diode, we provided a basic method to produce a reconstruction on a specific diode. We were able to effectively discriminate where the reconstruction oc-

curred under the condition that we wanted to selectively target either a $V_{k}^{\text {high }}$ or $V_{k}^{\text {low }}$ diode. Our experiments in selective targeting demonstrated one possible method of discriminating between receivers using NLTR. We believe that more efficient and more generalizable algorithms may exist for other types of nonlinear devices.

\subsection{Final Words}

By demonstrating the various elements of our time-reversal-based WPT system, and discussing the interrelationship between those elements, we have demonstrated the proof-of-concept for such a technology.

This thesis has demonstrated preliminary evidence that time reversal may be applied to a WPT technology. Our results illustrate that there is still much to be 
discovered in the field of time reversal. The field of WPT is constantly growing, with innovation around every corner. We have discussed one such innovation that may one day revolutionize how we view power, charging, and batteries forever. 


\section{Chapter A: Spatial Profile Fit Parameters}

\begin{tabular}{|c|c|c|c|c|c|}
\hline Frequency & Fit Parameter & $\mathrm{a}$ & b & $\mathrm{c}$ & d \\
\hline \multirow{3}{*}{$2 \mathrm{GHz}$} & Value & 0.05 & 36.27 & -77.20 & 0.10 \\
\hline & Lower Bound & 0.05 & 35.95 & -77.29 & 0.10 \\
\hline & Upper Bound & 0.05 & 36.58 & -77.10 & 0.10 \\
\hline \multirow{3}{*}{$3 \mathrm{GHz}$} & Value & 0.69 & 37.16 & -82.90 & 0.18 \\
\hline & Lower Bound & 0.68 & 37.01 & -83.01 & 0.18 \\
\hline & Upper Bound & 0.69 & 37.31 & -82.79 & 0.18 \\
\hline \multirow{3}{*}{$4 \mathrm{GHz}$} & Value & 2.41 & 30.41 & -75.11 & 0.44 \\
\hline & Lower Bound & 2.38 & 30.33 & -75.18 & 0.43 \\
\hline & Upper Bound & 2.43 & 30.50 & -75.04 & 0.44 \\
\hline \multirow{3}{*}{$5 \mathrm{GHz}$} & Value & 2.18 & 26.72 & -73.58 & 0.43 \\
\hline & Lower Bound & 2.13 & 26.59 & -73.70 & 0.41 \\
\hline & Upper Bound & 2.23 & 26.86 & -73.47 & 0.44 \\
\hline \multirow{3}{*}{$6 \mathrm{GHz}$} & Value & 1.41 & 20.62 & -74.97 & 0.32 \\
\hline & Lower Bound & 1.36 & 20.45 & -75.12 & 0.31 \\
\hline & Upper Bound & 1.46 & 20.79 & -74.83 & 0.33 \\
\hline \multirow{3}{*}{$7 \mathrm{GHz}$} & Value & 0.81 & 16.00 & -74.84 & 0.20 \\
\hline & Lower Bound & 0.78 & 15.85 & -74.99 & 0.20 \\
\hline & Upper Bound & 0.85 & 16.14 & -74.69 & 0.21 \\
\hline \multirow{3}{*}{$8 \mathrm{GHz}$} & Value & 0.47 & 15.07 & -74.90 & 0.11 \\
\hline & Lower Bound & 0.45 & 14.97 & -75.01 & 0.11 \\
\hline & Upper Bound & 0.48 & 15.17 & -74.79 & 0.12 \\
\hline \multirow{3}{*}{$9 \mathrm{GHz}$} & Value & 0.23 & 13.64 & -74.60 & 0.06 \\
\hline & Lower Bound & 0.22 & 13.56 & -74.68 & 0.06 \\
\hline & Upper Bound & 0.24 & 13.72 & -74.51 & 0.06 \\
\hline
\end{tabular}

Table A.1: The table above displays the fit parameters for the spatial profiles described in section 3.4.2. The upper and lower bounds referenced in the table are the bounds for the confidence intervals for the fir parameters to $2 \sigma$.

$$
V(x)=a \cdot\left|\operatorname{sinc}\left(\frac{x+c}{b}\right)\right|+d
$$




\section{Glossary}

active transmission A WPT system where both transmitter and receiver expend some power to maintain the process.

anisotropic scatterer A scattering element with a directional bias.

AWG Arbitrary Waveform Generator.

carrier frequency The frequency of the carrier wave for our interrogation process..

CNAM Center for Nanophysics and Advanced Materials.

collapse The movement of a time reversed sona towards its point of convergence. The collapse occurs as the reflections of the sona interfere constructively at the point of convergence.

CST Computer Simulation Technology: Microwave Studio.

DORT Decomposition of the Time Reversal Operator.

DSO Digital Storage Oscilloscope.

efficiency The ratio of usable energy delivered to the total energy broadcast by a WPT system. How efficiency is measured can vary wildly based on methodology. 
In this paper we will define efficiency as being the total power delivered to a load, compared to the total power drawn by the transmitter.

EM Electromagnetic.

FDTD Finite Difference Time Domain.

FFT Fast Fourier Transform.

FIT Finite Integration Technique.

Gigabox The microwave cavity used for our experimental TRM.

GPIB General Purpose Interface Bus.

HRMC Highly Resonant Magnetic Coupling.

impedance matching Adapting the impedance of a load to the impedance of a power source, such that power delivered to the load is maximized.

injecting port A port designated for broadcasting (injecting) signals. The transmitter of the TRM.

interrogation pulse The signal injected into the cavity at the transmitter in order to obtain a sona.

knee voltage The voltage nescessary for "turning on" a diode.

linear time reversal Time reversal involving a transmitter and receiver. Requires some separate method of communication of sonas between transmitter and receiver. 
LTR Linear Time Reversal.

maximum power The maximum amount of power that can be transmitted to a target using a given wireless power transfer system.

mode An eigenfunction of the wave equation given the boundary conditions of a system.

mode density A discription of the seperation of modes in terms of frequency (energy) separation; as $\delta f=0$ the mode spectrum tends to form a continuum.

NLTR Nonlinear Time Reversal.

nonlinear time reversal Time reversal involving a transmitter and nonlinear receiver. During use the nonlinear receiver will generate nonlinear harmonics allowing the sona that it generates to be filtered out from background noise.

parasitic capacitance The unwanted capacitance introduced from the arrangement of components in an electrical network along with materials.

passive transmission A WPT system where only the transmitter expends power to maintain the process.

port An antenna mounted on the Gigabox, used to transmit signals into the Gigabox, and record signals within it.

PSG Pulse Signal Generator. 
pulse inversion The signal processing technique for isolating the nonlinear component of a signal.

PWM Pulse Width Modulation.

range The maximum distance that a wireless power transfer system can transmit a given amount of power.

ray chaotic environment An important feature of a chaotic system where there is a sensitive dependence on initial conditions; if one were to consider two ray originating at a given point with an arbitrarily small angle in separation, the forward ray traces quickly diverge. For our application, the implication is these ray traces will cover the volume of the cavity.

receiver The component of a WPT system that has a net input of power into the system.

reconstruction A focused signal produced as the result of a TR process. Reconstuctions are formed from the injection of time-reversed sona at the transmitter.

recording port A port designated for recording broadcasts injected into the cavity. The receiver of the TRM.

rectenna A rectifying antenna. It collected electromagnetic power and converts it into usable DC power. This device is commonly used in wireless power transfer systems.

RF Radio Frequency. 
S parameters The parameters of a scattering matrix which describe a linear electronic networks's transmission/reflection of an approaching EM wave.

scatterer An element that functions to isotropically scatter ambient waves. A reflector.

short-orbit The mechanism responsible for the multiple reconstruction signals appearing at different time intervals.

SNR Signal-to-Noise Ratio.

sona Sona: originating from sonabilis, resonant. The signal recovered at the receiver during the interrogation stage of a TR process. During a TR process the sona is time reversed and injected into the cavity to form a reconstruction at a receiver.

time reversal Time reversal can refer to (1) the property of time reversal symmetry in the system; or (2) the signal focusing technique using said property.

TR Time Reversal.

transmitter The component of a WPT system that outputs power into the system.

TRM Time Reversal Mirror.

TRR Reverse Recovery Time.

WPT Wireless Power Transfer. 


\section{Bibliography}

[1] N. Tesla, "On light and other high frequency phenomena," Journal of the Franklin Institute, vol. 136, no. 4, pp. 259-279, 1893.

[2] S. Ogiso, "2013 Toyot Hybrid World Tour - Satoshi Ogiso," Aug. 2013.

[3] "Volvo Car Group completes successful study of cordless charging for electric cars," Oct. 2013.

[4] M. Frazier, B. Taddese, T. Antonsen, and S. M. Anlage, "Nonlinear time reversal in a wave chaotic system," Physical review letters, vol. 110, no. 6, p. 063902, 2013.

[5] A. G. CEPNI, Experimental investigation of time-reversal techniques using electromagnetic waves. PhD thesis, Citeseer, 2005.

[6] K. Sherriff, "A dozen usb chargers in the lab: Apple is very good, but not quite the best," Oct 2012.

[7] W. R. Adey, "Biological effects of electromagnetic fields," Journal of cellular biochemistry, vol. 51, no. 4, pp. 410-416, 1993.

[8] ProCon, "20 highest and lowest radiation cell phones." Retrieved fro mthe CST website http://cellphones.procon.org/view.resource.php?resourceID=003054, aug 2015 . 
[9] R. Cheng, "Ikea to sell furniture that can wirelessly charge your phone." Retrieved from website: http://www.cnet.com/news/ikea-to-sell-furniture-thatcan-wirelessly-charge-your-phone/, feb 2015.

[10] M. Kesler, "Highly Resonant Wireless Power Transfer: Safe Efficient and over Distance," tech. rep., WiTricity, Watertown MA, 2013.

[11] C. Tucker, K. Warwick, and W. Holderbaum, "A contribution to the wireless transmission of power," International Journal of Electrical Power $\&$ Energy Systems, vol. 47, pp. 235-242, May 2013.

[12] K. Butler, "Tour WiTricity's Room of Tomorrow: Wireless Charging That's Flexible," Dec. 2013.

[13] E. Corporation, "Product overview." Retrieved from website: http://www.energous.com/product-overview/, aug 2016.

[14] J. Constine, "uBeam Declassifies Secrets To Try To Prove Wireless Power Is Possible," Nov. 2015.

[15] G. C. Audio, "Decibel(loudness) comparison chart." Retrieved from website: http://www.gcaudio.com/resources/howtos/loudness.html, aug 2007.

[16] I. Ossia, "Cota: Real wireless power," 2015.

[17] H. Zeine, "Wireless power transmission sytem," 2014. US Patent 8,854,176.

[18] Wi-Charge, "How it works." Retrieved from website: http://www.wicharge.com/technology.php?ID=25, mar 2016 . 
[19] M. Fink and others, "Time-reversed acoustics," Scientific American, vol. 281, no. 5, pp. 91-97, 1999.

[20] M. Fink, "Time-reversal waves and super resolution," Journal of Physics: Conference Series, vol. 124, no. 1, p. 012004, 2008.

[21] M. Fink and M. Tanter, "Multiwave imaging and super resolution," Phys. Today, vol. 63 , no. 2, pp. 28-33, 2010.

[22] P. Blomgren, G. Papanicolaou, and H. Zhao, "Super-resolution in time-reversal acoustics," The Journal of the Acoustical Society of America, vol. 111, no. 1, pp. $230-248,2002$.

[23] C. Prada, "The iterative time reversal mirror: A solution to self-focusing in the pulse echo mode," The Journal of the Acoustical Society of America, vol. 90, no. 2 , p. $1119,1991$.

[24] C. Prada, S. Manneville, D. Spoliansky, and M. Fink, "Decomposition of the time reversal operator: Detection and selective focusing on two scatterers," The Journal of the Acoustical Society of America, vol. 99, p. 2067, 1996.

[25] D. H. Chambers, "Target characterization using time-reversal symmetry of wave propagation," International Journal of Modern Physics B, vol. 21, pp. 3511-3555, Aug. 2007.

[26] D.-Q. Nguyen and W.-S. Gan, "The DORT solution in acoustic inverse scattering problem of a small elastic scatterer," Ultrasonics, vol. 50, pp. 829-840, Aug. 2010. 
[27] E. Barbieri and M. Meo, "Time reversal DORT method applied to nonlinear elastic wave scattering," Wave Motion, vol. 47, pp. 452-467, Nov. 2010.

[28] B. Wang, Y. Wu, F. Han, Y.-H. Yang, and K. J. R. Liu, "Green Wireless Communications: A Time-Reversal Paradigm," IEEE Journal on Selected Areas in Communications, vol. 29, pp. 1698-1710, Sept. 2011.

[29] B. T. Taddese, T. M. Antonsen, E. Ott, and S. M. Anlage, "Sensing small changes in a wave chaotic scattering system," Journal of Applied Physics, vol. 108, no. 11, p. 114911, 2010.

[30] Witricity, "Hrwpt in subsea application," 2013.

[31] G. Lerosey, J. De Rosny, A. Tourin, and M. Fink, "Focusing beyond the diffraction limit with far-field time reversal," Science, vol. 315, no. 5815, pp. 1120-1122, 2007.

[32] S. K. Hong, V. M. Mendez, T. Koch, W. S. Wall, and S. M. Anlage, "Nonlinear Electromagnetic Time Reversal in an Open Semireverberant System," Physical Review Applied, vol. 2, Oct. 2014.

[33] C. Torres-Torres, M. Trejo-Valdez, H. Sobral, P. Santiago-Jacinto, and J. ReyesEsqueda, "Stimulated emission and optical third-order nonlinearity in li-doped zno nanorods," The Journal of Physical Chemistry C, vol. 113, no. 31, pp. 1351513521, 2009.

[34] W. F. Smith, Waves and oscillations: a prelude to quantum mechanics. New York: Oxford University Press, 2010. 
[35] Griffiths, David, Introduction to Electrodynamics. Prentice Hall, 3 ed., 1999.

[36] C. S. Technology, "The finite integration technique." Retrieved fro mthe CST website https://www.cst.com/Products/CSTmws/FIT.

[37] J. B. Schneider, "Understanding the finite-difference time-domain method," School of electrical engineering and computer science Washington State University.-URL: http://www. Eecs. Wsu. Edu/ ${ }^{\sim}$ schneidj/ufdtd/(request data: 29.11. 2012), 2010.

[38] M. C. T. Weiland, "Discrete electromagnetism with the finite integration technique," Progress In Electromagnetics Research, vol. 32, pp. 65-87, 2001.

[39] D. H. Simpson, C. T. Chin, and P. N. Burns, "Pulse inversion Doppler: a new method for detecting nonlinear echoes from microbubble contrast agents," $\mathrm{Ul}$ trasonics, Ferroelectrics, and Frequency Control, IEEE Transactions on, vol. 46, no. 2, pp. 372-382, 1999.

[40] M. Frazier, B. Taddese, B. Xiao, T. Antonsen, E. Ott, and S. M. Anlage, "Nonlinear time reversal of classical waves: Experiment and model," Physical Review E, vol. 88, no. 6, p. 062910, 2013.

[41] S. Hemmady, X. Zheng, J. Hart, T. M. Antonsen Jr, E. Ott, and S. M. Anlage, "Universal properties of two-port scattering, impedance, and admittance matrices of wave-chaotic systems," Physical Review E, vol. 74, no. 3, p. 036213, 2006. 
[42] APD Semiconductor, AN-1012: Reverse Recovery Time (TRR) of the Super Barrier Rectifier, 2006.

[43] S. Davis, "Schottky diodes: the old ones are good, the new ones are better," Power Electronics, 2011.

[44] Macom, GaAs Flip Chip Schottky Barrier Diodes. Rev. V8.

[45] Y.-H. Suh and K. Chang, "A high-efficiency dual-frequency rectenna for 2.45-and 5.8-ghz wireless power transmission," Microwave Theory and Techniques, IEEE Transactions on, vol. 50, no. 7, pp. 1784-1789, 2002.

[46] A. Boaventura, D. Belo, R. Fernandes, A. Collado, A. Georgiadis, and N. Borges Carvalho, "Boosting the efficiency: Unconventional waveform design for efficient wireless power transfer," Microwave Magazine, IEEE, vol. 16, no. 3, pp. 87-96, 2015.

[47] Keysight Technologies, Infiniium 90000 Series Oscilloscopes. Rev. V8.

[48] Class-E Amplifier and Rectifier for Wireless Link with Secure Signal and Simulataneous Power Transmission Symposium on the Theory of Computing, no. 4, (Aveiro), WPTC, May 2016.

[49] A Novel Multi-coil Magnetically-Coupled Resonance Array for Wireless Power Transfer System Symposium on the Theory of Computing, no. 4, (Aveiro), WPTC, May 2016. 
[50] M. Fink, "Time-reversal acoustics in complex environments," Geophysics, vol. 71, no. 4, pp. SI151-SI164, 2006.

[51] B. T. Taddese, "Sensing small changes in a wave chaotic scattering system and enhancing wave focusing using time reversal mirrors," 2012.

[52] R. Wilson, "Propagation losses through common building materials 2.4 ghz vs 5 ghz. reflection and transmission losses through common building materials," Magis Networks, Inc.: San Diego, CA, USA, 2002. 